\title{
Effects of phosphatidylserine supplementation on exercising humans.
}

\author{
Kingsley, Michael lan Charles
}

How to cite:

Kingsley, Michael lan Charles (2006) Effects of phosphatidylserine supplementation on exercising humans.. thesis, Swansea University.

http://cronfa.swan.ac.uk/Record/cronfa42268

Use policy:

This item is brought to you by Swansea University. Any person downloading material is agreeing to abide by the terms of the repository licence: copies of full text items may be used or reproduced in any format or medium, without prior permission for personal research or study, educational or non-commercial purposes only. The copyright for any work remains with the original author unless otherwise specified. The full-text must not be sold in any format or medium without the formal permission of the copyright holder. Permission for multiple reproductions should be obtained from the original author.

Authors are personally responsible for adhering to copyright and publisher restrictions when uploading content to the repository.

Please link to the metadata record in the Swansea University repository, Cronfa (link given in the citation reference above.)

http://www.swansea.ac.uk/library/researchsupport/ris-support/ 


\section{Department of Sports Science}

University of Wales Swansea

\section{Effects of Phosphatidylserine \\ Supplementation on Exercising Humans}

Michael lan Charles Kingsley

Doctor of Philosophy

July 2006 
ProQuest Number: 10797976

All rights reserved

INFORMATION TO ALL USERS

The quality of this reproduction is dependent upon the quality of the copy submitted.

In the unlikely event that the author did not send a complete manuscript and there are missing pages, these will be noted. Also, if material had to be removed, a note will indicate the deletion.

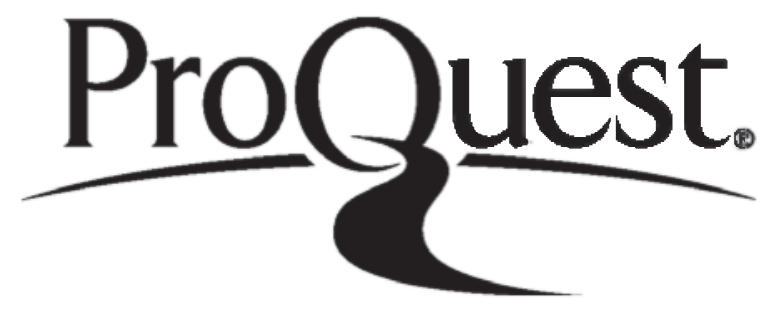

ProQuest 10797976

Published by ProQuest LLC (2018). Copyright of the Dissertation is held by the Author.

All rights reserved.

This work is protected against unauthorized copying under Title 17, United States Code Microform Edition @ ProQuest LLC.

ProQuest LLC.

789 East Eisenhower Parkway

P.O. Box 1346

Ann Arbor, Ml $48106-1346$ 


\section{DECLARATION}

I hereby declare that this thesis has been composed by myself, that the work is the result of my own investigations except where assistance has been otherwise acknowledged, that the work has not been previously submitted in candidature for any other degree, that all sources of information have been specifically acknowledged by means of reference, and that consent is provided for the thesis to be made available for photocopying and for inter-library loan.

Some of the findings presented in this thesis have been published in peer-reviewed journals, as follows:

Kingsley, M. (In press). Effects of phosphatidylserine on exercising humans. Sports Medicine.

Kingsley, M., Wadsworth, D., Kilduff, L.P., McEneny, J. and Benton, D. (2005). Effects of phosphatidylserine on oxidative stress following intermittent running. Medicine and Science in Sports and Exercise. 37 1300-1306.

Kingsley, M., Kilduff, L.P., McEneny, J., Dietzig, R.E. and Benton, D. (In press). Phosphatidylserine supplementation and recovery following downhill running. Medicine and Science in Sports and Exercise.

Kingsley, M., Miller, M., Kilduff, L.P., McEneny, J. and Benton, D. (2006). Effects of phosphatidylserine on exercise capacity during cycling in active males. Medicine and Science in Sports and Exercise. 38 64-71.

Signature: Date: $17-07-06$ 


\section{TABLE OF CONTENT}

\section{Page}

$\begin{array}{ll}\text { Declaration } & \text { i }\end{array}$

$\begin{array}{ll}\text { Contents } & \text { ii }\end{array}$

Acknowledgements

Summary $\quad$ vi

Table of abbreviation $\quad \mathrm{x}$

List of figures $\quad$ xii

List of tables $\quad$ xvi

Chapter 1: General Introduction $\quad 1$

1.1 Introduction 2

1.2 Phosphatidylserine supplementation 5

1.3 Mechanisms of phsophatidylserine action $\quad 11$

1.4 Effects of phosphatidylserine supplementation on 18 exercising humans

1.5 Effects of exercise on phosphatidylserine content within 22 cell membranes

$\begin{array}{ll}1.6 \text { Research aims } & 24\end{array}$

$\begin{array}{ll}\text { Chapter 2: } & \text { General Methods }\end{array}$

2.1 Subjects and study approval 28

2.2 Experimental design $\quad 29$

$\begin{array}{ll}2.3 \text { Supplementation } & 30\end{array}$

2.4 Dietary analysis 33

2.5 Anthropometric analysis 33

2.6 Experimental controls $\quad 34$

2.7 Blood analyses 34 
Chapter 3: Study one: Effects of phosphatidylserine supplementation on Performance and oxidative stress following intermittent running

3.1 Introduction 49

3.2 Methods $\quad 52$

$\begin{array}{ll}3.3 \text { Results } & 60\end{array}$

$\begin{array}{ll}3.4 \text { Discussion } & 74\end{array}$

Chapter 4: Study two: Phosphatidylserine supplementation and recovery following downhill running

$\begin{array}{ll}4.1 \text { Introduction } & 80\end{array}$

4.2 Methods 83

4.3 Results 91

$\begin{array}{ll}4.4 \text { Discussion } & 107\end{array}$

Chapter 5: Study three: Effects of phosphatidylserine supplementation on exercise capacity during staged intermittent cycling

5.1 Introduction

113

5.2 Methods

116

$\begin{array}{ll}5.3 \text { Results } & 127\end{array}$

$\begin{array}{ll}5.4 \text { Discussion } & 139\end{array}$

Chapter 6: General Discussion . 145

$\begin{array}{ll}\text { General Conclusions } & 161\end{array}$

$\begin{array}{ll}\text { References } & 163\end{array}$

$\begin{array}{ll}\text { Appendices } & 178\end{array}$ 


\section{ACKNOWLEDGEMENTS}

I am forever indebted to my wife, Kate, and my two sons, Sean and Daniel, who have permitted me to commit the extraordinary amount of additional time that is required to complete a doctorate level qualification whilst undertaking a full time academic post. Without their understanding it would not have been possible to complete this thesis. In addition, I wish to thank our extended families for supporting our decision to reside in the United Kingdom and pursue this career opportunity.

I wish to thank my supervisor, Professor James Watkins, for his support throughout this research. He provided me with the opportunity to work in the Department of Sports Science and the chance to initiate and develop the Exercise Physiology Laboratory at UWS. Both of which have been pivotal in allowing me to complete this research. In addition, I am grateful to my colleagues within the department for providing a stimulating environment in which to undertake and disseminate research.

The chance meeting, and ensuing conversations, with Professor David Benton led me down this eventful and fruitful line of research in the field of phosphatidylserine supplementation. Also, I thank Dr Jane McEneny for her collaboration, especially in the measurement of oxidative stress. I wish to express gratitude to my students, especially Mr Mark Miller and Mr Daniel Wadsworth, who helped invaluably in the data collection. Furthermore, the technical assistance provided by our laboratory 
technician, Mrs Rebecca Dietzig, and the technicians who work in Queen's University Belfast are gratefully appreciated.

Finally, my sincere gratitude goes to all of the participants who volunteered during this research; without their commitment, blood, sweat and tears this research would not have been possible. 


\section{SUMMARY}

The aim of this research was to investigate the effects of oral soy-derived phosphatidylserine (S-PtdSer) supplementation on the physiological responses to exercise, exercise performance/capacity and recovery following exercise.

The purpose of the first study was to investigate the effects of $750 \mathrm{mg}$ S-PtdSer or a glucose polymer placebo, administered daily for $10 \mathrm{~d}$, on markers of oxidative stress, perceived soreness, and muscle damage initiated by intermittent exercise (designed to simulate soccer match play) immediately followed by an exhaustive run. After familiarisation, 16 male soccer players completed an exhaustive intermittent exercise protocol on two further occasions (T1 and T2) separated by approximately $14 \mathrm{~d}$. Ten days prior to T2 the subjects were assigned, in a doubleblind manner, to receive either S-PtdSer (PS) or a placebo (P). Exercise time to exhaustion, sprint performance, ratings of perceived exertion and heart rate were recorded throughout both main exercise trials. Venous blood samples were obtained at rest (Pre-exercise), 15-min following exercise (Post-exercise), 24 hours after exercise (Post-24 h) and 48 hours after exercise (Post-48 h). Pre-exercise and Postexercise concentrations of plasma $\gamma$-tocopherol were increased following supplementation in PS, although supplementation had no effect on plasma concentrations of other non-enzymatic antioxidants (vitamin $\mathrm{C}, \alpha$-tocopherol, retinol and $\beta$-carotene). Serum cortisol concentrations, perceived soreness, markers of muscle damage (creatine kinase and myoglobin) and lipid peroxidation (hydroperoxides and conjugated diene lag times) were elevated to an equal extent in PS and P following exhaustive exercise prior to and following supplementation. The 
changes in running times to exhaustion from $\mathrm{T} 1$ to $\mathrm{T} 2$ in PS and P were $4.2 \pm 0.7$ and $-3.7 \pm 4.2 \%$, respectively $(P=0.084)$. Supplementation with S-PtdSer was not effective in attenuating the cortisol response, perceived soreness, and markers of muscle damage and lipid peroxidation following exhaustive running; however, PS tended to improve running and sprint performance following supplementation while no differences were observed following supplementation in $\mathrm{P}$.

As S-PtdSer supplementation tended to affect performance in study one, the original hypotheses were difficult to evaluate. Therefore, the purpose of study two was to investigate the effects of $750 \mathrm{mg} \mathrm{S-PtdSer}$, administered daily for 7 days prior to a bout of eccentric exercise and for 2 days following exercise, on delayed onset of muscle soreness and markers of muscle damage, inflammation and oxidative stress that follow prolonged downhill running. Following preliminary testing and a familiarisation session, eight recreationally active males repeated an individualised downhill run at $-16.5 \%$ for $51.0 \pm 1.5 \mathrm{~min}$ at $8.7 \pm 0.3 \mathrm{~km} \cdot \mathrm{h}^{-1}$ on four occasions (trials 1-4). Trials 1 and 3 were pre-supplementation control trials. After trials 1 and 3 the subjects received, in a double-blind and crossover fashion, either S-PtdSer or a glucose polymer placebo. Trials 2 and 3 were separated by a 4-week 'washout' period. Venous blood, joint ranges of motion, ratings of perceived soreness and feeling states were assessed prior to exercise, after exercise and at 24 and $48 \mathrm{~h}$ following exercise during each trial. Downhill running led to elevations in perceived soreness $(P<0.05)$, plasma creatine kinase activities $(P<0.001)$, myoglobin concentrations $(P<0.001)$, serum interleukin-6 concentrations $(P<0.001)$ and lipid hydroperoxide concentrations $(P<0.01)$. However, supplementation did not significantly attenuate these responses. These results suggested that the current 
regime of S-PtdSer supplementation did not afford additional protection against delayed onset of muscle soreness and markers of muscle damage, inflammation and oxidative stress that follow prolonged downhill running.

Based on the potential ergogenic properties of S-PtdSer identified in study one, the purpose of study three was to investigate the effects of $750 \mathrm{mg}$ S-PtdSer, administered daily for 10 days, on exercise capacity, oxygen uptake kinetic response, neuroendocrine function and feeling states during exhaustive intermittent cycling. Following preliminary testing and a familiarisation trial, fourteen active males completed a staged intermittent exercise protocol on two further occasions (T1 and T2) separated by $16 \pm 1 \mathrm{~d}$. The protocol consisted of three 10-min stages of cycling at 45,55 and $65 \% \dot{\mathrm{VO}}_{2}$ max followed by a final bout at $85 \% \dot{\mathrm{VO}}_{2}$ max that was continued until exhaustion. Approximately 5 days after T1 the subjects were assigned, in a double-blind manner, to either S-PtdSer (PS) or placebo (P). Breathby-breath respiratory data and heart rate were continually recorded throughout the exercise protocol, and blood samples were obtained at rest (Pre-exercise), during the rest periods within the protocol (Post-55, Post-65), at the end of exercise (Post-85), 20 min after the completion of exercise (Post-exercise) and the day following exercise (Post-24 h). The main finding of this study was that supplementation had a significant effect on exercise time to exhaustion at $85 \% \dot{\mathrm{VO}}_{2} \max (P=0.005)$. The exercise time to exhaustion in PS increased following supplementation (7:51 $\pm 1: 36$ $\min : s$ to $9: 51 \pm 1: 42 \min : s, P=0.001)$, while $P$ remained unchanged $(8: 09 \pm 0: 54$ min:s to 8:02 $\pm 0: 54 \mathrm{~min}: \mathrm{s}, P=0.670$ ). Supplementation did not significantly affect oxygen kinetic mean response times ( $\mathrm{MRT}_{\text {on }}$ and $\mathrm{MRT}_{\text {off }}$ ), serum cortisol concentrations, substrate oxidation and feeling states during the trial. This is the first 
study to report an improvement in exercise capacity following S-PtdSer supplementation.

Together, the results from this research provide evidence that short-term supplementation with $750 \mathrm{mg} \cdot \mathrm{d}^{-1} \mathrm{~S}-\mathrm{PtdSer}$ :

1. does not influence concentrations of cortisol or adrenocorticosteroid hormone in the circulation in young active male subjects. These data suggest that the current supplementation regime does not affect exercise-induced changes in the hypothalamo-pituitary-adrenal (HPA) axis.

2. is ineffective in attenuating the deleterious effects of exercise on perceived soreness, muscle damage, inflammation and oxidative stress in young active male subjects following eccentric exercise with a relatively low metabolic demand.

3. improved exercise capacity following intermittent cycling and tended to improve performance during prolonged intermittent running. These findings suggest that S-PtdSer might possess potential ergogenic properties. However, the mechanism(s) responsible for these findings remain to be determined. 


\section{TABLE OF ABBREVIATIONS}

Abbreviation

$\mathrm{ACTH}$

ANOVA

ATP

BC-PtdSer

$\mathrm{BM}$

$\mathrm{Ca}^{2+}$

$\mathrm{CHO}$

CK

DOMS

EDTA

EFI

ELISA

$\mathrm{H}^{+}$

$\mathrm{H}_{2} \mathrm{O}_{2}$

$\mathrm{Hb}$

Hct

HPA

HPO

HR

i.v.

IL-6
Meaning

Adrenocorticotropic hormone

Analysis of variance

Adenosine triphosphate

Bovine cortex-derived phosphatidylserine

Body mass

Calcium ion

Carbohydrate

Creatine kinase

Delayed onset of muscle soreness

Ethylenediaminetetra-acid

Exercise-induced feeling inventories

Enzyme-linked immunosorbent assay

Hydrogen ion

Hydrogen peroxide

Haemoglobin

Haematocrit

Hypothalamo-pituitary-adrenal

Hydroperoxide

Heart rate

Intravenous

Interleukin-6 


\section{TABLE OF ABBREVIATIONS (cont.)}

\begin{tabular}{|c|c|}
\hline $\mathrm{K}^{+}$ & Potassium ion \\
\hline LDL & Low density lipoprotein \\
\hline Lyso-PtdSer & Lysophosphatidylserine \\
\hline $\mathrm{MRT}_{\text {off }}$ & Mean response time for the off kinetic component \\
\hline $\mathrm{MRT}_{\text {on }}$ & Mean response time for the on kinetic component \\
\hline MSFT & Multistage fitness test \\
\hline $\mathrm{Na}^{+}$ & Sodium ion \\
\hline PtdSer & Phosphatidylserine \\
\hline ROM & Range of motion \\
\hline RPE & Rate of perceived exertion \\
\hline SEM & Standard error of mean \\
\hline S-PtdSer & Soy-derived phosphatidylserine \\
\hline SR & Sarcoplasmic reticulum \\
\hline TNF- $\alpha$ & Tumor necrosis factor-alpha \\
\hline $\mathrm{VO}_{2}$ & Oxygen uptake \\
\hline $\mathrm{VO}_{2} \max$ & Maximum oxygen uptake \\
\hline
\end{tabular}




\section{LIST OF FIGURES}

Figure Title $\quad$ Page

2.1 Low density lipoprotein (LDL) oxidation, displaying the 45 identification of lag time and time to half maximum oxidation $\left(t_{1 / 2 \max }\right)$.

3.1 Schematic of the intermittent exercise protocol 56

3.2 Performance during the multistage fitness test (MSFT) 64 undertaken during Part B of each exercise trial. Individual data is presented as open shapes and filled shapes present mean group values. Trial 1: pre-supplementation; Trial 2: post-supplementation.

3.3 Sprinting speeds during Part A of the exercise protocol. Values represent mean \pm SEM (supplementation group $\mathrm{x}$ trial $\mathrm{x}$ time of sample, $P=0.143$; time of sample, $P=0.055$ ). PS: phosphatidylserine group; P: placebo group.

3.4 Serum cortisol concentrations. Values represent mean \pm SEM $(\mathrm{N}=8$; supplementation group $\mathrm{x}$ trial $\mathrm{x}$ time of sample, $P=0.437$; time of sample, $P<0.001)$. PS: phosphatidylserine group; P: placebo group.

3.5 Serum myoglobin concentrations (a) and creatine kinase activities (b). Values represent mean $\pm \operatorname{SEM}(\mathrm{N}=8$; supplementation group $\mathrm{x}$ trial $\mathrm{x}$ time of sample, $P=0.190$ and $\mathrm{P}=0.596$, respectively; time of sample, $P<0.001$ and $P<0.001$, respectively). PS: phosphatidylserine group; P: placebo group.

3.6 Serum hydroperoxide concentrations. Values represent mean $\quad 70$ $\pm \mathrm{SEM}(\mathrm{N}=8$; supplementation group $\mathrm{x}$ trial $\mathrm{x}$ time of sample, $P=0.500$; time of sample, $P=0.001)$. PS: phosphatidylserine group; P: placebo group. 


\section{LIST OF FIGURES (cont.)}

Figure Title

Page

3.7 Conjugated diene lag times. Values represent mean $\pm \mathrm{SEM}$

$(\mathrm{N}=8$; supplementation group $\mathrm{x}$ trial $\mathrm{x}$ time of sample, $P=0.489$; time of sample, $P=0.011)$. PS: phosphatidylserine group; P: placebo group.

4.1 Schematic diagram of the experimental design.

4.2 (a) Plasma adrenocorticotropic hormone (ACTH) concentrations and (b) plasma cortisol concentrations throughout each trial. Values represent mean $\pm \mathrm{SEM}(\mathrm{N}=8)$. Pre-P: trial prior to supplementation with placebo; P: trial supplemented with placebo; Pre-PS: trial prior to supplementation with phosphatidylserine; PS: trial supplemented with phosphatidylserine.

$\begin{array}{ll}4.3 & \text { (a) Plasma myoglobin concentrations and (b) plasma creatine } 95\end{array}$ kinase activities throughout each trial. Values represent mean \pm SEM $(\mathrm{N}=8)$. Pre-P: trial prior to supplementation with placebo; P: trial supplemented with placebo; Pre-PS: trial prior to supplementation with phosphatidylserine; PS: trial supplemented with phosphatidylserine.

4.4 Serum interleukin (IL-6) concentrations throughout each trial. 99 Values represent mean $\pm \mathrm{SEM}(\mathrm{N}=8)$. Pre-P: trial prior to supplementation with placebo; P: trial supplemented with placebo; Pre-PS: trial prior to supplementation with phosphatidylserine; PS: trial supplemented with phosphatidylserine. 


\section{LIST OF FIGURES (cont.)}

Figure Title

Page

4.5 Serum hydroperoxide concentrations throughout each trial.

100

Values represent mean $\pm \mathrm{SEM}(\mathrm{N}=8)$. Pre-P: trial prior to

supplementation with placebo; $\mathrm{P}$ : trial supplemented with

placebo; Pre-PS: trial prior to supplementation with

phosphatidylserine; PS: trial supplemented with

phosphatidylserine.

4.6 Change in conjugated diene concentrations throughout each

102

trial. Values represent mean $\pm \operatorname{SEM}(\mathrm{N}=8)$. Pre-P: trial prior to

supplementation with placebo; P: trial supplemented with

placebo; Pre-PS: trial prior to supplementation with

phosphatidylserine; PS: trial supplemented with

phosphatidylserine.

4.7 Time to half of maximum low density lipoprotein oxidation

103

$\left(\mathrm{T}_{1 / 2 \max } \mathrm{LDL}\right.$ oxidation) throughout each trial. Values

represent mean $\pm \operatorname{SEM}(\mathrm{N}=8)$. Pre-P: trial prior to

supplementation with placebo; P: trial supplemented with

placebo; Pre-PS: trial prior to supplementation with

phosphatidylserine; PS: trial supplemented with

phosphatidylserine.

5.1 Schematic diagram of the experimental design

5.2 Average oxygen uptake for one subject for the bouts of

moderate exercise. Schematic showing the phase II on-kinetic response and the off-kinetic response. 


\section{LIST OF FIGURES (cont.)}

$\begin{array}{lll}\text { Figure Title } & \text { Page }\end{array}$

5.3 Exercise times to exhaustion at $85 \% \dot{\mathrm{V}}_{2} \mathrm{O}_{\text {max }}$ completed at the end of each exercise trial. Individual data are presented as open shapes and mean data are presented as filled shapes. PS: phosphatidylserine group; P: placebo group. (supplementation group $\mathrm{x}$ trial interaction, $P=0.007$ ).

5.4 (a) Energy expenditure, (b) carbohydrate oxidation rates, and

(c) fat oxidation rates during each main exercise trial. Values represent mean \pm SEM $(\mathrm{N}=7)$. PS: phosphatidylserine group; P: placebo group. Trial 1: pre-supplementation; Trial 2: postsupplementation.

5.5 (a) On-kinetic mean response times and (b) off-kinetic mean response times during moderate and very heavy exercise stages. Values represent mean \pm SEM $(\mathrm{N}=7)$. PS: phosphatidylserine group; P: placebo group. Trial 1: presupplementation; Trial 2: post-supplementation.

5.6 Serum cortisol concentrations throughout each trial. Values

represent mean $\pm \operatorname{SEM}(\mathrm{N}=8)$. Pre-P: trial prior to supplementation with placebo; P: trial supplemented with placebo; Pre-PS: trial prior to supplementation with phosphatidylserine; PS: trial supplemented with phosphatidylserine. 


\section{LIST OF TABLES}

Table Title

Page

1.1 Phosphatidylserine supplementation studies with human participants that have undertaken exercise protocols.

2.1 Typical composition of concentrated phosphatidylserine . 31 product.

2.2 Approximate composition of final phosphatidylserine product. 32

3.1 Subject characteristics for phosphatidylserine supplementation 53 group (PS) and placebo group (P).

3.2 Estimated percentage changes in plasma volume throughout 61 both trials for phosphatidylserine supplementation group and placebo group.

3.3 Energy intake and dietary composition during both exercise 62 trials for phosphatidylserine supplementation group and placebo group.

3.4 Perceived ratings of general soreness (GS), quadriceps 68 soreness (QS), and hamstring soreness (HS) in all trials.

3.5 Plasma antioxidant vitamin concentrations throughout both $\quad 73$ trials for PS and P groups.

4.1 Blood lactate concentrations, blood glucose concentrations $\quad 92$ and estimated plasma volume changes during all trials.

4.2 Perceived ratings of general soreness (GenS) quadriceps 97 soreness (QS), hamstring soreness (HS), and gluteal soreness (GS) during all trials.

4.3 Active range of motion during knee flexion (knee ROM) and 98 hip flexion (hip ROM) during all trials.

4.4 Plasma antioxidant vitamin concentrations during all trials. 104 


\section{LIST OF TABLES (cont.)}

Table Title

4.5 Exercise-induced feeling inventory (EFI) subscale scores $\quad 106$ during all trials.

5.1 Subject characteristics for phosphatidylserine supplementation 117 group (PS) and Placebo group (P).

5.2 Last minute heart rate and oxygen uptake during both exercise 129 trials for phosphatidylserine supplementation group and placebo group.

5.3 Estimated percentage changes in plasma volume throughout both trials for phosphatidylserine supplementation group and placebo group.

5.4 Blood lactate and glucose concentrations during both exercise $\quad 135$ trials for phosphatidylserine supplementation group and placebo group.

5.5 Exercise-induced feeling inventory (EFI) subscale scores throughout both trials for phosphatidylserine group and placebo group. 
CHAPTER ONE

\section{GENERAL INTRODUCTION}




\subsection{INTRODUCTION}

Phosphatidylserine (PtdSer) was initially identified as a constituent of the cephalin fraction of bovine brain (Folch and Schneider, 1941). All phospholipids, which include the PtdSer species, are comprised of a glycerol molecule, two fatty acids, and a phosphate group. Although the head group is similar in all PtdSer species, the associated fatty acids can vary in composition (Blokland et al., 1999). Molecular PtdSer species derived from soybean (S-PtdSer) are rich in linoleic and palmitic acids whereas stearic and oleic acids are abundant in those derived from bovine cortex (BC-PtdSer) (Sakai et al., 1996). It has been suggested that this variation in chemical structure might alter the functionality of this acidic phospholipid within cell membranes. Nevertheless, similar pharmacological responses (Sakai et al., 1996) and behaviour effects (Sakai et al., 1996; Blokland et al., 1999) have been reported in rodents following PtdSer supplements derived from soybean and bovine cortex.

It is now evident that PtdSer is a universal component of the plasma membranes in eukaryotic cells (Vance and Steenbergen, 2005) and is integral in the maintenance of a wide range of normal cellular functions. Rich dietary sources of PtdSer include offal and fish (Souci et al., 2000). In addition, mammalian cells are capable of de novo PtdSer synthesis from phosphatidylcholine catalysed by the enzyme phosphatidylserine synthase 1 (Voelker and Frazier, 1986) and from phosphatidylethanolamine using the enzyme phosphatidylserine synthase 2 (Suzuki and Kanfer, 1985); consequently, PtdSer might not qualify as an 'essential' nutrient. 
The quantity of research that has been, and continues to be, undertaken on the biochemistry of PtdSer is a testament to the array of functions performed by this group of phospholipids. For the purpose of this thesis, these biochemical functions have been broadly defined as: (1) enzyme cofactors; (2) neuroendocrine actions; (3) actions on the immune system; and (4) potential antioxidant actions. A review of these functions can be found in Section 1.3.

It has been hypothesised that PtdSer influences cognitive function by maintaining the excitability of neuronal membrane (through the activation of various ATPases; Section 1.3), maintaining dendritic density (as a cofactor for protein kinase C and Raf-1 protein kinase; Section 1.3), and maintaining cell-to-cell communication (by moderating the release of various neurotransmitters, such as dopamine and acetylcholine; Section 1.3). Indeed, the administration of PtdSer to rodents enhances cognitive function (Blokland et al., 1999), improves learning/memory (Sakai et al., 1996; Furushiro et al., 1997; Alves et al., 2000; Suzuki et al., 2001) and attenuates the effects of stress (Drago et al., 1991). Additionally, the pharmacological actions of PtdSer on cognitive functions have been reported in other animals (Koutoku et al., 2005). Furthermore, exogenous PtdSer has been demonstrated to exert therapeutic actions on higher brain functions in human patients with cognitive decline (Amaducci and SMID group, 1988; Crook et al., 1992; Cenacchi et al., 1993), although the efficacy of PtdSer supplementation in the treatment of cognitive decline has been a matter of debate (Soares and Gershon, 1994).

The majority of investigations that have evaluated the effects of PtdSer supplementation have appraised this potential therapeutic agent in humans and 
animals with cognitive dysfunction (Section 1.2). In addition, PtdSer has been demonstrated to exert a range of pharmacological actions in healthy individuals (Section 1.2). The purpose of this chapter was to evaluate the potential application of PtdSer as a supplement for use by humans, with a particular focus on the pharmacological effects of PtdSer supplementation on the responses to exercise. 


\subsection{PHOSPHATIDYLSERINE SUPPLEMENTATION}

Many of the proposed pharmacological effects following PtdSer administration rely on exogenous PtdSer reaching the inner membrane and exerting the various biochemical actions that have been ascribed to endogenous PtdSer (Pepeu et al., 1996). In addition, it is plausible that unbound PtdSer (i.e., PtdSer that is not incorporated in cell membranes) might be effective also as an antioxidant and, subsequently, contribute to the cellular defence against oxidative stress.

\section{Fate of Exogenous Phosphatidylserine}

In-vitro studies have established that exogenous PtdSer is effectively internalised and incorporated in cultured mammalian cells (Kuge et al., 1986; Nishijima et al., 1986; Voelker and Frazier, 1986). Consequently, PtdSer supplementation regimes that are successful in elevating extracellular PtdSer will probably enhance intracellular PtdSer. In support of this hypothesis, Palatini et al. (1991) demonstrated that radioactively labelled PtdSer was present in organ tissues as well as in the plasma of rats $60 \mathrm{~min}$ following the bolus intravenous (i.v.) injection of radioactively labelled PtdSer liposomes. These authors rationalised that the rapid initial decline in plasma concentrations (half-life of $0.85 \mathrm{~min}$ ) was caused by uptake in the mononuclear phagocyte system, as demonstrated by the accumulation of PtdSer in liver and spleen tissues. The slow component (half-life of $40 \mathrm{~min}$ ) was hypothesised to reflect PtdSer that is incorporated in high density plasma lipoproteins. In addition, the analysis of biotransformation products suggested that 
decarboxylation to phosphatidylethanolamine and extensive hydrolytic degradation probably reflected the mechanisms of PtdSer uptake, incorporation into the plasma membrane and internalisation by endocytosis, respectively (Palatini et al., 1991). Although not specifically addressed in this study, it is likely that the majority of the PtdSer that interacts with the phospholipid pool of the plasma membrane will be maintained in the inner leaflet of the plasma membrane while a small proportion will be translocated to the mitochondria and golgi apparatus (Heikinheimo and Somerharju, 2002).

Bruni et al. (1992) investigated the fate of PtdSer following orally administered PtdSer using duodenal infusion with radioactively labelled PtdSer in rats. Following the determination of radioactivity in the lymph over $5 \mathrm{~h}$, these authors concluded that the majority of the PtdSer supplement was hydrolysed or converted to other phospholipids (mainly phosphatidylethanolamine and phosphatidylcholine); importantly, small quantities of PtdSer reached the systemic circulation. Consequently, oral supplementation potentially increases the systemic PtdSer pool and probably increases tissue PtdSer.

In summary, the fate and pharmacokinetics of PtdSer in humans, following oral or i.v. PtdSer supplementation, have yet to be characterised. However, the data from invitro mammalian cell cultures and in-vivo rat experiments provide indirect evidence that i.v. and oral administration of PtdSer are effective in increasing extracellular PtdSer and lead to quantifiable, albeit small, increases in PtdSer within the plasma membrane of tissues. 
The biochemical tolerability of orally administered bovine cortex derived PtdSer (BC-PtdSer; $300 \mathrm{mg} \cdot \mathrm{d}^{-1}$ for $30 \mathrm{~d}$ ) was determined in 130 human patients (Cenacchi et al., 1987). Using laboratory data from 6 different laboratories these authors calculated changes (pre to post supplementation) in a range of haematological variables. PtdSer caused no detrimental changes in blood biochemistry; indeed, favourable non-significant reductions in uric acid and serum glutamate pyruvate transaminase were identified following supplementation. However, BC-PtdSer is currently considered unsuitable for use in human supplementation due to the potential transfer of infectious disease, such as bovine spongiform encephalopathy (mad cow disease), through the consumption of prion contaminated brain.

S-PtdSer has been proposed as an alternative supplement. Jorissen et al. (2002) reported the effects of placebo, 300 or $600 \mathrm{mg}^{-1} \mathrm{~d}^{-1}$ of S-PtdSer, administered orally to 120 elderly patients, on biochemical and haematological safety parameters, blood pressure, heart rate and adverse effects at baseline and after 12 weeks of treatment in a randomised placebo-controlled clinical trial. These authors reported no differences between treatment groups in all the outcomes measured and, therefore, concluded that S-PtdSer was a safe nutritional supplement when administered under these conditions. Additionally, no side effects have been reported when young healthy adults consume up to $800 \mathrm{mg} \cdot \mathrm{d}^{-1}$ of BC-PtdSer (Monteleone et al., 1992) or SPtdSer (Fahey and Pearl, 1998) for 10 and 12 d, respectively. Moreover, according to Pepeu et al. (1996), no side effects had been reported in the very large number of patients that have been treated with PtdSer prior to the publication of their review 
and this statement appears to remain current. Indeed all fatty acids, including PtdSer, are considered non-toxic to individuals without specific allergies, at least in the quantities likely to be obtained from diet and other supplements.

In summary, PtdSer was initially obtained from bovine cortex; however, due to the potential transfer of infectious disease, this supplement is now considered unsuitable for human consumption. More recently, soy-derived PtdSer (S-PtdSer) has become accepted as a safe alternative with no known side effects.

\section{Supplementation Strategies}

Monteleone et al. (1990) demonstrated that the acute i.v. administration of 50 and 75 mg BC-PtdSer blunted plasma adrenocorticotropic hormone (ACTH) and cortisol responses to cycling in 8 healthy untrained males. Although there was a tendency for lower concentrations of these hormones during exercise following $75 \mathrm{mg} \mathrm{BC}-\mathrm{PtdSer}$ when compared with $50 \mathrm{mg}$ BC-PtdSer, these responses were not significantly different. Consequently, there was no evidence to substantiate a dose-response to this attenuation in the neuroendocrine response to cycling.

More recently, oral supplementation has been the method of choice for supplying exogenous PtdSer to humans. The oral administration of $800 \mathrm{mg} \cdot \mathrm{d}^{-1} \mathrm{BC}-\mathrm{PtdSer}$ for $10 \mathrm{~d}$ was demonstrated to significantly attenuate plasma cortisol concentrations in healthy inactive males (Monteleone et al., 1992). However, $400 \mathrm{mg} \cdot \mathrm{d}^{-1}$ BC-PtdSer did not significantly affect cortisol concentrations (Monteleone et al., 1992); 
thereby, suggesting that, over a 10-d supplementation period, higher doses might be necessary to counteract the stress-induced activation of the hypothalamo-pituitaryadrenal (HPA) axis in humans. However, the optimal dose of S-PtdSer might vary between participants.

Most studies that have reported enhanced higher brain functioning following PtdSer supplementation have employed lower daily doses $\left(<500 \mathrm{mg} \cdot \mathrm{d}^{-1}\right)$ for longer durations. For example, Cenacchi et al. (1993) reported improvements in behavioural and cognitive functions in a group of geriatric patients with cognitive impairment after $300 \mathrm{mg} \cdot \mathrm{d}^{-1} \mathrm{BC}-\mathrm{PtdSer}$ for $6 \mathrm{months}$. Furthermore, $300 \mathrm{mg} \cdot \mathrm{d}^{-1} \mathrm{~S}$ PtdSer for 1 month has been shown to be associated with feeling less stressed and having better mood in a sub-group of healthy young males (Benton et al., 2001). Alternatively, Fahey and Pearl (1998) confirmed the potential efficacy of $800 \mathrm{mg} \cdot \mathrm{d}^{-1}$ S-PtdSer over 2 weeks using a counter-balanced, placebo-controlled, cross-over study design in 11 healthy resistance trained males. These authors reported that this supplementation regime was effective in reducing perceived muscle soreness and increasing subjective feelings of well-being. Although, at present, too few data exists to determine the optimal S-PtdSer supplementation regime (to include: dose, length of supplementation duration and timing of supplementation), oral doses of up to $800 \mathrm{mg} \cdot \mathrm{d}^{-1} \mathrm{~S}$-PtdSer for durations of up to 2 weeks have been demonstrated to modify physiological and higher brain functions while no detrimental side effects have been reported. Furthermore, it remains unclear whether the optimal supplementation regime will vary depending upon individual characteristics such as age, gender or training status. 
The pharmacokinetics of PtdSer following supplementation in humans has not been elucidated and the turnover of PtdSer in the cell membrane is unknown; consequently, no information is currently available on the washout duration of exogenous PtdSer. In addition, evidence has been presented to demonstrate that the actions of PtdSer continue after supplementation has ceased (Amaducci and SMID group, 1988), suggesting that the incorporated PtdSer might accumulate in body tissues. Therefore, the interpretation of studies that have employed a simple crossover experimental design might be problematic.

In summary, investigators have administered PtdSer through i.v. and oral routes; nevertheless, oral supplementation has greater appeal. Although the optimal oral dose of PtdSer is not clear at present, doses of $800 \mathrm{mg} \cdot \mathrm{d}^{-1}$ for up to 2 weeks have been demonstrated to attenuate the exercise-induced activation of the HPA axis, reduce perceived muscle soreness and improve well-being. 


\subsection{MECHANISMS OF PHOSPHATIDYLSERINE ACTION}

In addition to the structural role of PtdSer, this phospholipid species is required for a range of specific cellular functions. For the purpose of this chapter these functions have been broadly defined as: (1) enzyme cofactors; (2) neuroendocrine actions; (3) actions on the immune system; and (4) potential antioxidant actions.

\section{Enzyme Cofactor}

A myriad of proteins have been identified that require PtdSer for optimal activity (for a review consult Toffano and Bruni (1980)); of which, a number might have specific relevance to physiological function during exercise. Phosphatidylserine is the most effective phospholipid in activating the conventional isoforms of protein kinase C (Takai et al., 1979), which undertake crucial functions in diverse signal transduction pathways (Nishijima et al., 1986). Similarly, PtdSer interacts with Raf1 protein kinase to promote a cascade of reactions that are believed to be crucial during cell growth (Ghosh et al., 1994), through the translocation of protein to the plasma membrane (Nagai et al., 1999). Importantly, enrichment of rat neuronal cells with PtdSer has been demonstrated to reduce Raf-1 translocation and ultimately reduce apoptosis induced by serum starvation (Kim et al., 2000). These data suggest that increasing the cell membrane concentration of PtdSer might afford protection against cell death, at least in neuronal cells. 
In-vitro studies have demonstrated that low concentrations of PtdSer are effective in activating $\left(\mathrm{Na}^{+}-\mathrm{K}^{+}\right)$-dependent ATPase in mammalian kidney (Specht and Robinson, 1973 ) and brain (Tsakiris and Deliconstantinos, 1984) preparations. Similarly, $\mathrm{Ca}^{2+}$ ATPase, an enzyme primarily responsible for $\mathrm{Ca}^{2+}$ re-uptake from the muscle cytosol into the sarcoplasmic reticulum (SR), is known to require PtdSer (Morrot et al., 1990; Sepulveda and Mata, 2004). Furthermore, PtdSer is preferentially associated with the $\mathrm{Ca}^{2+}$-ATPase of cardiac and skeletal muscle $\mathrm{SR}$, at least in isolated canine tissues (Bick et al., 1998).

The aetiology of fatigue, defined as the decline in muscle performance during exercise, has not been fully elucidated and might include central and peripheral components (Kent-Braun, 1999). As the exercise (mode, intensity and duration) and participant characteristics (such as muscle characteristics and training status) probably influence the site of fatigue, many theories currently exist to explain fatigue. Nevertheless, failure in the excitation-contraction coupling process is currently considered to be a major mechanism responsible for peripheral fatigue (Allen, 2004). This process includes action potential propagation along the sarcolemma, $\mathrm{Ca}^{2+}$ release from the $\mathrm{SR}$ leading to activation of myofilament contraction, and $\mathrm{Ca}^{2+}$ reuptake into the SR (Giannesini et al., 2003). Therefore, it is plausible that exogenous PtdSer might maintain ionic balance for longer during exercise by enhancing enzyme activities and consequently delay the onset of fatigue. 


\section{Neuroendocrinological Regulation}

Seminal work by Bruni et al. (1976) confirmed that PtdSer was able to modify endocrine function. These authors showed that injecting mice with PtdSer liposomes promoted increases in brain glucose and blood glucose that were probably associated with the release of catecholamines (Toffano et al., 1976) and histamine from peripheral tissues (Bigon et al., 1979). Since this time PtdSer has been demonstrated to influence cortical acetylcholine release (Casamenti et al., 1979; Pepeu et al., 1986; Casamenti et al., 1991; Yamatoya et al., 2000), dopamine and noradrenaline release (Toffano et al., 1976), at least following i.v. administration in rodents.

Monteleone and colleagues were among the first to identify the effectiveness of BCPtdSer to influence neuroendocrine functions in humans. Both i.v. (Monteleone et al., 1990) and oral (Monteleone et al., 1992) supplementation methods have been shown to attenuate the cortisol and ACTH responses to acute cycling exercise.

It has been proposed that changes in neuroendocrine function might affect cognitive functions and feelings. Benton et al. (2001) confirmed that S-PtdSer supplementation $\left(300 \mathrm{mg} \cdot \mathrm{d}^{-1}\right.$ continued for $30 \mathrm{~d}$ ) led to improvements in the feeling states of a sub-group of young healthy participants when they were asked to perform demanding mental tasks. However, prior to the current research, no data existed to assess the effects of PtdSer supplementation on feeling states during exercise. 


\section{Effects on Immune Function}

The acute inflammatory response is the body's generic reply to a variety of stimuli that initiate the damage of tissues. This non-specific response mobilises the defences that lead to the destruction and removal of injured tissues and is characterised by local dilation of blood vessels, heat, swelling, pain and disturbances of function. Following injury, including exercise induced tissue damage, mediators of inflammation such as plasma kinins and histamine derived from mast cells and basophils are activated in order to increase the permeability of blood vessels. In addition, circulatory increases in proinflammatory and inflammation-responsive cytokines, such as tumor necrosis factor-alpha (TNF- $\alpha$ ), interleukin-1 (IL-1) beta and interleukin-6 (IL-6), are observed. Neutrophils and macrophages migrate from blood to the site of tissue damage and phagocytose damaged cells.

The ability of PtdSer to regulate immune function in mammalian tissues was recognised in the early 1970's (Goth et al., 1971; Mongar and Svec, 1972). Mongar and Svec (1972) demonstrated, using in-vitro methods, that low concentrations of PtdSer augmented the release of histamine by antigen from sensitized rat tissues. Subsequently, PtdSer has been demonstrated to enhance the calcium dependent anaphylactic histamine release initiated by $\operatorname{IgE}$ antibodies (Shores and Mongar, 1980) and IgG antibodies (Moodley and Mongar, 1981). Furthermore, i.v. injection with lysophosphatidylserine (lyso-PtdSer), the deacylated product of PtdSer hydrolysis that is produced at the site of tissue injury, has been shown to increase blood histamine concentrations in mice by specifically activating connective tissue mast cells (Monastra et al., 1991). However, it remains plausible that the in-vitro 
activation of mast cells might be peculiar to the administration of PtdSer via injections, where transient increases in plasma PtdSer and lyso-PtdSer (especially at the site of injection) have been reported (Palatini et al., 1991).

As PtdSer is usually maintained in the inner bilayer of the plasma membrane (Devaux, 1991), contact with the extracellular environment is normally prevented; however, when cells become damaged PtdSer becomes externalised to the outer bilayer of the plasma membrane. Externalisation of PtdSer is believed to signal apoptosis and initiate cell phagocytosis (Tanaka and Schroit, 1983; Tyurina et al., 2000). Phagocytes rapidly identify, engulf and remove apoptotic cells; thus, preventing the release of potentially toxic intracellular contents from damaged cells.

Additionally, PtdSer exposure inhibits the production of pro-inflammatory cytokines (Gilbreath et al., 1986; Aramaki et al., 1997) and induces anti-inflammatory responses (Huynh et al., 2002) in in-vitro preparations. Based on in-vitro tests demonstrating that PtdSer does not affect antigen processing and presentation by macrophages, these authors suggested that these immunological actions reflect a direct action of PtdSer with the lymphocytes. In addition, the modulation of immune response was evident when PtdSer was administered $4 \mathrm{~h}$ prior to immunisation; however, PtdSer had no effect when administered $24 \mathrm{~h}$ prior to or indeed following immunisation. These findings suggest that the influence of PtdSer on lymphocyte activity is transient (Ponzin et al., 1989). Consequently, the actions of PtdSer moderate the acute inflammatory response, thereby reducing non-specific damage to normal cells in the local environment. 
Furthermore, in-vivo experiments have demonstrated anti-inflammatory properties of PtdSer in a variety of animal models. The i.v. administration of BC-PtdSer has been demonstrated to reduce $\mathrm{T}$-dependent and $\mathrm{T}$-independent antibody production to immunisation in mice (Ponzin et al., 1989). Also, the injection of mice (intraperitoneal injection $30 \mathrm{mg} \cdot \mathrm{d}^{-1}$ for $3 \mathrm{~d}$ ) and rabbits (i.v. injection $30 \mathrm{mg} \cdot \mathrm{d}^{-1}$ for 5 d) with phospholipid mixtures rich in BC-PtdSer effectively reduced the endotoxininduced production of TNF- $\alpha$ (Monastra and Bruni, 1992). In addition, the introduction of a synthetic analogue of PtdSer (cholesterylphosphorylserine) (Bruni et al., 1994) via intraperitoneal injection has been shown to inhibit the rise in plasma TNF- $\alpha$ that followed monocyte activation in rodents (Facci et al., 2000). Acute inflammation in rats, initiated by subcutaneous toxin injection, has been demonstrated to increase endogenous PtdSer synthesis in the endoplasmic reticulum of liver tissue (Vincent et al., 2001). At present the significance of this increase in PtdSer is unclear; however, it might reflect an increased requirement for PtdSer during the acute inflammation response.

Although the mechanism(s) by which PtdSer inhibits the immune response remains unclear, recent data suggest that PtdSer inhibits immune responses by acting on cell types (e.g., macrophages, fibroblasts, neutrophils, endothelial cells, epithelial cells) at the site of inflammation (Hoffmann et al., 2005). However, the efficacy of PtdSer as an immunoactive agent in humans remains, in the main, unscrutinised. Consequently, investigations are warranted to assess the effects of S-PtdSer supplementation on markers of inflammation following exercise. 


\section{Potential Antioxidant}

PtdSer has been demonstrated, using in-vitro experiments, to possess an ironbinding ability that is similar to other acid phospholipids (such as phosphatidic acid, phosphatidylglycerol) (Dacaranhe and Terao, 2001). In addition to this function, Dacaranhe and Terao (2001) demonstrated that PtdSer was unique in its ability to suppress the iron-induced lipid peroxidation of egg yolk phosphatidylcholine. BCPtdSer has been shown to reduce human low density lipoprotein (LDL) oxidation mediated by cupric sulphate (Lou et al., 1994), as measured by the formation of liperoxides and conjugated dienes. However, these authors also reported that BCPtdSer did not inhibit the oxidation of human LDL mediated by macrophages in the presence of a complex cell medium (Ham's F-10). Even so, the incubation of human skin cells with BC-PtdSer has been previously shown to attenuate lactate dehydrogenase production in response to generated oxygen free radicals using invitro preparations (Latorraca et al., 1993). The processes responsible for tissue oxidation have not been fully defined and as such the specific mechanism(s) responsible for the reported antioxidant properties of PtdSer, additional to the binding of free metal ions, are not clear and might explain these equivocal findings. Nevertheless, these studies suggest that under a variety of circumstances, PtdSer might be an effective antioxidant against cell and tissue oxidation. 


\subsection{EFFECTS OF PHOSPHATIDYLSERINE SUPPLEMENTATION ON EXERCISING HUMANS}

PubMed and Science Direct were used as the primary databases for searching the topic of PtdSer supplementation and exercise. Prior to the current research, three peer-reviewed full articles were identified from this search that involved human subjects. A summary of these studies is presented in Table 1.1. One study was identified that used PtdSer derived from bovine cortex while the remaining studies used PtdSer derived from soy beans (Table 1.1). As described previously, in the introduction section of this chapter, the structure of PtdSer is dependent upon the source used to obtain the supplement; consequently, the source used to obtain concentrated PtdSer for oral supplements might be important when comparing the outcomes from these studies.

\section{Effects on Neuroendocrinological Function}

As previously mentioned, orally administered BC-PtdSer has been reported to blunt the cortisol response to acute exercise stress in untrained males (Monteleone et al., 1992). These authors interpreted this change to represent an attenuation in the stressinduced activation of the HPA axis (Monteleone et al., 1990). However, training status of the participants might affect this outcome, especially considering that trained subjects experience attenuated HPA alterations during exercise (Luger et al., 1987). Prior to the current research, no data existed that investigates the effects of PtdSer supplementation on neuroendocrine responses to exercise in active participants. 


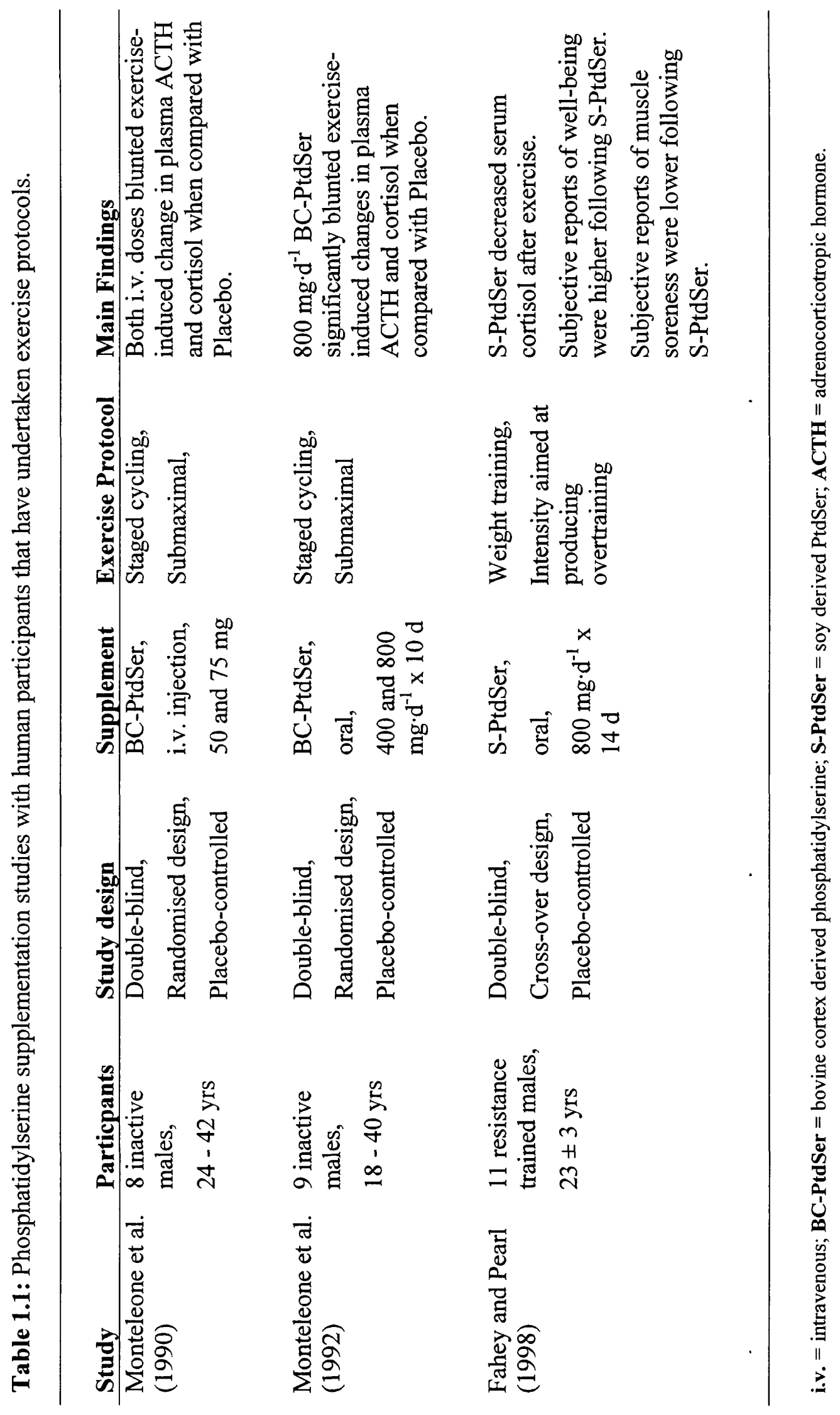




\section{Effects on Subjective Feelings}

Short-term S-PtdSer supplementation has been reported to improve subjective wellbeing when compared to supplementation with placebo during two weeks of heavy resistance training that was designed to cause overtraining (Fahey and Pearl, 1998). Furthermore, subjective feelings of soreness were purportedly lower following supplementation with S-PtdSer when compared to supplementation with the placebo. Although the potential mechanism(s) responsible for these findings were speculative, these authors suggested that alterations in the HPA axis during exercise and during the ensuing recovery periods might have led to reductions in circulatory $\mathrm{ACTH}$ and cortisol concentrations which might have attenuated the perceptions of well-being and muscle soreness.

\section{Effects on Exercise-Induced Muscle Damage and Delayed Onset of Muscle}

\section{Soreness}

As a ubiquitous component of the cell membrane, PtdSer is thought to play an important function in maintaining the structural integrity of cell membranes (Singer and Nicolson, 1972). Consequently, the effective incorporation of exogenous PtdSer within the cell membrane might alter cell membrane fluidity and reduce the physical damage that is associated with unaccustomed muscle activity. In addition, following exercise, secondary sources of muscle damage include: (1) invading phagocytes during the acute inflammatory response that follows initial muscle damage (Tidball, 2005); (2) loss of $\mathrm{Ca}^{2+}$ homeostasis (Armstrong et al., 1991); and (3) elevated 
oxidative stress from increases in oxygen flux through the mitochondria (Dekkers et al., 1996). Therefore, it is plausible that the antioxidant and immunosuppressive properties of PtdSer might afford additional protection against secondary muscle damage.

Structural damage to muscle cells is associated with leakage of proteins, which are usually maintained in the intracellular compartment, such as creatine kinase (CK) and myoglobin into the circulation (Feasson et al., 2002). Consequently, increases in CK activity and myoglobin concentration within the blood are often used as indirect markers of muscle damage in exercise studies (e.g., Thompson et al., 2001a; Peake et al., 2005). Additionally, subjective ratings of muscle soreness have been extensively used to monitor the development and progression of delayed onset of muscle soreness that often follows exercise associated with muscle damage (e.g., Thompson et al., 2001a; Miller et al., 2004; Peake et al., 2005).

Employing a double-blind cross over study design, Fahey and Pearl (1998) reported that oral administration of S-PtdSer $\left(800 \mathrm{mg} \cdot \mathrm{d}^{-1}\right)$ during two weeks of intense weight training did not significantly attenuate serum $\mathrm{CK}$ activity when compared with the placebo; nevertheless, these authors did report that S-PtdSer reduced subjective ratings of muscle soreness during training. It has been suggested that mechanical damage leads to the removal of the most susceptible muscle fibres (Friden et al., 1983), through phagocytosis and repair. Therefore, it remains plausible, as suggested by these authors, that S-PtdSer supplementation might have influenced the structural integrity of the muscle cell in the vulnerable muscle fibres. 


\subsection{EFFECTS OF EXERCISE ON PHOSPHATIDYLSERINE CONTENT WITHIN CELL MEMBRANES}

Tibbits et al. (1981) reported that extended exercise training increased the levels of phospholipids, especially PtdSer content, in rat cardiac sarcolemma. This adaptation to training suggests that additional PtdSer within the heart muscle has functional benefits during exercise. An increase in membrane bound PtdSer might have the potential to enhance myocardial excitation-contraction coupling, possibly through the activation of different protein kinase $\mathrm{C}$ isoforms (Sugden and Bogoyevitch, 1995) and/or enhanced calcium uptake (Pepeu et al., 1996).

To the contrary, Liang et al. (1993) demonstrated that exercise training (10 weeks of intense aerobic training) led to significant reductions in the PtdSer content of predominantly oxidative skeletal muscles (soleus), while no changes were observed in predominantly anaerobic skeletal muscles (plantaris) in rats. Additionally, short term endurance exercise training has been reported to reduce the rate of PtdSer remodelling in rat skeletal muscle when compared with the control animals (Gorski et al., 1999). These findings suggest that additional PtdSer is required during exercise training to maintain the normal PtdSer content within the inner membrane of skeletal muscle, at least in rats.

Sumidikawa et al. (1993) showed that trained students (rowers that participated in regular training $3 \mathrm{~h}$ or more a day $\left(6 \mathrm{~d} \cdot \mathrm{week}^{-1}\right)$ for at least 2 years $)$ had significantly lower erythrocyte PtdSer when compared with control students of similar age, stature and body mass. Interestingly, these authors also found that a maximal exercise test led to selective reductions in the PtdSer content of erythrocyte 
membranes in the untrained group only. These data demonstrate that undertaking acute and chronic exercise lessens the PtdSer content of erythrocyte membranes. However, as blood samples were only taken before and after maximal exercise, the time course for the reduction and subsequent recovery of erythrocyte PtdSer membrane content following acute exercise was not available. Furthermore, as chronic endurance training was shown to reduce the PtdSer content in erythrocytes, it would appear that de novo synthesis was not able to restore 'normal' resting concentrations. Accordingly, it is plausible that exogenous S-PtdSer might allow the maintenance of PtdSer concentrations in cell membranes under the stress of acute and chronic exercise. 


\subsection{RESEARCH AIMS}

Phosphatidylserine is a ubiquitous component of the plasma membrane in eukaryotic cells, which has been demonstrated to influence cellular functions (as described earlier in this chapter), such as: cell excitability and communication; neuroendocrine responses; responses to inflammation; and antioxidant actions. The ability of PtdSer to moderate this array of cellular functions has been suggested to explain the findings that PtdSer supplementation enhances cognitive function in humans and other mammals. S-PtdSer supplements are considered a safe method of enhancing circulatory and tissue concentrations of PtdSer. Indeed, S-PtdSer is commercially available as an oral supplement that is intended to improve cognitive function.

Exercise poses wide-ranging challenges to cellular function; consequently, it is plausible that enhancing the availability of PtdSer might enhance cellular function during exercise. Few studies have investigated the effects of exogenous PtdSer on physiological function during exercise. Nevertheless, the available data suggest that supplementation with S-PtdSer modifies neuroendocrine function by attenuating the exercise induced activation of the HPA axis. In addition, oral supplementation with S-PtdSer has been reported to reduce perceived soreness and improve well-being after exercise (Fahey and Pearl, 1998); however, the mechanisms responsible for these results were unclear. Although not evaluated in this study, it is possible that antioxidant and/or anti-inflammatory actions of the supplement could explain these findings. Furthermore, at the commencement of this research, no studies had evaluated the in-vivo effects of S-PtdSer supplementation on oxidative stress or exercise performance/capacity during exercise. 
The series of experiments contained within this thesis were designed to investigate the following aims:

i In light of in-vitro research that suggests antioxidant functions of PtdSer, the purpose of the first experimental study was to investigate the effects of shortterm oral S-PtdSer on antioxidant status, tissue oxidation, muscle damage, and muscle soreness following exhaustive prolonged intermittent exercise in familiarised active participants. Hypotheses are located in section 3.1.

ii As supplementation tended to improve exercise performance in the first experimental study, it was difficult to evaluate the effects of supplementation on exercise induced oxidative stress. Consequently, the exercise protocol in the second experimental study was designed to cause repeatable amounts of muscle damage and oxidative stress. The purpose of this study was to investigate the proposed antioxidant and anti-inflammatory effects of shortterm S-PtdSer supplementation on markers of muscle damage, inflammation and lipid peroxidation following prolonged downhill running. Hypotheses are located in section 4.1 .

iii The third experimental chapter was designed to investigate the potential ergogenic effects of supplementation that were identified in experimental study one. Therefore, this study was designed to investigate the efficacy of short-term S-PtdSer supplementation on exercise capacity, neuroendocrine 
function, oxygen uptake kinetic response and perceived feeling states during and following intermittent cycling. Hypotheses are located in section 5.1. 
CHAPTER TWO

\section{GENERAL METHODS}




\section{GENERAL METHODS}

This chapter describes the general methods and procedures that were used throughout the experimental studies (Chapters 3 to 5 ) contained within this thesis. Specific methods and procedures that are not presented within this section are described in the methods sections within the relevant experimental chapters.

\subsection{SUBJECTS AND STUDY APPROVAL}

All of the experimental studies described within this thesis (Chapters 3 to 5) involved human volunteers. The subject groups that participated in this thesis were: university standard male soccer players (Chapter 3 ) and active young males (Chapter 4 and Chapter 5). The inclusion and exclusion criteria for each study are presented in the relevant experimental chapters.

Prior to undertaking each experimental study, approval was sought and gained from an ethical committee of the University of Wales Swansea (Appendix A). All participants received verbal and written information (Appendix B) that explained the purpose and requirements of the study that they volunteered to undertake. After completing health questionnaires (Appendix C), all subjects provided written informed consent (Appendix D) before taking part in any investigation. In addition, the subjects were made aware of their right to withdraw from the experiment at any stage without explanation. 


\subsection{EXPERIMENTAL DESIGN}

Experimental studies one and three followed a double-blind placebo controlled design. The subjects within each supplementation group were matched by measured (study three) or predicted (study one) maximum oxygen uptake $\left(\dot{\mathrm{VO}}_{2}\right.$ max $)$. The main limitation to this design is the use of subjects as their own control. However, the washout kinetics of PtdSer from human tissues are yet to be defined; consequently, it might be difficult to interpret the results from crossover studies, where some subjects will receive the placebo during the second trial and PtdSer concentrations within various tissues might remain elevated from the previous supplementation period.

A double-blind placebo controlled crossover design was used in study two. However, due to the aforementioned problem associated with this study design, control trials were included prior to both supplementation trials (PS and placebo). Consequently, a lack of difference between data from these pre-supplementation trials was interpreted to confirm that pre-supplementation data were similar; hence, the washout period provided was deemed to be sufficient for these data.

Familiarisation trials were included in all experiments in order to reduce the effect of trial order. The details of these trials are included in the methods section within each experimental chapter (Chapters 3 to 5). 


\subsection{SUPPLEMENTATION}

All of the experimental studies (Chapters 3 to 5) describe placebo controlled oral PtdSer supplementation studies. The composition of the PtdSer supplement and placebo were consistent throughout these chapters.

The method used to manufacture PtdSer (Lucas Meyer, Germany) makes use of the enzymatic transesterification of soybean lecithin. During this process, the headgroup of the starting phospholipids, obtained from the soybean lecithin (mainly phosphatidylcholine and phosphatidylethanolamine), are converted to PtdSer. Although the total amount of phospholipids remains unchanged, the concentration of PtdSer in this product is in the range of $45-54 \%$. The variation in concentration is mainly due to differences in the composition of the starting soybean lecithin. Subsequently, this product is blended with soybean lecithin to provide a final supplement, as used throughout the experimental sections of this thesis, which contains approximately $20 \%$ PtdSer. Table 2.1 shows the typical composition of the concentrated PtdSer product. The remaining content reflects the composition of the source soybean lecithin. Lecithin is usually used as a synonym for phosphatidylcholine; although, the composition of lecithin is variable depending upon the source soybean. Soybean lecithin also contains, to a lesser extent, a mixture of phospholipids, tocopherols, triglycerides, and free fatty acids. The approximate content of the final product is presented in Table 2.2.

Weight matched placebo capsules (containing a glucose polymer) were provided in generic packaging to ensure that the supplements were delivered to all participants 
Table 2.1: Typical composition of concentrated phosphatidylserine product.

\begin{tabular}{ll}
\hline Phospholipids & Composition \\
\hline Phosphatidylserine & $45-54 \%$ \\
Phosphatidylcholine & $5-7 \%$ \\
Phosphatidylethanolamine & $6-10 \%$ \\
Phosphatidylinositol & $1-3 \%$ \\
Phosphatidic acid & $4-7 \%$ \\
\hline
\end{tabular}


Table 2.2: Approximate composition of final phosphatidylserine product.

\begin{tabular}{ll}
\hline Phospholipids & Composition \\
\hline Phosphatidylserine & $20 \%$ \\
Phosphatidylcholine & $9 \%$ \\
Phosphatidylethanolamine & $2 \%$ \\
Phosphatidylinositol & $2 \%$ \\
Linoleic Acid & $23 \%$ \\
Linolenic Acid & $2 \%$ \\
Oleic Acid & $2 \%$ \\
Stearic Acid & $<1 \%$ \\
Palmitic Acid & $5 \%$ \\
Capric Acid & $10 \%$ \\
Caprylic Acid & $26 \%$ \\
Phosphorus & $2 \%$ \\
Potassium & $<1 \%$ \\
Others (including tocopherol) & Not listed \\
\hline
\end{tabular}


in a double blind manner. During the supplementation period the subjects were instructed to consume a total of 10 capsules per day with food ( 3 in morning, 3 at midday and 4 in evening). None of the subjects reported any side effects.

\subsection{DIETARY ANALYSIS}

All subjects were requested to weigh and record their dietary intake on three consecutive days during each main trial (beginning on the day prior to each exercise test). Subsequently, energy intake and dietary composition were determined for each subject using a commercially available computerised programme (CompEat version 5.8.0; Nutrition Systems, UK). These results were used to ensure that any changes in performance and/or antioxidant vitamins were not the result of changes in dietary intake.

\section{$2.5 \quad$ ANTHROPOMETRY}

Body mass and height were recorded in accordance with the International Standards for Anthropometric Assessment (ISAK, 2001) published by the International Society for the Advancement of Kinanthropometry. Body mass (BM) was determined using beam balance scales (model 710; Seca, UK). The subjects were dressed in minimal clothing and stood on the centre of the platform with their body weight evenly distributed on both feet (recorded to the nearest $0.05 \mathrm{~kg}$ ). Free standing height was determined using a stadiometer (Harpenden Portable Stadiometer; Holtain, UK) to 
the nearest $0.001 \mathrm{~m}$. The subjects stood with feet together and heels touching the scale as the head board of the stadiometer was lowered to the vertex of the head, which was placed in the Frankfort plane.

\subsection{EXPERIMENTAL CONTROLS}

The subjects were asked to refrain from undertaking strenuous activities before and after each exercise trial. The time period for this request during each experimental study are described within the specific methods section contained within each experimental chapter (Chapters 3 to 5). This experimental control was included in order to reduce the influence of additional activities on performance and markers of muscle damage, inflammation, oxidative stress and soreness. All of the subjects gave their verbal assurance that they had complied with these instructions.

\subsection{BLOOD ANALYSES}

Venous blood was obtained using venepuncture (Vacutainer system; BectonDickinson Ltd, UK) from an antecubital vein during all of the experimental studies described in this thesis (Chapters 3 to 5). The explicit procedures of blood preparation and blood analyses used in each experiment are described in the specific methods sections contained within Chapters 3 to 5 . This section provides a description of the principle and precision of each assay employed during the experimental chapters of this thesis. 
Small aliquots of whole blood were removed for the triplicate determination of blood lactate concentration and glucose concentration using an automated electrochemical analyser (YSI 2300, Yellow Springs Instruments, USA). Both assays are based on the principle that the substrate is rapidly oxidased to form hydrogen peroxide $\left(\mathrm{H}_{2} \mathrm{O}_{2}\right)$ when it comes in contact with the specific immobilised oxidase enzyme (Reaction 1). The $\mathrm{H}_{2} \mathrm{O}_{2}$ produced, is in turn, oxidised by the platinum anode which produces electrons (Reaction 2); consequently, the electron flow is linearly proportional to the substrate, as follows:

Reaction 1: $\quad \beta$-D-glucose $+\mathrm{O}_{2} \Rightarrow$ Glucoono- $\delta$-lactone $+\mathrm{H}_{2} \mathrm{O}_{2}$

(Glucose) Immobilised oxidase enzyme

Reaction 2: $\quad \mathrm{H}_{2} \mathrm{O}_{2} \Rightarrow 2 \mathrm{H}^{+}+\mathrm{O}_{2}+2 \mathrm{e}^{-}$

Platinum electrode

The precision of measurement across the measurement ranges for blood lactate and glucose concentrations had coefficients of variation (CV) of 1.4 and $1.0 \%$, respectively. 
Estimated changes in plasma volume (\%) were calculated from measured haemoglobin and haematocrit values, using the method described by Dill and Costill (1974).

(a) Haemoglobin

Haemoglobin concentrations were determined (in triplicate) from whole blood using an automated 2-wavelength $(570$ and $880 \mathrm{~nm})$ spectrophotomer (B-Hemoglobin Analyser; Hemocue Ltd, UK) as described by the manufacturers instructions. The precision of measurement across the range of values had a $\mathrm{CV}$ of $1.0 \%$.

\section{(b) Haematocrit}

Haematocrit was determined in triplicate using standard microhaematocrit tubes. Each tube was filled with blood and centrifuged for $5 \mathrm{~min}$ at $12000 \mathrm{x} \mathrm{g}$ (Micro Haematocrit MK IV, Hawksley, UK). A Hawksley reader was used to quantify the packed cell volume (haematocrit \%). The precision of measurement across the range of values had a CV of $0.9 \%$. 
Creatine kinase (CK) activities were determined using an automated spectrophotometer (MIRA S; ABX Diagnostics, UK) with commercially available kits (kit A11A00008; ABX Diagnostics, UK). The $\mathrm{CK}$ is activated by $\mathrm{N}$ acetylcysteine and determined by the following reaction sequence:

Reaction 1: Creatine phosphate $+\mathrm{ADP} \Rightarrow$ creatine + ATP

\section{CK}

Reaction 2: $\quad$ ATP + D-glucose $\Rightarrow$ ADP + D-glucose-6-P

$H K$

Reaction 3: $\quad$ D-glucose-6-P $+\mathrm{NADP}^{+} \Rightarrow$ D-gluconate-6-P $+\mathrm{NADPH}+\mathrm{H}^{+}$ G6P-dehydrogenase

Where the rate of NADPH was measured photometrically at $340 \mathrm{~nm}$, and is directly related to the $\mathrm{CK}$ activity. The intra and inter assay CVs, across the measurement range, were 0.7 and $0.9 \%$, respectively.

Determination of Myoglobin Concentrations

Myoglobin concentrations were determined using an automated spectrophotometer (MIRA S; ABX Diagnostics, UK) with commercially available kits (kit A11A00165; ABX Diagnostics, UK). The assay is based on the principle that a clear agglutination is formed when myoglobin contained within the sample is mixed with the Myoglobin Lated reagent. This product was measured by turbidimetric 
immunoassay. The intra and inter assay $\mathrm{CVs}$, across the measurement range, were 3.8 and $4.8 \%$, respectively.

Determination of Cortisol Concentrations

Experimental studies 1 and 3 (Chapters 3 and 5) used an automated time-resolved flouroimmunoassay (AutoDELFIA ${ }^{\mathrm{TM}}$ Cortisol kit, Perkin Elmer, Life Sciences, UK) to determine serum total cortisol concentrations. Plasma total cortisol concentrations were determined using enzyme-linked immunosorbent assays (ELISA) during experiment 2 (Chapter 4).

(a) Flouroimmunoassay

The assay is a solid phase time resolved flouroimmunoassay and is based on the principle that europium chelate $(\mathrm{Eu})$ labelled cortisol competes for a fixed time with sample cortisol for a limited number of cortisol specific, biotinylated monoclonal antibodies. Washing off the supernatant terminates the competition and leaves the antibody-bound cortisol fraction immobilised to the wall of the polypropylene tube. After an enhancement solution has been added a new flourescent chelate is formed and measured on the automated flourometer. Using a calibration curve, the radioactivity in the tube can be converted to quantify the cortisol present within the sample. The intra and inter assay precisions had CVs of $<10 \%$ across the range of values undertaken. 
(b) ELISA

This solid-phase ELISA was performed in duplicate using commercially available kits (kit EIA-1887; DRG Instruments, Germany) on a spectrophotometric microplate reader (BP800; Biohit, UK). The assay is based on the principle that horseradish peroxidase-labelled cortisol (HRP-cortisol) competes with cortisol present in the sample for a fixed and limited number of antibody sites that are immobilised on the wells of the pre-prepared microstrips. Once the competitive immunoreaction had occurred, the wells were rinsed using an automated plate washer (BW50; Biohit, UK), and the HRP-cortisol fraction bound to the antibody in the solid phase was measured by adding a chromogen/substrate solution that produced a blue coloured compound. After 15 min of incubation, the enzymatic reaction was stopped with hydrochloride acid, which changed the solution to a yellow colour.

The absorbance of each solution was photometrically measured at $450 \mathrm{~nm}$. Total cortisol concentrations were calculated with reference to calibration curves that were developed for each 96 well microplate. The intra assay precision across the measurement range had a CV of $4.5 \%$. 
Plasma adrenocorticotropic hormone (ACTH) concentrations were determined in duplicate using solid-phase enzyme-linked immunosorbent assays (ELISA) with commercially available kits (kit 7023; Biomerica, US). This two-site ELISA uses a goat polyclonal antibody to human $\mathrm{ACTH}$ and a mouse monoclonal antibody to human ACTH, which are specific for well defined regions on the ACTH molecule. One antibody is prepared to bind only the C-terminal ACTH 34-39 and this antibody is biotinylated. The other antibody is prepared to bind only to the mid-region and $\mathrm{N}$ terminal ACTH 1-24 and this antibody is labelled with horseradish peroxidase for detection. The samples were simultaneously incubated with the enzyme labelled antibody and a biotin coupled antibody in the pre-prepared streptavidin-coated microplate wells. At the end of the assay incubation, the microwells were washed (BW50; Biohit, UK) to remove unbound components and the enzyme bound to the solid phase were incubated with the substrate, tetramethylbenzidine. Sulphuric acid was then added to stop the reaction and convert the colour to yellow.

The intensity of the yellow colour in each microwell was read at $450 \mathrm{~nm}$ (BP800; Biohit, $\mathrm{UK}$ ) and $\mathrm{ACTH}$ concentrations were determined using calibraton curves that were developed for each 96 well plate. The intra assay precision across the measurement range had a $\mathrm{CV}$ of $8.1 \%$. 
Interleukin-6 (IL-6) concentrations in serum were assayed in duplicate using commercially available high sensitivity ELISA kits (Diaclone Research, France). The samples were added to wells within each microplate that were pre-coated with a monoclonal antibody specific for IL-6. The IL-6 antigen and a biotinylated monoclonal antibody specific for IL-6 were incubated for $3 \mathrm{~h}$. After washing (BW50; Biohit, UK), the enzyme (streptavidin-peroxydase) was added. After a second incubation (30 min) and washing (BW50; Biohit, UK), a substrate solution (tetramethylbenzidine), which acts on the bound enzyme, was added to induce a coloured reaction product. Sulphuric acid was then added to stop the reaction and convert the colour to yellow.

The intensity of this yellow coloured product was read at $450 \mathrm{~nm}$ (BP800; Biohit, UK) and IL-6 concentrations were determined using calibraton curves that were developed for each 96 well plate. The intra assay precision across the measurement range had a CV of $12.1 \%$. 
Serum hydroperoxide concentrations were measured using the method of Wolff (1992) as described in McEneny et al. (1998). Serum (90 $\mu \mathrm{L})$ was incubated with 10 $\mu \mathrm{L}$ of triphenylphosphine $\left(10 \mathrm{mmol} \cdot \mathrm{L}^{-1}\right)$ for $30 \mathrm{~min}$. Subsequently, $900 \mu \mathrm{L}$ of FOXII reagent $(250 \mu \mathrm{M}$ ammonium ferrous sulphate, $100 \mu \mathrm{M}$ xylenol orange, $25 \mathrm{mM}$ $\mathrm{H}_{2} \mathrm{SO}_{4}$ and $4 \mathrm{mM}$ butylated hydroxytoluene in $90 \%$ methanol) was added to the test sample. Samples and standards were incubated for $30 \mathrm{~min}$ at room temperature and centrifugation in a Beckman microfuge for $5 \mathrm{~min}$. The absorbance of the supernatant was read at $560 \mathrm{~nm}$.

Hydroperoxides oxidise ferrous ions to ferric ions in dilute acids and the resultant ferric ions can be determined using ferric-sensitive dyes as an indirect measure of hydroperoxide concentration, using the following principle:

$$
\begin{array}{ll}
\text { Reaction 1: } & \begin{array}{c}
\mathrm{Fe}^{2+}+\mathrm{ROOH} \\
\text { (peroxides) }
\end{array} \\
\text { Reaction 2: } & \mathrm{Fe}^{3+}+\mathrm{OH}^{-}+\text {Sorbitol } \Rightarrow \text { (oxyl radicals) } \\
\text { Reaction 3: } & \mathrm{HO}_{2}{ }^{-}+\mathrm{Fe}^{2+} \Rightarrow \mathrm{Fe}^{3+}+\mathrm{H}_{2} \mathrm{O}_{2}
\end{array}
$$

The inter and intra assay precision, across the measurement range, had CVs less than $5 \%$. 
Low density lipoprotein was isolated from plasma and oxidised according to the method of McDowell et al. (1995). Low density lipoprotein (LDL) was isolated using a density gradient method with a Beckman TL100 ultracentrifuge (Beckman Instruments, UK). Plasma $(0.9 \mathrm{~mL})$ was added to a centrifuge tube containing potassium bromide ( $0.4451 \mathrm{~g}$; Sigma, UK) and gently mixed until the salt dissolved. Subsequently, $2.1 \mathrm{~mL}$ of $0.15 \mathrm{~mol} \cdot \mathrm{L}^{-1} \mathrm{NaCl}$ was added and centrifuged at 100000 rpm for $60 \mathrm{~min}$ at $4^{\circ} \mathrm{C}$. The density gradient forms a layer of LDL that was carefully removed with a 21 gauge hypodermic needle. The preparation containing LDL was then desalted using gel filtration at $4^{\circ} \mathrm{C}$ using columns containing Sephadex G25 (PD-10; Pharmacia Ltd, UK). Additionally, albumin and salt were removed by gel filtration at $4^{\circ} \mathrm{C}$ using column packed with Sephacryl $300 \mathrm{HR}$ (C10-20; Pharmacia Ltd, UK). Fractions were collected at 1 min intervals.

The production of conjugated dienes was followed in duplicate at $234 \mathrm{~nm}$ (SpectraMax 190; Molecular Devices Corp, US) using the computer software SoftMax Pro Version 3.0 (Molecular Devices Corp, US); the change in absorbance (from baseline to the end of reaction) was used to quantify the change in conjugated diene concentration.

The lag times were used as a measure of the resistance of the particle to oxidation in experimental study 1 . Lag times are taken as the intercept between absorbance at time zero and the point on the curve when maximum LDL oxidation occurred (Figure 2.1). 
The time taken to reach half of the maximum oxidation ( $t_{1 / 2 \max }$ LDL oxidation) was taken as a measure of resistance of the particle to oxidation in experimental study 2. The $t_{1 / 2 \max }$ is the time taken to reach half of the maximum change in conjugated diene production (Figure 2.1). 


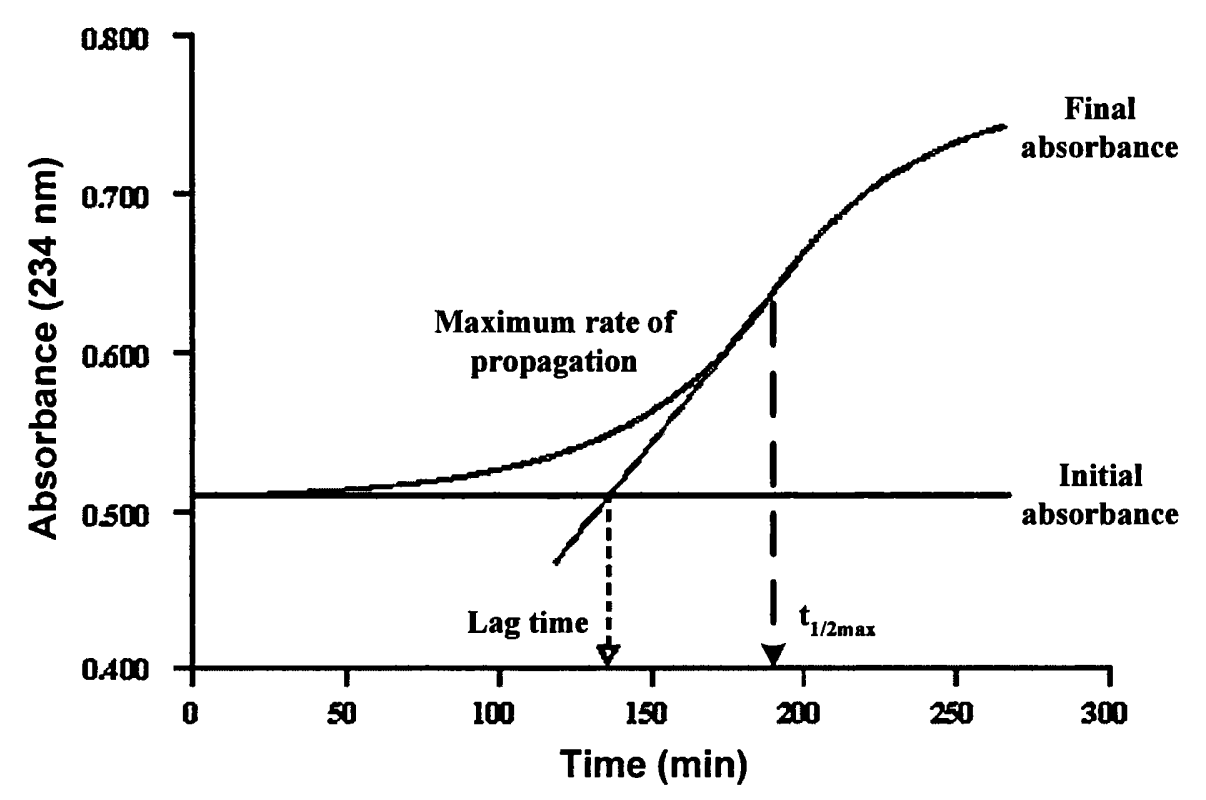

Figure 2.1 Low density lipoprotein (LDL) oxidation, displaying the identification of conjugated diene lag time and time to half maximum LDL oxidation $\left(t_{1 / 2 \max }\right)$. 
Fresh aliquots of plasma $(100 \mu \mathrm{L})$ were added to $900 \mu \mathrm{L}$ of freshly prepared $10 \%$ metaphosphoric acid, mixed and frozen at $-70^{\circ} \mathrm{C}$ for subsequent vitamin $\mathrm{C}$ analysis. Vitamin $\mathrm{C}$ concentrations were determined by means of a fluorimetric assay using a centrifugal analyser (Cobas Fara) with fluorescence attachment according to the method of Vuilleumier (1993).

This procedure is based on the enzymatic oxidation of ascorbic acid and subsequent quinoxaline formation to generate a fluorescent derivative measured on the centrifugal analyser. Aliquots of standards and samples $(200 \mu \mathrm{L})$ were placed in Cobas cups. Oxidising reagent $\left(9.9 \mathrm{ml}\right.$ of $2 \mathrm{~mol} \cdot \mathrm{L}^{-1}$ acetate buffer and stock ascorbate oxidase) and coupling reagent (20 $\mathrm{mg}$ of 1,2-phenylenediamine dissolved in $20 \mathrm{ml}$ deionised water) were added to appropriate reagent wells. The automated assays were then started. The intra assay CVs at mean vitamin $\mathrm{C}$ concentrations of $4.2,50.6$ and $151.0 \mu \mathrm{mol} \cdot \mathrm{L}^{-1}$ were $6.8,0.72 \%$ and $1.50 \%$, respectively.

Determination of Plasma Vitamin A and D concentrations

Plasma concentrations of $\alpha$-tocopherol, $\gamma$-tocopherol, retinol, $\alpha$-carotene, $\beta$-carotene and lycopene were measured by high-performance lipid chromatography (HPLC) with electrochemical detection according to the methods of Catignani and Bieri (1983) and Thurnham et al. (1988). 
Plasma samples and standards $(300 \mu \mathrm{L})$ were pipetted into glass tubes $(4 \mathrm{~mL})$. Ethanol $(250 \mu \mathrm{L})$ containing the internal standards (e.g., $\alpha$-tocopherol acetate) and $0.25 \mathrm{~g} \cdot \mathrm{L}^{-1}$ butylated hydroxy toluene was added. Subsequently, $500 \mu 1$ heptane was added and the tubes were vortexed vigorously for $1 \mathrm{~min}$ to extract the lipid soluble vitamins into the heptane. The sample tubes were then centrifuged for $5 \mathrm{~min}$ at 1200 $\mathrm{x} \mathrm{g}$ and $350 \mu \mathrm{L}$ of the supernatant were transferred into clean glass tubes. The original tube was re-extracted and a further $350 \mu \mathrm{L}$ of supernatant was removed and added to the first extraction giving a final volume of $700 \mu \mathrm{L}$. Theses tubes were evaporated to dryness in a rotary evaporator under vacuum for $1.2-2 \mathrm{~h}$. These samples were frozen at $-70^{\circ} \mathrm{C}$ until they were analysed by HPLC. Samples and standards were reconstituted in $150 \mu \mathrm{L}$ methanol, vortexed and assayed by HPLC using a diode array detector (Thermo Separation Products). Samples and standards were injected onto a Hypersil ODS $5 \mu \mathrm{m}$ column. The mobile phase contained $97 \%$ methanol: $3 \%$ tetrahydrofurin. The intra assay CVs for these assays ranged from $1.4 \%$ for $\alpha$-tocopherol to $12 \%$ for lycopene. 


\section{CHAPTER THREE}

\section{EXPERIMENTAL STUDY ONE}

Effects of phosphatidylserine supplementation on performance and oxidative stress following intermittent running 


\subsection{INTRODUCTION}

Soccer is a multiple-sprint sport that requires high-intensity, intermittent activity to be undertaken over an extended period of time (approximately $90 \mathrm{~min}$ ). The rapid decelerations and changes in direction undertaken during soccer match play generate large eccentric forces that have frequently been associated with muscle damage (Friden et al., 1983), leakage of unbound iron from the cytosolic region (Jenkins $e t$ al., 1993) and delayed onset of muscle soreness (DOMS) (Clarkson et al., 1992). Free radical production has been associated with muscle damaging exercise (Aoi $e t$ al., 2004) and the ensuing inflammatory response (Macintyre et al., 1995). In addition, the elevation in oxygen consumption and the rapid changes in mitochondrial oxygen flux that are associated with prolonged intermittent exercise have the potential to greatly increase the production of free radicals (Dekkers et al., 1996). These radicals have the potential to cause oxidative damage to a wide range of molecular structures including lipids, proteins and DNA, where the antioxidant defences are overwhelmed (Kanter et al., 1988).

Consequently, the mechanisms responsible for muscle damage include primary damage caused by direct mechanical stress and/or elevated oxygen uptake and secondary damage caused by autogenic processes that lead to inflammation and phagocytosis. Although it is evident that activities involving substantial amounts of eccentric muscular activity accompanied with elevated oxygen uptake have potential to cause muscle damage, it is not possible to separate the potential mechanisms that lead to this phenomenon. 
The symptoms of exercise-induced muscle damage often include: soreness, decrease in the range of motion of joints in affected limbs, decreased muscular strength, decreased power output, leakage of myofibre proteins, inflammation and oedema (Kendall and Eston, 2002). Furthermore, prolonged intermittent shuttle-running designed to simulate multiple-sprint sports has been previously demonstrated to cause muscle soreness, and elevate blood markers of muscle damage and lipid peroxidation in accustomed individuals (Thompson et al., 2001a).

Phosphatidylserine (PtdSer) is an essential phospholipid, mainly located within the cytosolic monolayer (Devaux, 1991), that has been shown to have important regulatory functions within mammalian cells (Blokland et al., 1999). Exogenous PtdSer supplementation has shown benefits in diverse measures of cognitive functions. Furthermore, intravenously and orally administered bovine cortex-derived PtdSer have been shown to blunt the cortisol response to acute exercise stress (Monteleone et al., 1990; Monteleone et al., 1992) and, therefore, might attenuate the stress-induced activation of the hypothalamo-pituitary-adrenal axis in man. Moreover, Fahey and Pearl (1998) reported that short-term oral supplementation with $800 \mathrm{mg} \cdot \mathrm{d}^{-1}$ soybean derived PtdSer (S-PtdSer) was effective in reducing serum cortisol concentrations and muscle soreness following intensive resistance training. In addition to a myriad of membrane related functions, in-vitro studies have demonstrated that PtsSer has the potential to act as an antioxidant (Latorraca et al., 1993; Dacaranhe and Terao, 2001). Therefore, it is plausible that exogenous supplementation with PtdSer might provide additional defence against the oxidative stress caused by soccer match play. In light of the above, the aim of the present study was to determine whether antioxidant status, tissue oxidation, muscle damage, 
and muscle soreness following exhaustive prolonged intermittent exercise would be affected by short-term oral S-PtdSer supplementation in accustomed active university soccer players.

\section{Null Hypotheses}

$\mathrm{HO}_{1}$ : Exhaustive intermittent running will not influence markers of antioxidant status, tissue oxidation, muscle damage, and muscle soreness.

$\mathrm{HO}_{2}$ : Supplementation with S-PtdSer will not influence markers of antioxidant status, tissue oxidation, muscle damage, and muscle soreness after exhaustive intermittent running.

$\mathrm{HO}_{3}$ : Supplementation with S-PtdSer will not influence neuroendocrine responses to exhaustive intermittent running.

$\mathrm{HO}_{4}$ : Supplementation with S-PtdSer will not influence exercise performance during exhaustive intermittent running. 


\subsection{METHODS}

\section{Subjects}

Sixteen young males who participated regularly in soccer match play and practice volunteered to participate in this study, which was approved by a university ethics committee. All subjects were informed in writing about the potential risks of the study and gave written informed consent for their participation in the study. No subject had prior history of cardiovascular or respiratory disease and all subjects were non-smokers. Potential subjects attended an interview prior to undertaking the study and were subsequently excluded if they were smokers or had taken nutritional supplements in the last 8 weeks. The subjects' physical characteristics are presented in Table 3.1.

\section{Experimental Design}

All subjects completed two preliminary visits to the laboratory prior to undertaking the main exercise trials. Maximum oxygen uptake ( $\left.\dot{\mathrm{V}}_{2} \max \right)$ was estimated using a multistage fitness test (MSFT) (Ramsbottom et al., 1998) during the initial visit. These $\dot{\mathrm{VO}}_{2} \max$ values were used in conjunction with the linear regression developed by Ramsbottom et al. (1988), to calculate appropriate running speeds for the exercise protocol as described previously by Nicholas et al. (2000). 
Table 3.1 Subject characteristics for phosphatidylserine supplementation group (PS) and placebo group $(\mathrm{P})$.

\begin{tabular}{lccc}
\hline Characteristic & PS (N=8) & P (N=8) & P-value \\
\hline Age (dec. years) & $21.7 \pm 0.3$ & $22.6 \pm 0.7$ & 0.320 \\
Mass (kg) & $81.7 \pm 2.4$ & $74.0 \pm 2.4$ & 0.042 \\
Height (m) & $1.82 \pm 0.03$ & $1.80 \pm 0.04$ & 0.714 \\
Estimated maximal oxygen uptake $\left(\mathrm{ml}^{-\mathrm{kg}^{-1}} \mathrm{~min}^{-1}\right)$ & $52.8 \pm 2.1$ & $54.7 \pm 1.7$ & 0.493 \\
\hline
\end{tabular}

Values are mean \pm SEM. $P$-value calculated using independent sample $t$-test. 
During their second preliminary visit the subjects underwent a familiarisation exercise trial in order to minimise anticipated trial order effects. Subjects then performed two main exercise trials (T1 and T2), separated by $14 \pm 1 \mathrm{~d}$. The subjects were assigned, in a randomised double-blind fashion, to receive either $750 \mathrm{mg} \cdot \mathrm{d}^{-1} \mathrm{~S}$ PtdSer (PS) or a weight matched glucose polymer (P) for exactly 10 days prior to the T2. The S-PtdSer supplements were manufactured using the method of specific transesterification of soybean lecithin and then blended with additional soybean lecithin to provide a concentration of $20 \%$ PtdSer (Lucas Meyer; Hamburg, Germany). Both supplements were administered in capsules and placed in generic packaging. Subjects were instructed to maintain their normal diet and activity patterns throughout the study. Food was weighed and recorded by the subjects for the two days before and after each main exercise trial. These food records were subsequently analysed using commercial software (CompEat version 5.8.0; Nutrition Systems, UK). In addition, the subjects were instructed to abstain from strenuous exercise for three days prior to and two days following each trial. At the completion of the study, all subjects gave their verbal assurance that they had complied with all instructions.

\section{Main Trial Procedures}

On the day of both main exercise trials the subjects reported to the laboratory in pairs (matched by estimated $\dot{\mathrm{VO}}_{2}$ max and paired in different supplementation groups) at approximately the same time of day $( \pm 1 \mathrm{~h})$ and following an overnight fast. Subjects were asked to void, then mass (model 712; Seca, Germany) and height 
(Portable Stadiometer; Holtain, UK) were recorded. Subsequently, the intensity of muscle soreness for the whole body general soreness (GS), hamstrings (HS) and quadriceps (QS) were rated on an eleven-point scale ranging from 0 (not sore) to 10 (very, very sore) (Appendix E).

Following a Pre-exercise venepuncture (Vacutainer system; Becton-Dickinson Ltd, $\mathrm{UK}$ ), taken from an antecubital vein in the right arm (after maintaining a seated position for approximately $10 \mathrm{~min}$ ), the subjects completed an exercise protocol that consisted of two sections (Part A and Part B) in an indoor wooden floored gymnasium. Part A was based on the Loughborough Intermittent Shuttle Test (Nicholas et al., 2000), but included additional components designed to further replicate the physiological demands of soccer match play. Briefly, the protocol consisted of variable intensity shuttle running over a $20-\mathrm{m}$ distance that included walking, jogging $\left(55 \% \dot{\mathrm{VO}}_{2} \max \right)$, cruising $\left(85 \% \dot{\mathrm{VO}}_{2 \max }\right)$, backward cruising $\left(85 \% \dot{\mathrm{VO}}_{2} \max \right)$, zigzag sprinting, rest periods and timed sprinting (Brower; Utah, USA) undertaken in a repeated sequence dictated by pre-recorded audio compact discs. This sequence was undertaken for $45 \mathrm{~min}$, followed by a 10-min recovery period (half-time), and then continued for a subsequent 30-min exercise period. Immediately after the intermittent exercise, the MSFT was completed until volitional fatigue (Part B), thus the total exercise time was approximately $90 \mathrm{~min}$ (Figure 3.1).

Heart rates were continuously recorded each 5-s interval using short-range telemetry (Polar S610; Polar Electro, Finland). Subjective ratings of perceived exertion (RPE) and capillary blood samples were obtained at the beginning of the half-time recovery 

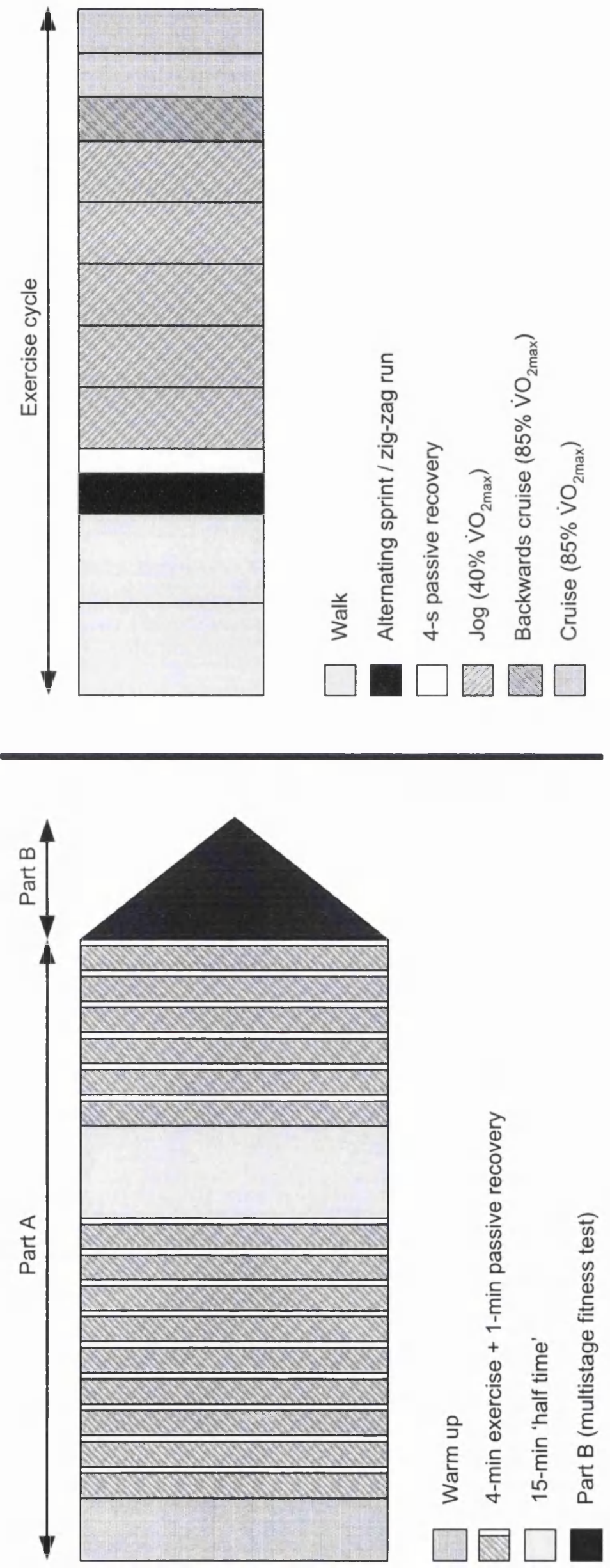

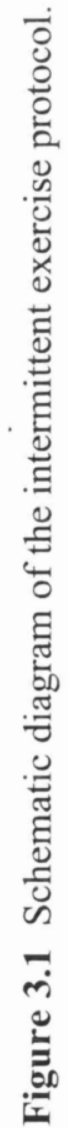


period (Half-time) and immediately following volitional fatigue (Post-MSFT). Ambient temperature and humidity were recorded at the beginning and end of exercise (ETHG-912; Oregan Scientific, USA). Ad Libitum water consumption was encouraged throughout $\mathrm{T} 1$; this volume was measured and each individual was required to match his fluid intake during T2. Fifteen min following the termination of exercise venous blood samples were taken, in a seated position, by venepuncture from an antecubital vein in the left arm as previously described.

On the day following (Post-24 h) and the second day following (Post-48 h) each main exercise trial the subjects returned to the laboratory at approximately the same time $( \pm 1 \mathrm{~h})$ after overnight fasts. Blood samples were drawn by venepuncture and the subjects rated the intensity of soreness as previously described.

\section{Blood Sampling and Analysis}

Venous blood was collected in a 5-mL container (Becton-Dickinson Ltd, UK) containing the anticoagulant ethylenediamine tetra-acetic acid (EDTA). Several small aliquots were removed for the triplicate determination of blood lactate and glucose concentrations (YSI 2300 Stat, Yellow Springs Instruments, USA), blood haemoglobin concentration (Hemocue limited, UK), haematocrit (Micro Haematocrit MK IV, Hawsley, England) and changes in plasma volume as previously described (Dill and Costill, 1974). The remaining blood was centrifuged at $3000 \mathrm{x}$ g for $15 \mathrm{~min}$ to obtain plasma. An aliquot of plasma $(100 \mu \mathrm{L})$ was added to $900 \mu \mathrm{L}$ of freshly prepared $10 \%$ metaphosphoric acid, mixed and frozen at $-70^{\circ} \mathrm{C}$ for 
subsequent vitamin $\mathrm{C}$ analysis. The remaining plasma was frozen and stored at $-70^{\circ} \mathrm{C}$ prior to subsequent analysis. Two additional $7-\mathrm{mL}$ blood samples were collected in serum separation tubes (Becton-Dickinson Ltd, UK), left to stand for 15 min and then centrifuged at $3000 \mathrm{x}$ g for $15 \mathrm{~min}$ to obtain serum. The serum was transferred to appropriate containers and subsequently stored at $-70^{\circ} \mathrm{C}$ before subsequent analysis.

Serum myoglobin $(\mathrm{Mb})$ concentrations and creatine kinase $(\mathrm{CK})$ activities were measured with commercial available kits (A11A00165 and A11A00008, respectively; ABX Diagnostics, UK) using an automated spectrometer (MiraS; ABX Diagnostics, UK). Serum cortisol concentrations were determined using an automated time-resolved fluoroimmunoassay (AutoDELFIA ${ }^{\mathrm{TM}}$ Cortisol kit, Perkin Elmer, Life Sciences, UK). Serum hydroperoxide (HPO) concentrations were measured using the method of Wolff (1992) as described in McEneny et al. (1998). Low density lipoprotein was isolated from plasma and oxidised according to the method of McDowell et al. (1995). Subsequently, the production of conjugated dienes was followed at $234 \mathrm{~nm}$ and the lag time taken as a measure of the resistance of the particle to oxidation. Vitamin $\mathrm{C}$ concentrations were determined using a fluorimetric assay using a centrifugal analyser with fluorescence attachment according to the method of Vuilleumier (1993). Plasma concentrations of $\alpha$-tocopherol, $\gamma$-tocopherol, retinol and $\beta$-carotene were measured by highperformance lipid chromatography with electrochemical detection according to the methods of Thurnham et al. (1988). 
Statistical analysis was carried out using SPSS software (version 12.0; SPSS Inc., IL). Group data were expressed as mean \pm SEM and statistical significance was set at the $\mathrm{P}<0.05$ level. Subject characteristics were compared under supplementation groups using independent samples $t$-tests (Table 3.1). Environmental conditions were compared using paired samples $t$-tests. The percentage change in exercise times to exhaustion during part B (individual delta values) was compared under PS and $\mathrm{P}$ conditions using an independent samples $t$-test. The remaining data, which contained multiple time points during each trial, were analysed using mixed-model repeated measures ANOVA (within subject factors: trial $\mathrm{x}$ time of sample; between subject factor: supplementation groups). Mauchly's test was consulted and Greenhouse-Geisser correction was applied if the assumption of sphericity was violated. If a significant $P$ value was identified for the interaction effect (supplementation group $\mathrm{x}$ trial $\mathrm{x}$ time of sample), supplementation was deemed to have a significant effect. If a significant $P$ value was identified for the main effect of time (time of sample), multiple pairwise comparisons were made using Bonferonni confidence interval adjustment, with statistical significance set at $\mathrm{P}<0.01$. 


\subsection{RESULTS}

The environmental conditions were not significantly different during all trials, ambient temperature being $19.6 \pm 0.7$ and $21.3 \pm 0.5^{\circ} \mathrm{C}$, and humidity $51.5 \pm 2.6$ and $54.0 \pm 2.8 \%$, respectively for $\mathrm{T} 1$ and $\mathrm{T} 2$. Mean and peak exercising heart rates were not significantly different for PS and P during T1 (PS, P: $151 \pm 6,153 \pm 6 ; 196 \pm 4$, $196 \pm 4$ beats $\mathrm{min}^{-1}$, respectively) and during T2 (PS, P: $149 \pm 5,149 \pm 7 ; 196 \pm 4$, $195 \pm 3$ beats $\min ^{-1}$, respectively). Blood lactate concentrations were similarly elevated from Pre-exercise $\left(1.3 \pm 0.1 \mathrm{mmol} \cdot \mathrm{L}^{-1}\right)$ at Half-time $\left(2.4 \pm 0.2 \mathrm{mmol} \cdot \mathrm{L}^{-1}\right)$, Post-MSFT $\left(6.1 \pm 0.2 \mathrm{mmol} \cdot \mathrm{L}^{-1}\right)$ during all trials $(P<0.01)$. Blood glucose concentrations were not significantly different for Pre-exercise, Half-time, PostMSFT and Post-exercise, being $4.2 \pm 0.1,4.5 \pm 0.1,5.3 \pm 0.1,4.0 \pm 0.1 \mathrm{mmol} \cdot \mathrm{L}^{-1}$, respectively for all trials. Ratings of perceived exertion were not different between trials at Half-time or at exhaustion, being $14 \pm 1$ and $18 \pm 1$ units, respectively for all trials. Estimated changes in plasma volume were not different between trials or at any point during the course of the data collection (Table 3.2). No differences were observed in the calculated amount or composition of the food consumed during any trial (Table 3.3). 
Table 3.2 Estimated percentage changes in plasma volume throughout both trials for phosphatidylserine supplementation group and placebo group.

\begin{tabular}{ccccccccc}
\hline & & & & & & & & \\
& T1 & T1 & T1 & T1 & T2 & T2 & T2 & T2 \\
& Pre-ex & Post-ex & Post-24 h & Post-48 h & Pre-ex & Post-ex & Post-24 h & Post-48 h \\
\hline PS & - & $2.0 \pm 2.5$ & $6.3 \pm 3.6$ & $4.0 \pm 2.1$ & $-3.6 \pm 2.1$ & $-2.8 \pm 1.5$ & $0.5 \pm 1.7$ & $4.1 \pm 3.1$ \\
P & - & $3.6 \pm 1.5$ & $6.3 \pm 1.9$ & $5.3 \pm 1.5$ & $-1.4 \pm 1.7$ & $0.5 \pm 1.4$ & $1.9 \pm 1.4$ & $5.1 \pm 1.6$ \\
\hline
\end{tabular}

Values are mean \pm SEM $(N=8)$. PS: phosphatidylserine group; $P$ : placebo group. T1: trial 1; T2: trial 2; Pre-ex: pre-exercise; Post-ex: post-exercise; Post- 24 h: 24 hours post-exercise; Post- 48 h: 48 hours post-exercise. 
Table 3.3 Energy intake and dietary composition during both exercise trials for phosphatidylserine supplementation group and placebo group.

\begin{tabular}{lcccccc}
\hline & $\begin{array}{c}\text { Energy } \\
\left(\mathbf{M J} \cdot \mathbf{d}^{-1}\right)\end{array}$ & $\begin{array}{c}\text { CHO } \\
(\% \text { energy) }\end{array}$ & $\begin{array}{c}\text { Fat } \\
(\% \text { energy })\end{array}$ & $\begin{array}{c}\text { Protein } \\
(\% \text { energy) }\end{array}$ & $\begin{array}{c}\text { Vit C } \\
\left(\mathbf{m g} \cdot \mathbf{d}^{-1}\right)\end{array}$ & $\begin{array}{c}\text { Vit E } \\
\left(\mathbf{m g} \cdot \mathbf{d}^{-1}\right)\end{array}$ \\
\hline PS Trial 1 & $11.4 \pm 0.9$ & $44 \pm 3$ & $30 \pm 3$ & $24 \pm 2$ & $57 \pm 13$ & $8 \pm 2$ \\
PS Trial 2 & $10.8 \pm 0.8$ & $42 \pm 2$ & $30 \pm 2$ & $23 \pm 1$ & $65 \pm 14$ & $8 \pm 1$ \\
P Trial 1 & $9.5 \pm 0.9$ & $43 \pm 1$ & $30 \pm 2$ & $25 \pm 2$ & $68 \pm 17$ & $8 \pm 2$ \\
P Trial 2 & $9.7 \pm 0.6$ & $45 \pm 1$ & $30 \pm 2$ & $24 \pm 1$ & $55 \pm 15$ & $8 \pm 2$ \\
\hline
\end{tabular}

Values are mean \pm SEM (N=8). PS: phosphatidylserine group; P: placebo group. Analysis does not include S-PtdSer supplementation. 
Exercise times to exhaustion during Part B of the exercise protocol were similar between the groups prior to supplementation (P: $11.3 \pm 0.6$; PS: $11.2 \pm 0.7 \mathrm{~min}$; $P=0.894$ ). There was a non-statistical tendency for the change (i.e, T2 - T1, expressed as a percentage of $\mathrm{T} 2$ ) in the exercise times to exhaustion to be longer in PS when compared with $\mathrm{P}$, being $4.2 \pm 0.7$ and $-3.7 \pm 4.2 \%$, respectively $(t=1.86$; $P=0.084$; Cohen's $d=0.92$ ) (Figure 3.2). Supplementation had no significant effect on sprint speeds during Part $\mathrm{A}$ of the exercise protocol (supplementation group $\mathrm{x}$ trial $\mathrm{x}$ time of sample, $P=0.143$ ). The changes (i.e. $\mathrm{T} 2$ - T1, expressed as a percentage of T2) in sprint speeds during sprints $1-6,7-11$ and $12-17$ were $3.7 \pm 1.3$, $4.1 \pm 1.4,7.3 \pm 2.6 \%$ and $3.7 \pm 2.8,4.4 \pm 2.8,0.4 \pm 2.8 \%$ for PS and P, respectively (Figure 3.3).

\section{Serum Cortisol Concentrations}

Pre-exercise, Post-exercise, Post-24 h and Post-48 h serum cortisol concentrations for all trials were $392 \pm 15,526 \pm 24,295 \pm 17$ and $306 \pm 17 \mathrm{nmol} \cdot \mathrm{L}^{-1}$, respectively (time of sample effect, $F=43.80 ; \quad P<0.001 ;$ partial eta ${ }^{2}=0.76$ ) (Figure 3.4). Supplementation had no affect on cortisol values (supplementation group $\mathrm{x}$ trial $\mathrm{x}$ time of sample, $P=0.437$ ). 


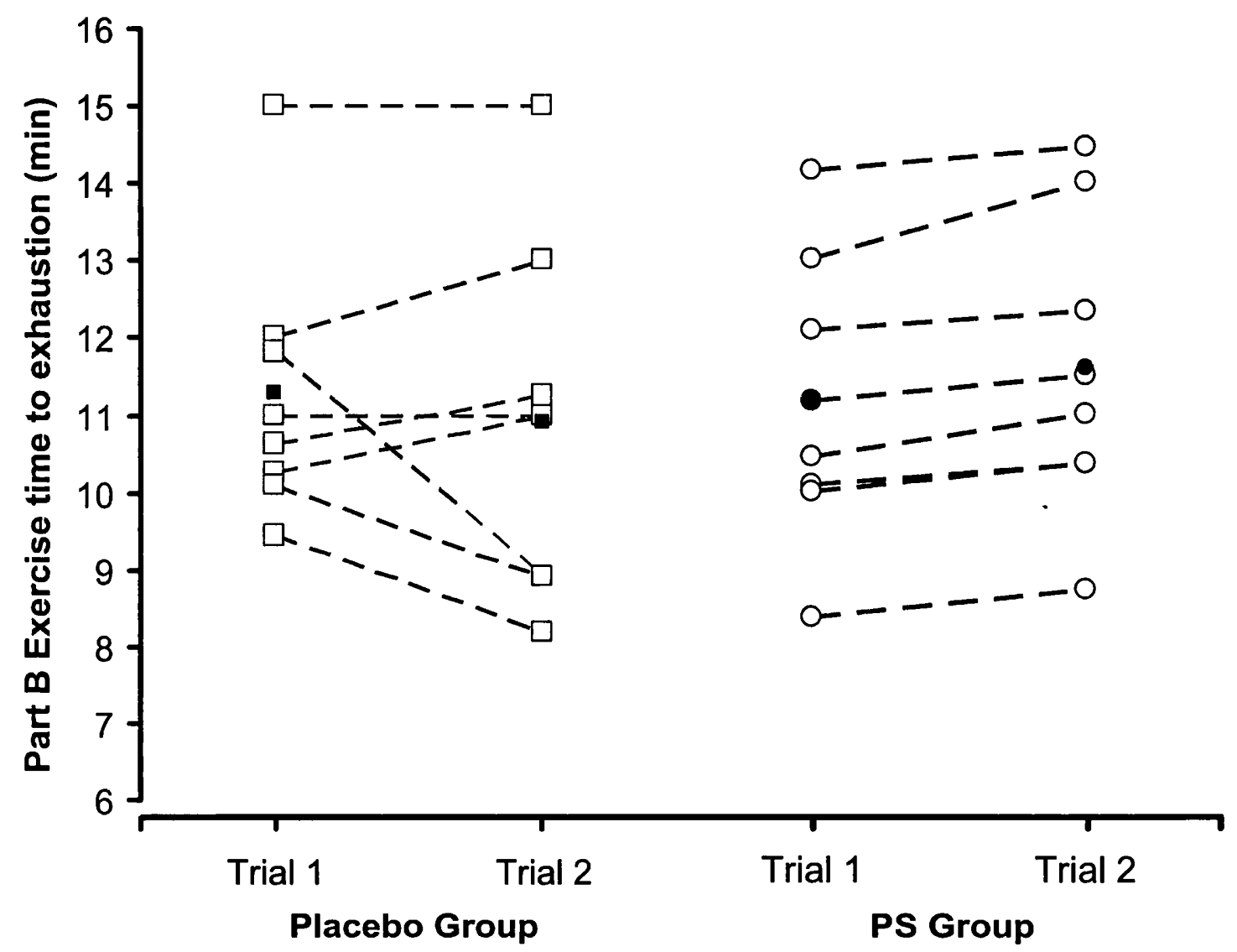

Figure 3.2 Performance during the multistage fitness test (MSFT) undertaken during Part B of each exercise trial. Individual data is presented as open shapes and filled shapes present mean group values. Trial 1: pre-supplementation; Trial 2: postsupplementation. 


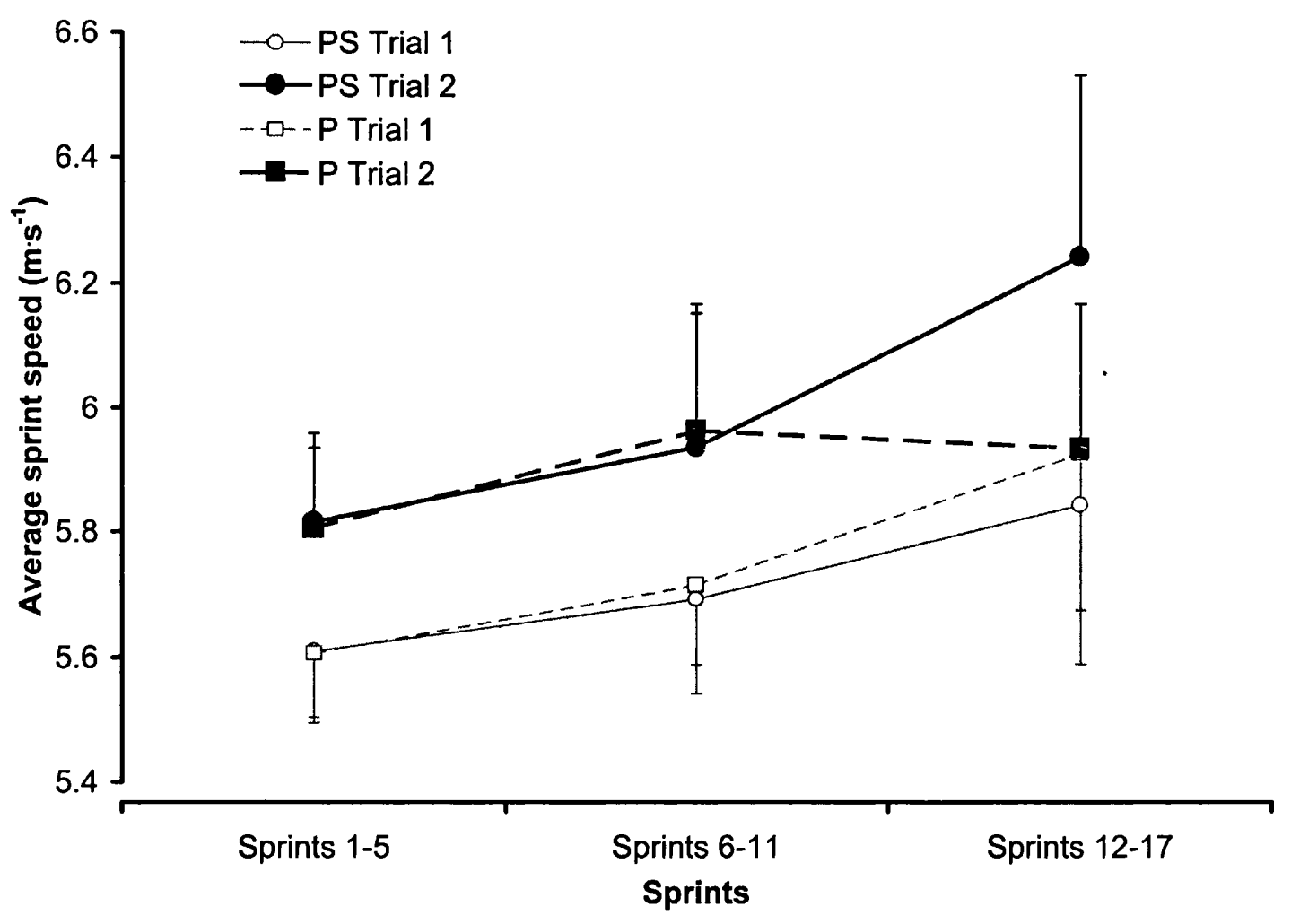

Figure 3.3 Sprinting speeds during Part A of the exercise protocol. Values represent mean $\pm \mathrm{SEM}$ (supplementation group $\mathrm{x}$ trial $\mathrm{x}$ time of sample, $P=0.143$; time of sample, $P=0.055)$. PS: phosphatidylserine group; $\mathrm{P}$ : placebo group. 


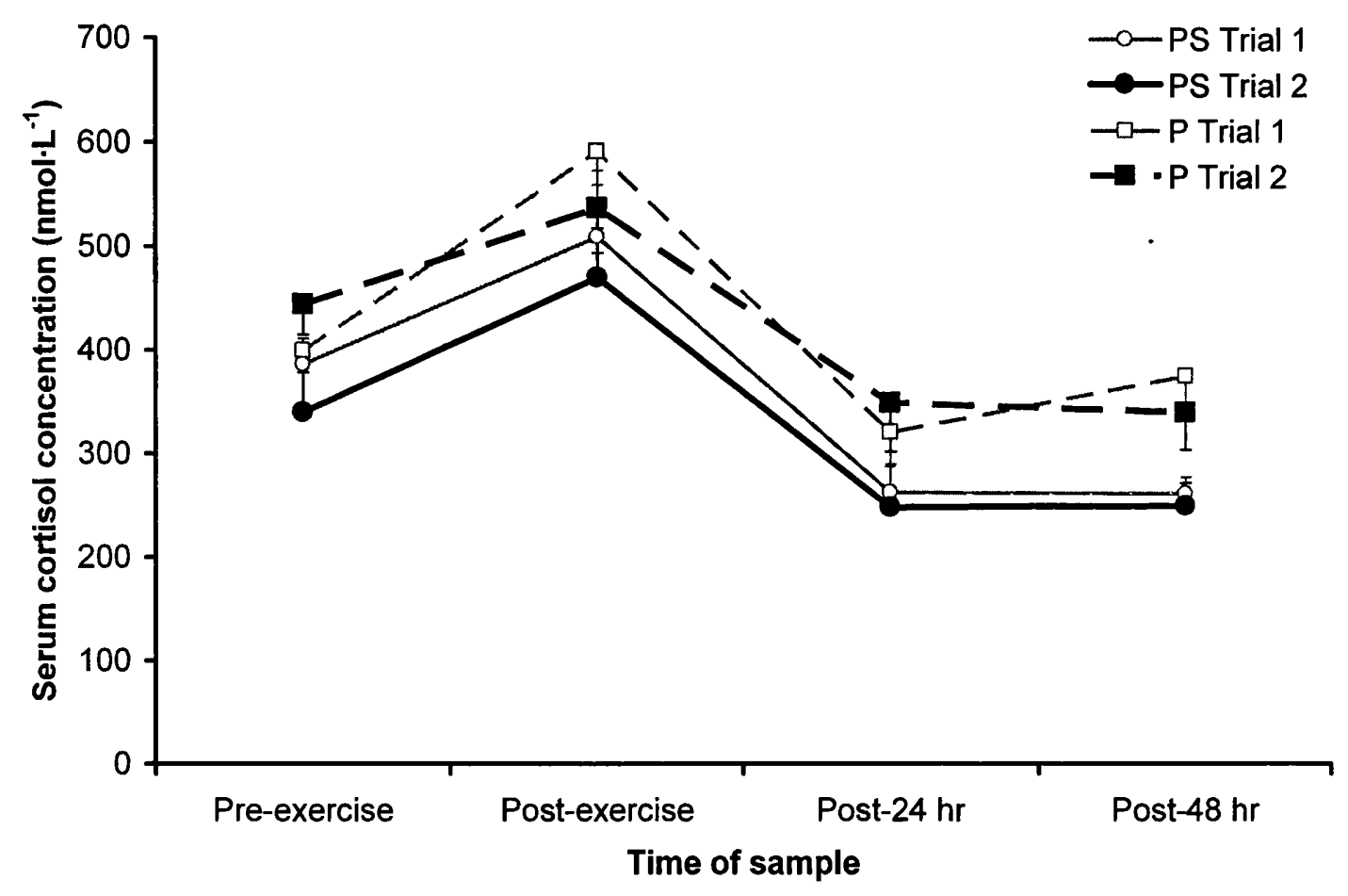

Figure 3.4 Serum cortisol concentrations. Values represent mean $\pm \mathrm{SEM}(\mathrm{N}=8$; supplementation group $\mathrm{x}$ trial $\mathrm{x}$ time of sample, $P=0.437$; time of sample, $P<0.001$ ). PS: phosphatidylserine group; P: placebo group. 
Serum $\mathrm{Mb}$ concentrations and $\mathrm{CK}$ activities are shown in Figure 3.5. Post-exercise $\mathrm{Mb}$ and CK were above Pre-exercise values in all trials, being elevated by $296 \pm 48$ and $76 \pm 7 \%$, respectively (time of sample effects being: $F=42.65 ; P<0.001 ;$ partial et $a^{2}=0.75$ and $F=16.59 ; P<0.001 ;$ partial $e t a^{2}=0.54$, respectively). The activity of CK peaked at Post-24 h in all trials, while Mb values had returned to Pre-exercise values at Post-24 h. No significant interaction effects were determined for $\mathrm{Mb}$ and CK (supplementation group $\mathrm{x}$ trial $\mathrm{x}$ time of sample being $P=0.190$ and $P=0.596$, respectively). General soreness was elevated the day following exercise (Post-24 h) above Pre-exercise values and then returned to baseline values at Post-48 h (Table 3.4). The pattern of soreness was not significantly influenced by supplementation.

Serum Hydroperoxide Concentrations and Conjugated Diene Lag Times

Serum HPO concentrations were significantly elevated at Post-exercise by approximately $24 \pm 5 \%$ (time of sample effect, $F=9.31 ; P=0.001 ;$ partial $^{\text {eta }}{ }^{2}=0.40$ ) in all trials (Figure 3.6). Supplementation had no significant effect on HPO (supplementation group $\mathrm{x}$ trial $\mathrm{x}$ time of sample, $P=0.500$ ). Exercise caused a significant elevation in conjugated diene lag times at Post-exercise (time of sample effect, $F=4.20 ; P=0.011 ;$ partial eta $^{2}=0.23$ ), being $67.5 \pm 1.4,69.8 \pm 1.1,68.4 \pm 1.0$ and $66.5 \pm 1.2 \mathrm{~min}$ at Pre-exercise, Post-exercise, Post-24 h and Post-48 h, respectively (Figure 3.7). Supplementation did not affect conjugated diene lag times (supplementation group $\mathrm{x}$ trial $\mathrm{x}$ time of sample, $P=0.489$ ). 


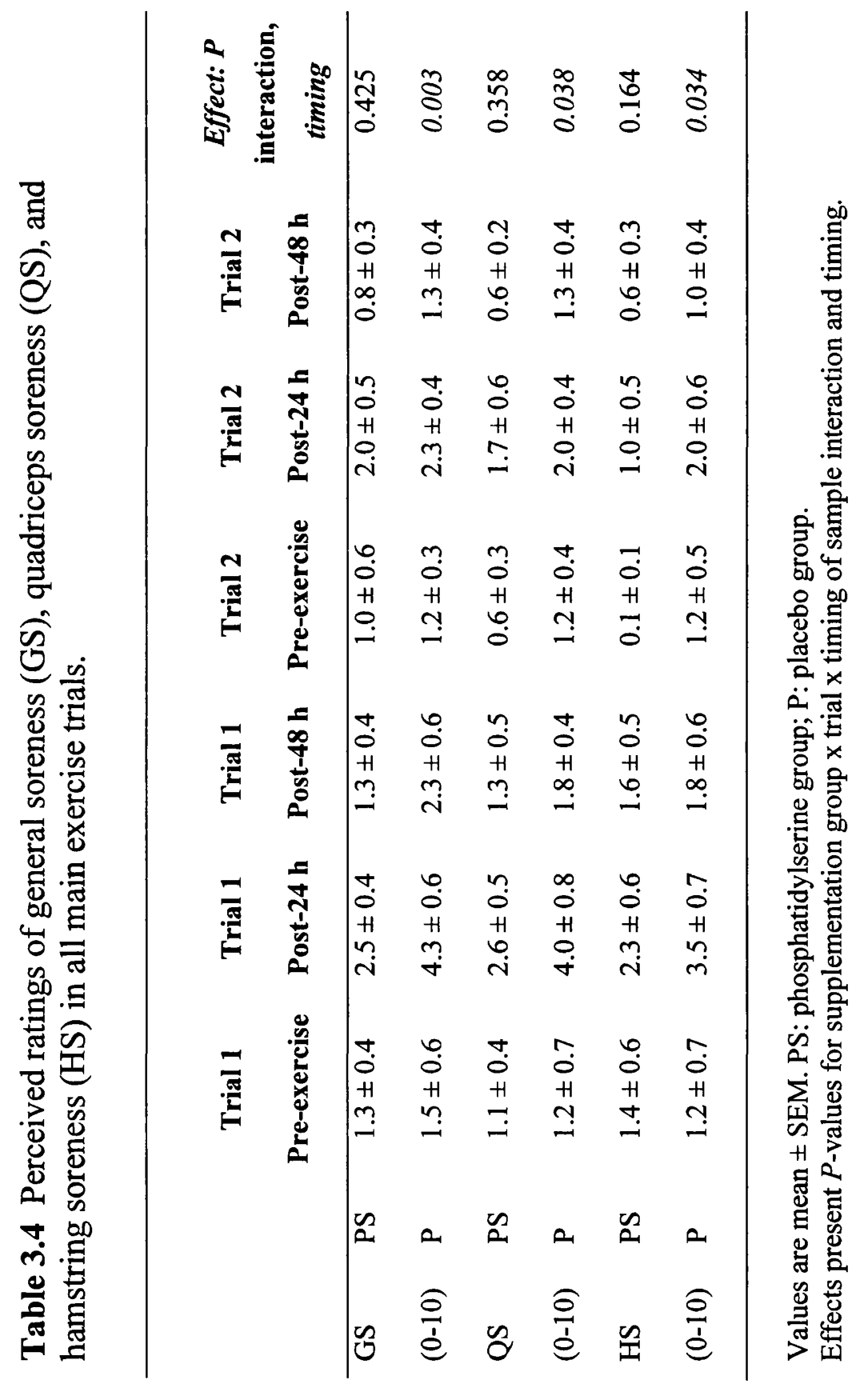



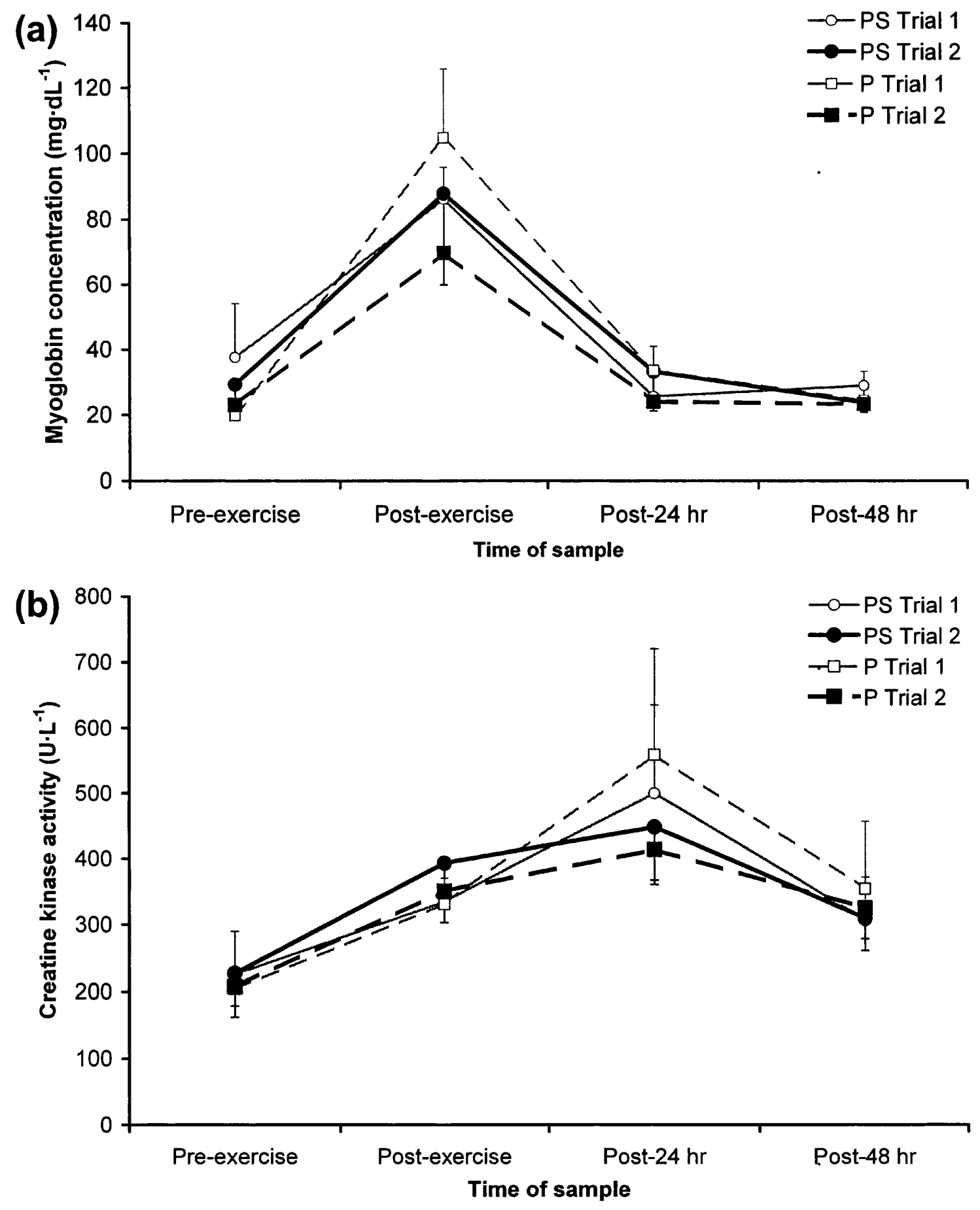

Figure 3.5 (a) Serum myoglobin concentrations and (b) creatine kinase activities. Values represent mean $\pm \mathrm{SEM}(\mathrm{N}=8$; supplementation group $\mathrm{x}$ trial $\mathrm{x}$ time of sample, $P=0.190$ and $\mathrm{P}=0.596$, respectively; time of sample, $P<0.001$ and $P<0.001$, respectively). PS: phosphatidylserine group; P: placebo group. 


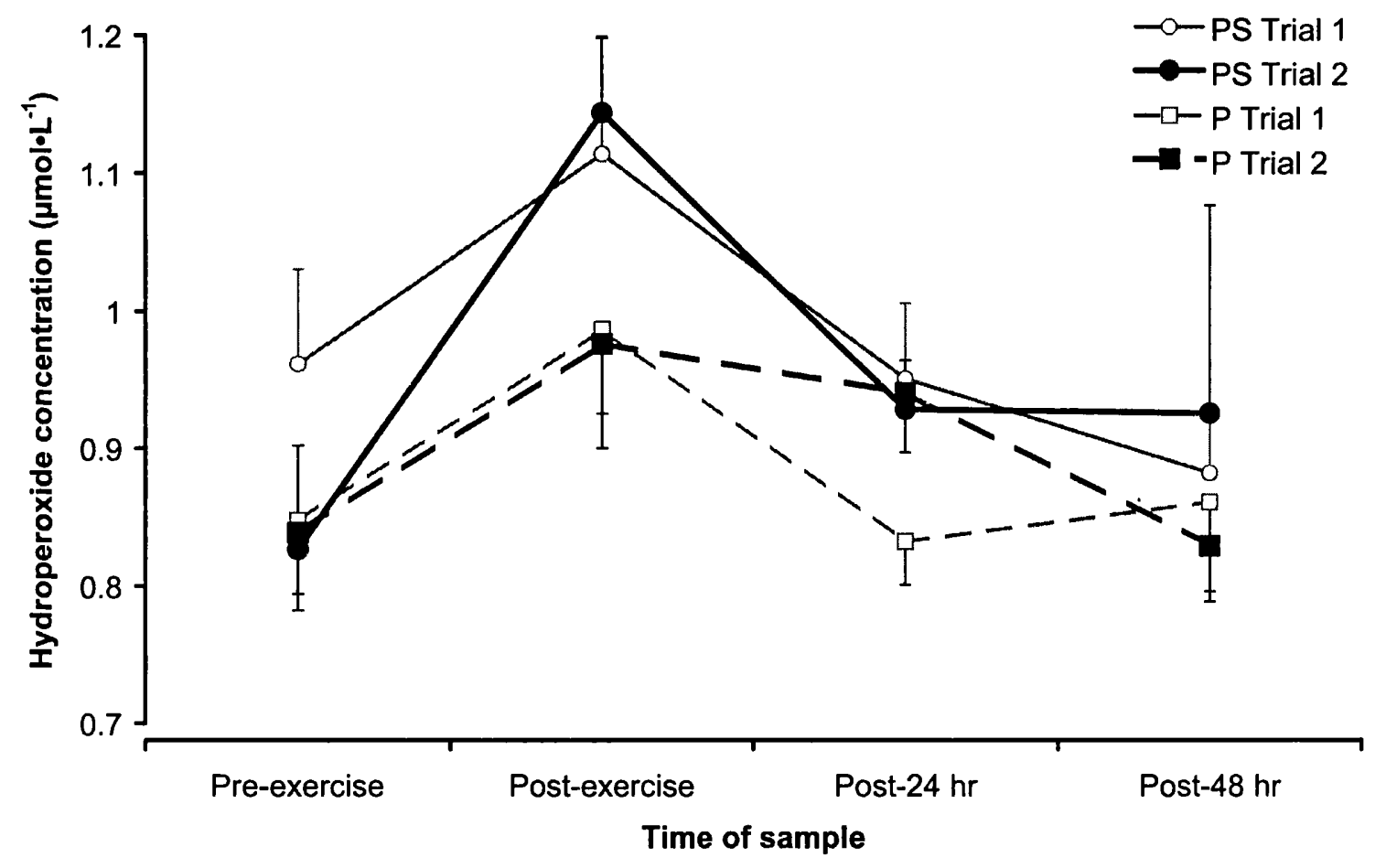

Figure 3.6 Serum hydroperoxide concentrations. Values represent mean \pm SEM $(\mathrm{N}=8$; supplementation group $\mathrm{x}$ trial $\mathrm{x}$ time of sample, $P=0.500$; time of sample, $P=0.001)$. PS: phosphatidylserine group; $\mathrm{P}$ : placebo group. 


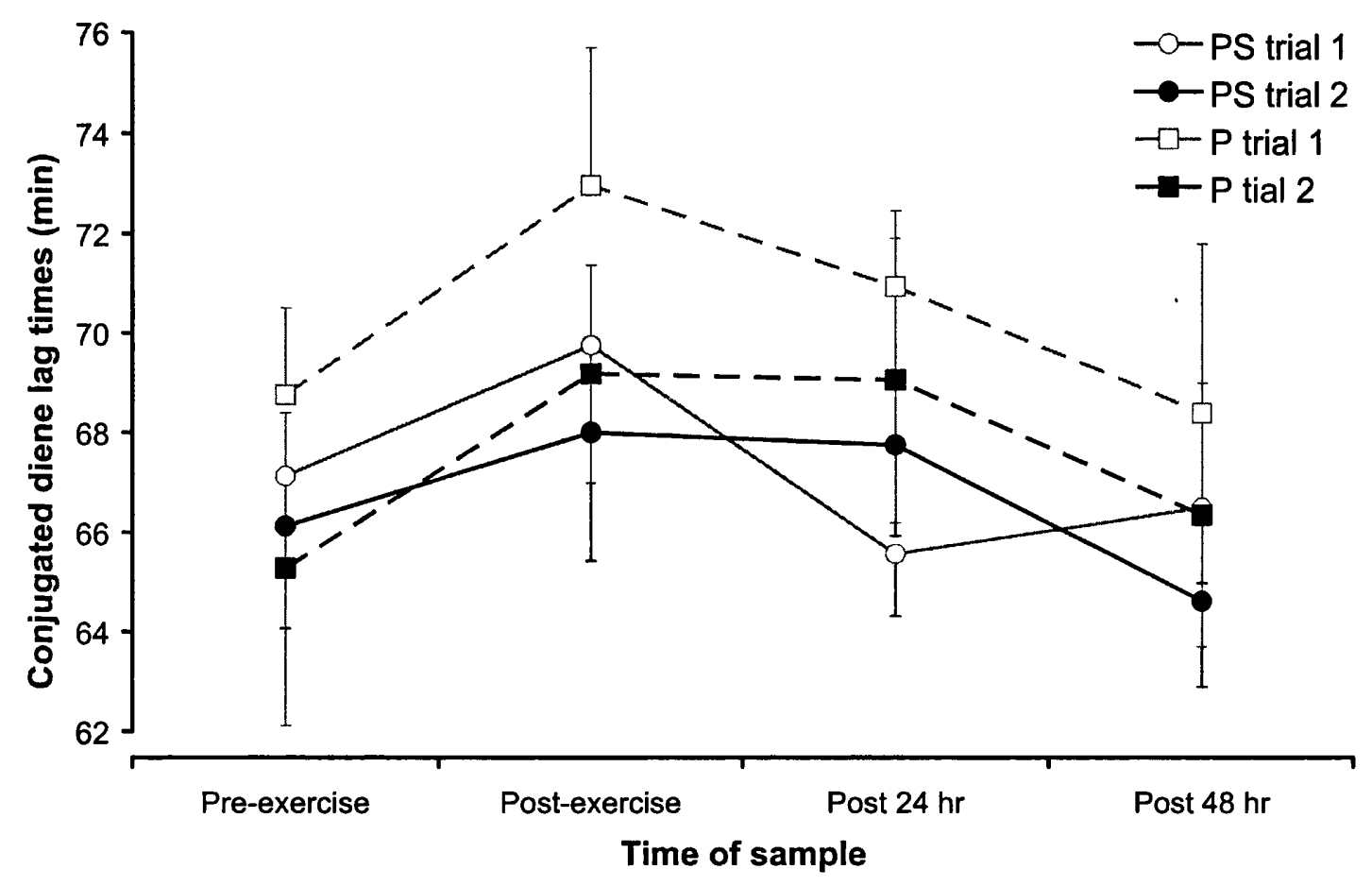

Figure 3.7 Conjugated diene lag times. Values represent mean $\pm \mathrm{SEM}(\mathrm{N}=8$; supplementation group $\mathrm{x}$ trial $\mathrm{x}$ time of sample, $\mathrm{P}=0.489$; time of sample, $\mathrm{P}=0.011$ ). PS: phosphatidylserine group; $P$ : placebo group. 
Post-exercise plasma vitamin C concentrations were significantly elevated when compared with Pre-exercise values in all trials (time of sample effect, $P=0.001$ ), although these values were similar before and after supplementation in PS and P (Table 3.5). Plasma concentrations of $\alpha$-tocopherol, retinol and $\beta$-carotene were similar throughout the duration of each trial and were not significantly different between trials (Table 3.5). Supplementation significantly affected plasma $\gamma$-tocopherol concentrations (supplementation group $\mathrm{x}$ trial $\mathrm{x}$ time of sample, $F=4.52 ; P=0.008$; partial eta $\left.^{2}=0.24\right)$. Following supplementation the change in Preexercise and Post-exercise values were $75 \pm 21$ and $94 \pm 22 \%$ higher than $\mathrm{T} 1$ values, respectively in PS (Table 3.5). 


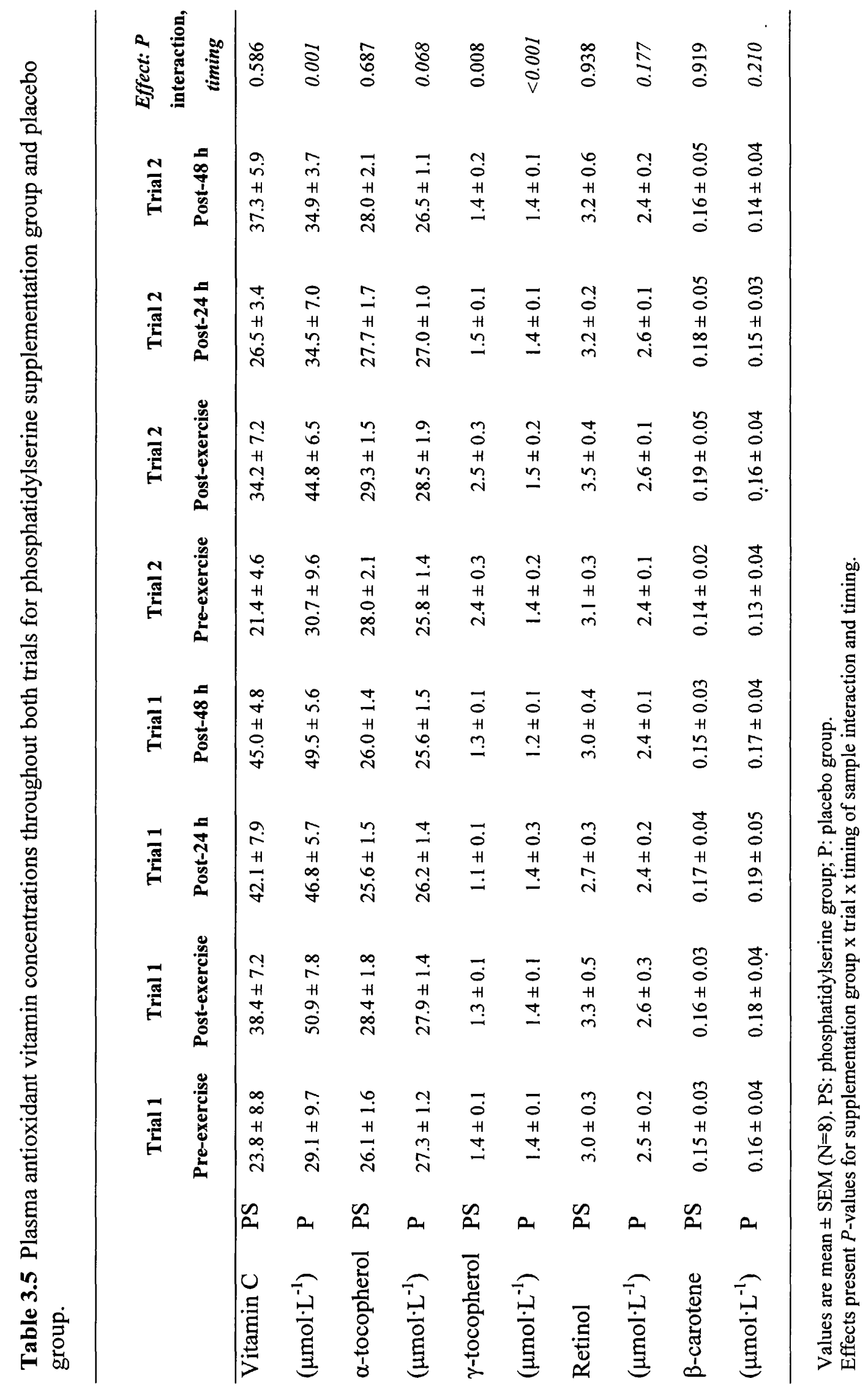




\subsection{DISCUSSION}

The study primarily aimed to investigate whether short-term S-PtdSer supplementation would influence muscle soreness and markers of muscle damage and oxidative stress following an exhaustive running protocol consisting of intermittent running designed to simulate the physiological demands of soccer that was immediately followed by a progressive run in well-accustomed university soccer players. The supplementation regime significantly increased plasma $\gamma$ tocopherol concentrations throughout the day of exercise; but did not cause increases in the concentrations of any other antioxidant vitamin measured. Moreover, supplementation with S-PtdSer had no effect on perceived muscle soreness or markers of muscle damage and lipid peroxidation. An unexpected and potentially useful outcome from this investigation was that oral supplementation with $750 \mathrm{mg} \cdot \mathrm{d}^{-}$ ${ }^{1} \mathrm{~S}$-PtdSer for $10 \mathrm{~d}$ tended to improve running performance.

The uptake and biokinetics of PtdSer following oral administration in humans are largely unknown. However, oral administration with PtdSer in rats suggests that although the majority of the exogenous phospholipid content is hydrolysed and degraded, small fractions of PtdSer remain available (Pepeu et al., 1996). Furthermore, the addition of exogenous PtdSer to mammalian cell cultures results in efficient incorporation within a variety of cell membranes (Nishijima et al., 1986). Therefore, it is likely that the short-term supplementation regime used in the current study will have caused transient increases in the PtdSer content within systemic circulation and incorporation of PtdSer within cell membranes in the PS group. 
In the present study, exercise caused rises in serum cortisol concentrations that were similar to those previously reported following comparable activities (Thompson et al., 2001a), suggesting that the protocol activated the hypothalamo-pituitary-adrenal (HPA) axis (Monteleone et al., 1990). However, supplementation had no effect on serum cortisol concentration at any time during the investigation. This finding does not support those of Monteleone et al. (1990; 1992) who reported that PtdSer supplementation resulted in significant reductions in plasma adrenocorticotrophic hormone $(\mathrm{ACTH})$ and cortisol concentrations during submaximal exercise in untrained subjects.

The dose of S-PtdSer used in the current study was similar to the regime employed by Monteleone et al. (1992) that used $800 \mathrm{mg} \cdot \mathrm{d}^{-1}$, but the possibility exists that this dose might not have been high enough to attenuate the cortisol response in these active individuals. Alternatively, increased blood cortisol concentration is a generic response to stress from both psychological and physical origin; consequently, as the current experimental design was completed until volitional exhaustion during both trials, the overall stress might have been similar during all trials. It remains plausible, although speculative, that exogenous S-PtdSer might have influenced membrane-mediated factors that might have attenuated the stimulation of ACTH and subsequent cortisol release (Monteleone et al., 1992) at some time point during exercise (although serial blood samples were not available during the exercise protocol to evaluate this). Thus, a blunted activation of the HPA axis might have led to a reduced perception of stress and partially contributed to the finding that SPtdSer supplementation tended to enhance running performance in PS when compared with P. Also, increases in membrane-bound PtdSer might have the 
potential to enhance muscle excitation-contraction coupling, potentially through the activation of different protein kinase $\mathrm{C}$ isoforms (Kajikawa et al., 1983) and/or enhanced calcium uptake (Pepeu et al., 1996), which might have contributed to the finding that supplementation tended to improve exercise performance.

The exercise induced increases in perceived soreness and serum concentration of $\mathrm{Mb}$ and the activity of CK were comparable to those previously reported for accustomed individuals that completed similar activity patterns (Thompson et al., 2001). Nevertheless, supplementation had no effect on markers of muscle damage (CK and $\mathrm{Mb}$ ) or the extent of perceived soreness in PS or P. These findings are in spite of a tendency for the PS group to increase exercise performance during Part B of the protocol. Exercise elevated serum hydroperoxide concentrations and conjugated diene lag times to a similar extent before and following supplementation, demonstrating that exercise caused radical production that overwhelmed antioxidant defence and led to comparable lipid peroxidation during all trials. Therefore, it is possible that S-PtdSer might have had some protective effect in reducing muscle damage and inflammation that was masked by the unexpected tendency for performance to be improved in the PS group. Further protection might have been afforded against the delayed onset of muscle damage that has been recently associated with inflammatory processes caused by free-radicals (Aoi et al., 2004), had supplementation been continued on the days following exercise.

Supplementation in PS led to approximately 2 -fold increases in plasma $\gamma$-tocopherol concentrations before and following exercise; although, concentrations returned to basal levels Post-24 h. Oral supplementation with foods containing high 
concentrations of $\gamma$-tocopherol have been demonstrated to elevate blood $\gamma$-tocopherol concentrations; however, the mechanism for the increases in plasma $\gamma$-tocopherol concentrations following S-PtdSer supplementation are unclear, since soy oil consumption has previously been shown to have no effect on blood $\gamma$-tocopherol concentrations in humans (Cooney et al., 2001). Nonetheless, increases in plasma and tissue $\gamma$-tocopherol in rats have been demonstrated to attenuate protein and ascorbate oxidation following inflammation-mediated damage without affecting $\alpha$-tocopherol concentrations (Jiang et al., 2002). Thus, the transient increases in plasma $\gamma$-tocopherol concentrations and probable increases in tissue $\gamma$-tocopherol concentrations might have augmented the antioxidant defence in PS during T2, although supplementation had no effect on concentrations of the other plasma antioxidant vitamins (vitamin $\mathrm{C}, \alpha$-tocopherol, retinol and $\beta$-carotene) or lipid peroxidation. In addition, evidence has been presented suggesting anti-inflammatory effects of $\gamma$-tocopherol that are different from its free radical scavenging actions (Jiang and Ames, 2003) possibly mediated through specific protein binding interactions (Hensley et al., 2003).

In conclusion, S-PtdSer supplementation had no effect on perceived soreness and markers of muscle damage and oxidative stress caused by exhaustive exercise designed to simulate the movement patterns of soccer. There are several plausible reasons for the lack of effect of S-PtdSer supplementation in the current study. The supplementation regime used in the current study might not have been optimal in attenuating the activation of the HPA axis, oxidative stress and inflammation responses to exhaustive exercise. In addition, as the exercise was continued to exhaustion, any effect of supplementation might have been shrouded by differing 
amounts of external work. The tendency for PS to improve running performance when compared with $P$ suggested that there might be a true effect of PtdSer supplementation; therefore, the potential ergogenic effects of S-PtdSer warrant future investigation. 


\section{CHAPTER FOUR}

\section{EXPERIMENTAL STUDY TWO}

Phosphatidylserine supplementation and recovery

following downhill running 


\subsection{INTRODUCTION}

Phosphatidylserine (PtdSer) is a phospholipid species that is incorporated in the membrane of eukaryotic cells and exhibits a range of important regulatory functions within mammalian cells (Blokland et al., 1999). The addition of PtdSer to mammalian tissues inhibits the production of pro-inflammatory cytokines (Gilbreath et al., 1986; Aramaki et al., 1997) and induces anti-inflammatory responses (Huynh et al., 2002) in in-vitro preparations. In addition, intravenously injected PtdSer has been demonstrated to have an inhibitory effect on the immune response to endotoxin-induced inflammation in rodents (Monastra and Bruni, 1992). Although the mechanism(s) by which PtdSer inhibits the immune response remain unclear, recent data suggest that PtdSer inhibits immune responses by acting on cell types (e.g., macrophages, fibroblasts, neutrophils, endothelial cells, epithelial cells) at the site of inflammation (Hoffmann et al., 2005). Furthermore, in-vitro studies have demonstrated that PtdSer has the potential to act as an antioxidant with the ability to protect cells against oxygen-derived free radicals (Latorraca et al., 1993) and suppress iron-dependent lipid peroxidation (Dacaranhe and Terao, 2001).

Soybean-derived PtdSer (S-PtdSer) has been established as a safe oral supplement for human consumption, using established biochemical and haematological parameters (Jorissen et al., 2002); additionally, no side effects following various supplementation regimes have been reported (Pepeu et al., 1996). Fahey and Pearl (1998) reported that short-term oral S-PtdSer attenuated the perception of muscle soreness that followed intense weight training. Although the mechanism(s) responsible for this action were unclear, these authors suggested that reductions in 
circulatory cortisol concentrations following exercise might have been associated with decreased protein degradation. The aforementioned anti-inflammatory and antioxidant properties of PtdSer led to experimental study 1, where the effect of S-PtdSer supplementation on the magnitude of lipid peroxidation following exhaustive intermittent running was investigated. Changes in serum HPO concentrations suggested that this exercise elevated lipid peroxidation to an equal extent before and following $750 \mathrm{mg} \cdot \mathrm{d}^{-1} \mathrm{~S}$-PtdSer for $10 \mathrm{~d}$. However, exercise performance tended to be improved following S-PtdSer supplementation and since the blood samples were taken following the completion of maximal exercise, free radical production was probably higher following S-PtdSer supplementation. Consequently, it was plausible that the potential antioxidant properties of PtdSer might have been concealed in this study. In addition, supplementation was ceased on the day of exercise and it is possible that continuing S-PtdSer supplementation during the early stages of an ensuing acute inflammatory response, might afford greater protection.

Eccentric muscle activity causes muscle injury and leads to an acute inflammatory response (Childs et al., 2001). During this response, neutrophils and macrophages migrate to the location of muscle damage, penetrate the damaged tissue, elevate free radical production and activate cytokines (Macintyre et al., 1995). Additionally, exercise produces increases in mitochondrial oxygen flux that have been implicated as a source of free radicals during the reduction of molecular oxygen in the electron transport chain (Dekkers et al., 1996). Free radicals can lead to oxidative damage in a wide range of molecular structures including lipids, proteins and DNA, where the antioxidant defences are overwhelmed (Kanter et al., 1988). Therefore, downhill 
treadmill running has the potential to initiate inflammation and increase oxidative stress. These processes have the potential to cause muscle damage, the symptoms of which include: soreness, decrease in the range of motion of joints in affected limbs, decreased muscular strength, decreased power output, leakage of myofibre proteins, inflammation and oedema (Kendall and Eston, 2002).

The purpose of the current study was to investigate the antioxidant and antiinflammatory effects of short-term S-PtdSer supplementation on markers of muscle damage, inflammation and oxidative stress following prolonged downhill running.

\section{Null Hypotheses}

$\mathrm{HO}_{1}$ : Downhill running will not influence markers of antioxidant status, tissue oxidation, muscle damage, inflammation, perceived feeling states, and muscle soreness.

$\mathrm{HO}_{2}$ : Supplementation with S-PtdSer will not influence markers of inflammation, antioxidant status, lipid peroxidation, muscle damage, and muscle soreness after downhill running.

$\mathrm{HO}_{3}$ : Supplementation with S-PtdSer will not influence neuroendocrine responses to downhill running.

$\mathrm{HO}_{4}$ : Supplementation with S-PtdSer will not alter perceived feeling states during downhill running. 


\subsection{METHODS}

\section{Subjects}

Ten healthy males volunteered to undertake this study; however, eight subjects (age: $21.0 \pm 0.3$ years; height: $1.79 \pm 0.02 \mathrm{~m}$; body mass: $81.2 \pm 3.2 \mathrm{~kg}$ ) completed all of the study requirements and were included in the subsequent analyses. All subjects were informed about the potential risks of the study and gave written informed consent for their participation in the study, which was approved by a university ethics committee and completed in accordance with the policy statement of the American College of Sports Medicine. No subject had prior history of cardiovascular or respiratory disease and all subjects were non-smokers. Potential subjects attended an interview prior to undertaking the study and were subsequently excluded if they had taken nutritional supplements in the last 8 weeks.

\section{Experimental Design}

Over a period of approximately eleven weeks, each subject completed five downhill runs on a motorised treadmill. Approximately $14 \mathrm{~d}$ after an initial familiarisation trial the subjects completed four main trials (trials 1-4). Trials 1 and 3 provided presupplementation control trials as the washout kinetics of PtdSer are currently unknown. Following trials 1 and 3 the subjects were assigned, in a double-blind and

balanced order crossover fashion, to receive either $750 \mathrm{mg} \cdot \mathrm{d}^{-1} \mathrm{~S}$-PtdSer (PS) or a weight matched glucose polymer placebo (P). Supplementation was taken for a 10-d 
period that began $7 \mathrm{~d}$ prior to Trials 2 and 4 . The duration between trials 1 and 2 and trials 3 and 4 was exactly 14 d. Trials 2 and 3 were separated by a 4 -week 'washout' period. Venous blood, measurements of joint range of motion, and ratings of perceived soreness and feelings were assessed prior to exercise (Pre-exercise), after exercise (Post-exercise) the day following exercise (Post-24 h) and the second day after exercise (Post-48 h) during each Trial. The experimental design is illustrated in Figure 4.1.

The S-PtdSer supplements were manufactured using the method of specific transesterification of soybean lecithin and then blended with additional soybean lecithin to provide a concentration of $20 \%$ PtdSer (Lucas Meyer; Hamburg, Germany). Both supplements were administered in capsules and placed in generic packaging. Subjects were instructed to maintain their normal diet and activity patterns throughout the study. Subjects weighed and recorded the food that they consumed for two days prior to and for three days after exercise. Weighed food records were analysed using commercial software (CompEat v5.8.0; Nutrition Systems, UK). In addition, the subjects were instructed to abstain from strenuous exercise for three days prior to and two days following exercise. At the completion of the study all subjects gave their verbal assurance that they had complied with all instructions. 


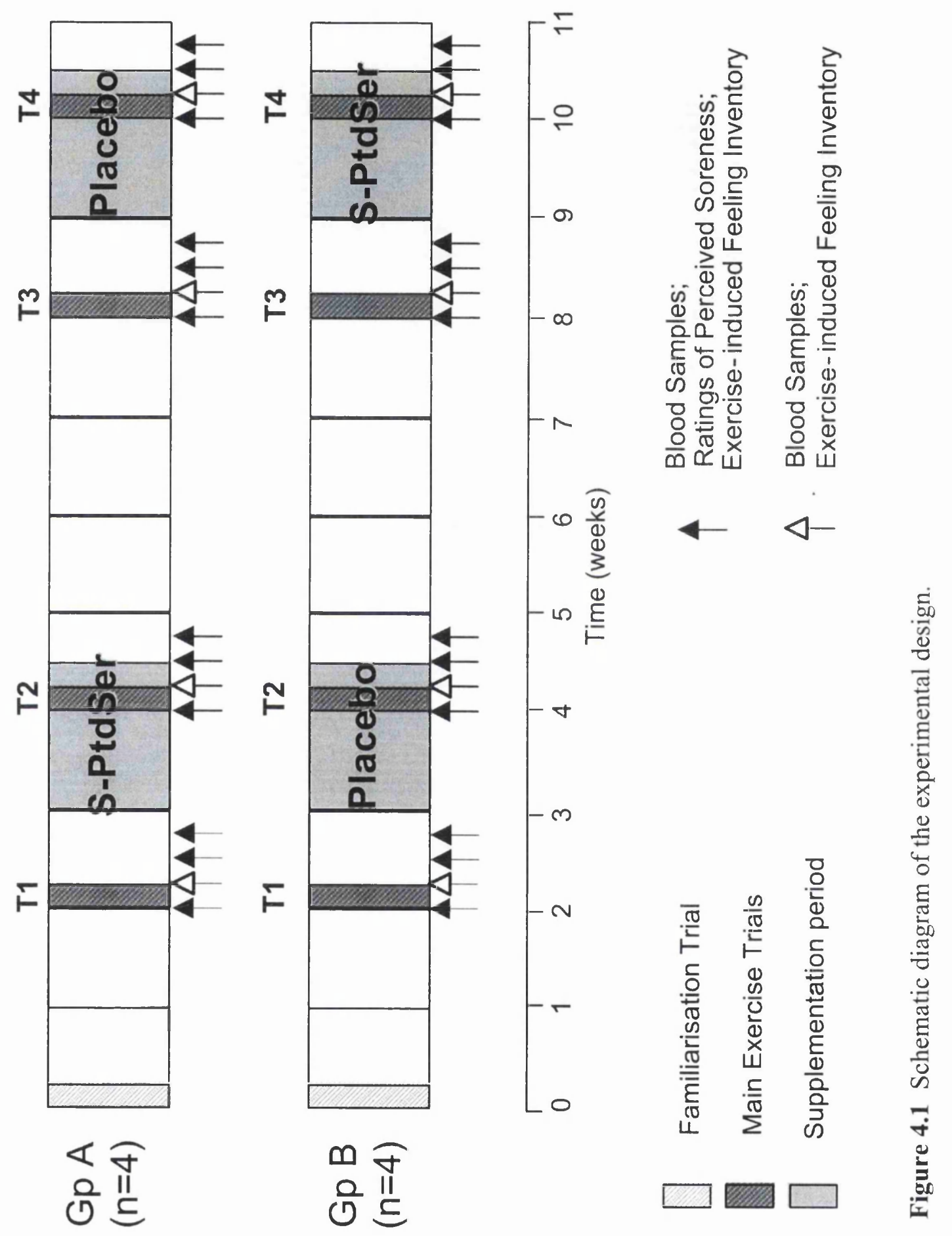




\section{Preliminary Procedures}

The familiarisation exercise trial served to reduce the anticipated trial order effects that are apparent following eccentric exercise protocols and to define individualised exercise intensities for trials 1 to 4 . Exercise started with a 3-min stage of horizontal running at $5 \mathrm{~km} \cdot \mathrm{h}^{-1}$. Subsequent $3-\min$ stages were undertaken at $-17.5 \%$ at a speed that began at $5 \mathrm{~km} \cdot \mathrm{h}^{-1}$ and increased at a rate of $1 \mathrm{~km} \cdot \mathrm{h}^{-1}$ until the subject reached a heart rate of $70 \%$ of age-predicted maximal heart rate. After a 3-min passive rest period each subject completed a $-17.5 \%$ treadmill run for $30 \mathrm{~min}$ at the terminal speed reached during the progressive section of the protocol. This individualised exercise protocol was recorded and repeated during each subsequent trial (trials 1 to 4).

\section{Main Trial Procedures}

On the day of each main exercise trial the subjects reported to the laboratory at approximately the same time of day $( \pm 1 \mathrm{~h})$ and following an overnight fast. Subject mass (model 712; Seca, Germany) and height (Portable Stadiometer; Holtain, UK) were recorded. The intensity of muscle soreness for the whole body general soreness (GenS), hamstrings soreness (HS), quadriceps soreness (QS) and gluteal soreness (GS) were rated on an eleven-point scale ranging from 0 (not sore) to 10 .(very, very sore) (Appendix E). The subjects completed exercise-induced feeling inventories (EFI) as described in detail by Gauvin and Rejeski (1993). Briefly, the subjects rated their feelings using the 12-item adjective scale on an analogue scale from 0 (do not 
feel) to 5 (feel very strongly) (Appendix F). The appropriate adjectives were averaged to obtain four perceived feeling states (Positive Engagement, Revitalisation, Tranquillity and Physical Exhaustion). Active range of motion (ROM) during knee flexion and hip flexion were assessed on the dominant leg with a Leighton Flexometer (Spokane, USA) using standard protocols described by Hubley-Kozey (1991). During hip flexion the subject started in a supine position on a bench with the instrument attached to the lateral side of the upper segment of the dominant leg; the dial was aligned with the greater trochanter of the femur. The dominant knee was extended and the dial was zeroed and locked while in a horizontal position on the bench. The subject was asked to move the leg in an arc upwards as far as possible with the average of 3 trials at each time point being recorded. Care was taken to confirm that the back remained in contact with the bench and that the pelvis was stabilised throughout the movement. During knee flexion the subject started in a prone position with the knees positioned at the end of the bench. The instrument was attached to the lateral side of the lower segment of the dominant leg; the dial was aligned with the lateral epicondyle and the lateral malleolus. The dominant knee was held in a neutral position and the dial.was zeroed and locked while in a horizontal position on the bench. The subject was asked to move the leg in an arc upwards as far as possible with the average of 3 trials at each time point being recorded. Care was taken to confirm that the ankle position was kept constant and that the pelvis was stabilised throughout the movement.

Following a venepuncture (Vacutainer system; Becton-Dickinson Ltd, UK), taken from an antecubital vein in the right arm, the subjects completed the eccentric exercise protocol (mean exercise time, gradient and speed were: $51.0^{\circ} \pm 1.5 \mathrm{~min}$, 
$-16.5 \pm 0.0 \%, 8.7 \pm 0.3 \mathrm{~km} \cdot \mathrm{h}^{-1}$, respectively). Heart rates (Polar S610; Polar Electro, Finland) and subjective ratings of perceived exertion (Borg, 1998) were monitored throughout the eccentric exercise protocol. Ambient temperature and humidity were recorded at the beginning and end of exercise (ETHG-912; Oregan Scientific, USA). Post-exercise EFI were obtained and venous blood samples were taken from an antecubital vein in the left arm as previously described.

Subjects returned to the laboratory on the day following (Post-24 h) and the second day following (Post-48 h) each main exercise trial at approximately the same time $( \pm$ $1 \mathrm{~h}$ ) after overnight fasts. During these visits blood samples were drawn, joint ROM were measured and ratings of soreness were completed, as previously described.

\section{Blood Sampling and Analysis}

Venous blood was collected in a 5-mL container (Becton-Dickinson Ltd, UK) containing the anticoagulant ethylenediamine tetra-acetic acid (EDTA). Several small aliquots were removed for the triplicate determination of blood lactate concentration, glucose concentration (YSI 2300, Yellow Springs Instruments, US), haemoglobin concentration (Hemocue Ltd, UK), haematocrit (Micro haematocrit MK IV, Hawksley, UK) and changes in plasma volume were estimated as previously described (Dill and Costill, 1974). The remaining blood was centrifuged at $3000 \mathrm{x} \mathrm{g}$ for $15 \mathrm{~min}$ to obtain plasma. An aliquot of plasma $(100 \mu \mathrm{L})$ was added to $900 \mu \mathrm{L}$ of freshly prepared $10 \%$ metaphosphoric acid, mixed and frozen at $-70^{\circ} \mathrm{C}$ for subsequent vitamin $\mathrm{C}$ analysis. The remaining plasma was frozen and stored at 
$-70^{\circ} \mathrm{C}$ prior to subsequent analysis. Two additional $7-\mathrm{mL}$ blood samples were collected in serum separation tubes (Becton-Dickinson Ltd, UK), left to stand for 15 min then centrifuged at $3000 \mathrm{x} \mathrm{g}$ for $15 \mathrm{~min}$ to obtain serum. The serum was transferred to appropriate containers and subsequently stored at $-70^{\circ} \mathrm{C}$ prior to analysis.

Plasma cortisol (kit EIA-1887; DRG Instuments, Germany) and adrenocorticotropic hormone (ACTH) (kit 7023; Biomerica, US) concentrations were determined in duplicate using solid-phase enzyme-linked immunosorbent assays (ELISA). Interleukin-6 (IL-6) concentrations in serum were assayed in duplicate using commercially available high sensitivity ELISA kits (Diaclone Research, France). An automated spectrophotometer (MiraS; ABX Diagnostics, UK) was used to measure plasma myoglobin $(\mathrm{Mb})$ concentrations and creatine kinase (CK) activity in duplicate with commercially available kits (A11A00165 and A11A00008, respectively; ABX Diagnostics, UK). Serum hydroperoxide (HPO) concentrations were measured using the method of Wolff (1992) as described in McEneny et al. (1998). Low density lipoprotein was isolated from plasma and oxidised according to the method of McDowell et al. (1995). Subsequently, the production of conjugated dienes was followed in duplicate at $234 \mathrm{~nm}$ (SpectraMax 190; Molecular Devices Corp, US) using the computer software SoftMax Pro Version 3.0 (Molecular Devices Corp, US); the change in absorbance (from baseline to the end of reaction) was used to quantify change in conjugated diene concentration and the time taken to reach half of the maximum oxidation ( $t_{1 / 2 \max }$ LDL oxidation) was taken as a measure of the resistance of the particle to oxidation. Vitamin $\mathrm{C}$ concentrations were determined using a fluorimetric assay using a centrifugal analyser with fluorescence 
attachment according to the method of Vuilleumier (1993). Plasma concentrations of $\alpha$-tocopherol, $\gamma$-tocopherol, retinol, $\alpha$-carotene, $\beta$-carotene and lycopene were measured by high-performance lipid chromatography with electrochemical detection according to the methods of Catignani and Bieri (1983) and Thurnham et al. (1988).

\section{Statistical Analysis}

Statistical analysis was carried out using SPSS software (version 13.0; SPSS Inc., IL, US). Group data were expressed as mean \pm SEM and statistical significance was set at the $P<0.05$ level. All data were assessed for normality (Shapiro-Wilk's test) and data that were not normally distributed $(\mathrm{Mb}$ and $\mathrm{CK})$ were log transformed prior to analyses. Data from trials 1 and 3 (pre-supplementation control trials) were analysed using mixed-model repeated measures ANOVA (within subject factors: trial x time of sample; between subject factor: group). If the $P$ value for the 3-way interaction (group $\mathrm{x}$ trial $\mathrm{x}$ time of sample) reached significance, the subject groups reacted differently and supplementation order was deemed to have had an effect. The data from all trials were analysed using repeated measures ANOVA (within subject factors: trials $\mathrm{x}$ time of sample). Mauchly's test was consulted and Greenhouse-Geisser correction was applied if the assumption of sphericity was violated. Where a significant $P$ value was identified for the main effect of trial or time (time of sample), multiple pairwise comparisons were made using Bonferonni confidence interval adjustment. Pearson product moment analysis was used to determine the strength of correlation between plasma ACTH and cortisol concentrations. 


\subsection{RESULTS}

Ambient temperature and relative humidity were similar during all trials, with mean values during all trials being $20.1 \pm 0.3^{\circ} \mathrm{C}$ and $71 \pm 3 \%$, respectively. All 3-way interactions from trial 1 and 3 were non-significant $(P \geq 0.172)$. The mean exercising heart rates ranged from $136 \pm 4$ to $137 \pm 3$ beats $\cdot \min ^{-1}$ and were similar during all trials $(P=0.951)$. Blood lactate concentrations were not different between trials $(P=0.548)$, with Post-exercise values ranging from $0.9 \pm 0.1$ to $1.1 \pm 2 \mathrm{mmol} \cdot \mathrm{L}^{-1}$ (Table 4.1). Blood glucose concentrations did not differ between trials $(P=0.057)$ or timing of sample ( $P=0.113$ ), with Post-exercise values ranging from $4.2 \pm 0.1$ to 4.4 $\pm 0.2 \mathrm{mmol} \cdot \mathrm{L}^{-1}$ (Table 4.1 ). Estimated changes in plasma volume were significantly lower at Post-exercise when compared with all other time points in the study $(\mathrm{P} \leq 0.039)$; however, no differences were observed between trials $(P=0.972)$ (Table 4.1). No differences were observed in the calculated amount or composition of the food consumed during any trial. The average daily diet comprised of $10.3 \pm 0.3$ $\mathrm{MJ} \cdot \mathrm{d}^{-1}$, of which $44 \pm 1,30 \pm 1,24 \pm 1$ and $2 \pm 1 \%$ of energy intake was obtained from carbohydrates, fats, proteins and alcohol, respectively. 


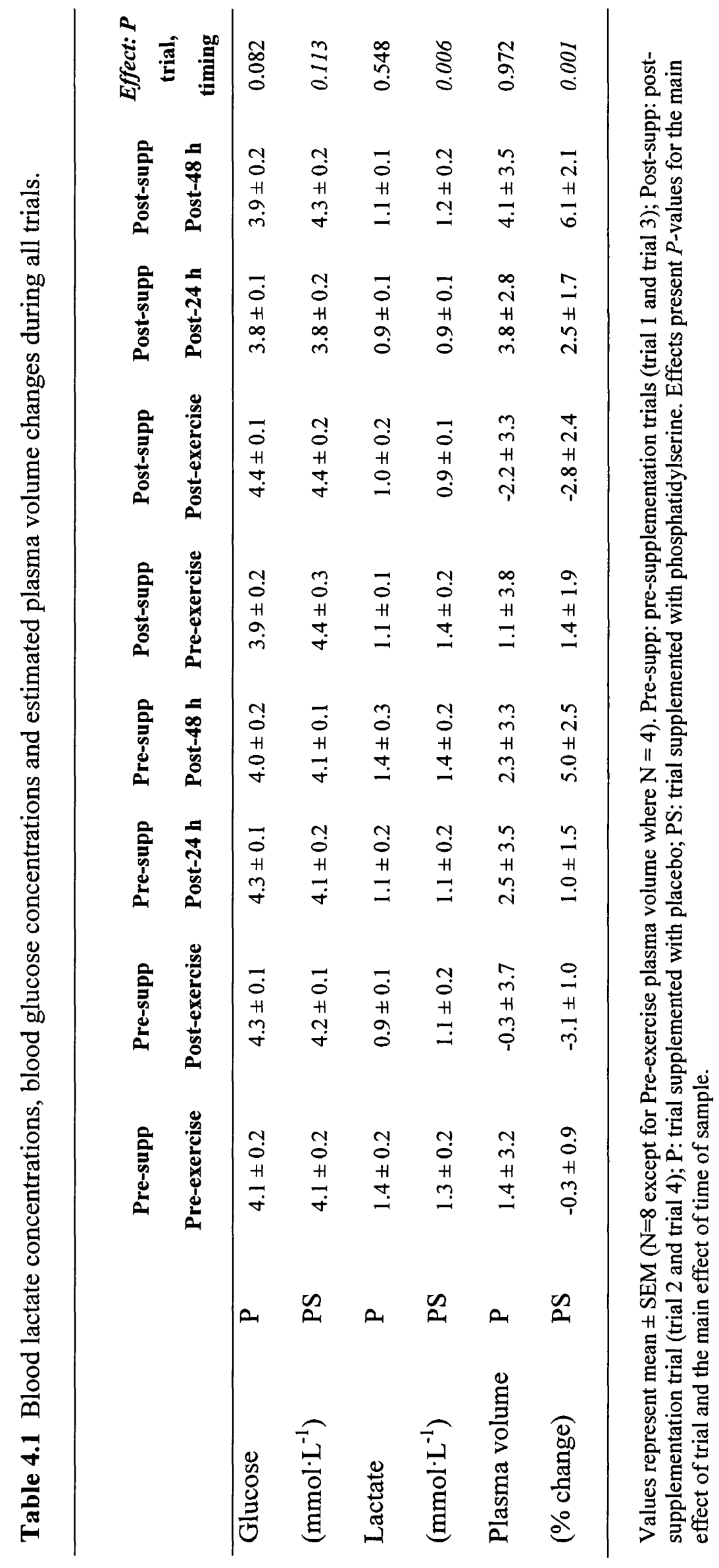


Post-exercise plasma ACTH and cortisol concentrations were $20 \pm 6$ and $27 \pm 3 \%$ below Pre-exercise values, respectively (time of sample effect, $F=4.40 ; P=0.015$; partial et $^{2}=0.80$ and $F=20.29 ; P=0.001 ;$ partial eta $^{2}=0.74$, respectively) (Figure 4.2). These values did not differ between trials (trial effect, $P=0.150$ and $P=0.166$, respectively). Plasma ACTH concentrations and plasma cortisol concentrations were significantly correlated $(\mathrm{r}=0.412 ; P<0.001)$.

Plasma Myoglobin Concentrations and Creatine Kinase Activities

Plasma Mb concentrations were elevated by $78 \pm 12 \%$ above Pre-exercise at Postexercise in all trials (time of sample effect, $F=15.50 ; P<0.001$; partial eta $^{2}=0.69$; Figure 4.3). Plasma CK activities peaked at Post-24 $\mathrm{h}$ in all trials (time of sample effect, $F=23.25 ; P<0.001$; partial eta $^{2}=0.77$; Figure 4.3 ), being $200 \pm 39 \%$ above Pre-exercise. There were no significant differences between trials for either $\mathrm{Mb}$ or CK (trial effect, $P=0.865$ and $P=0.971$, respectively). 
(a)

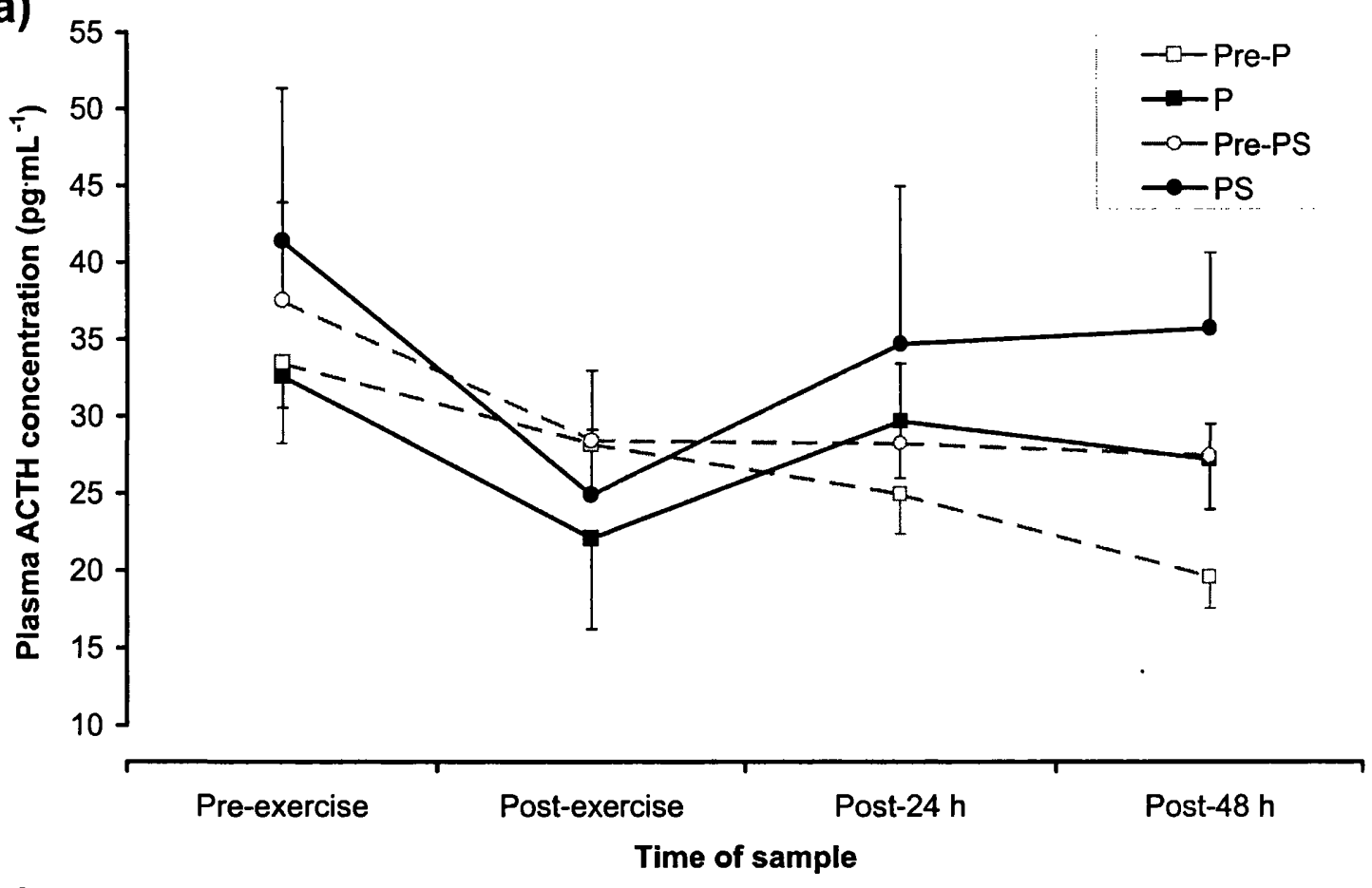

(b)

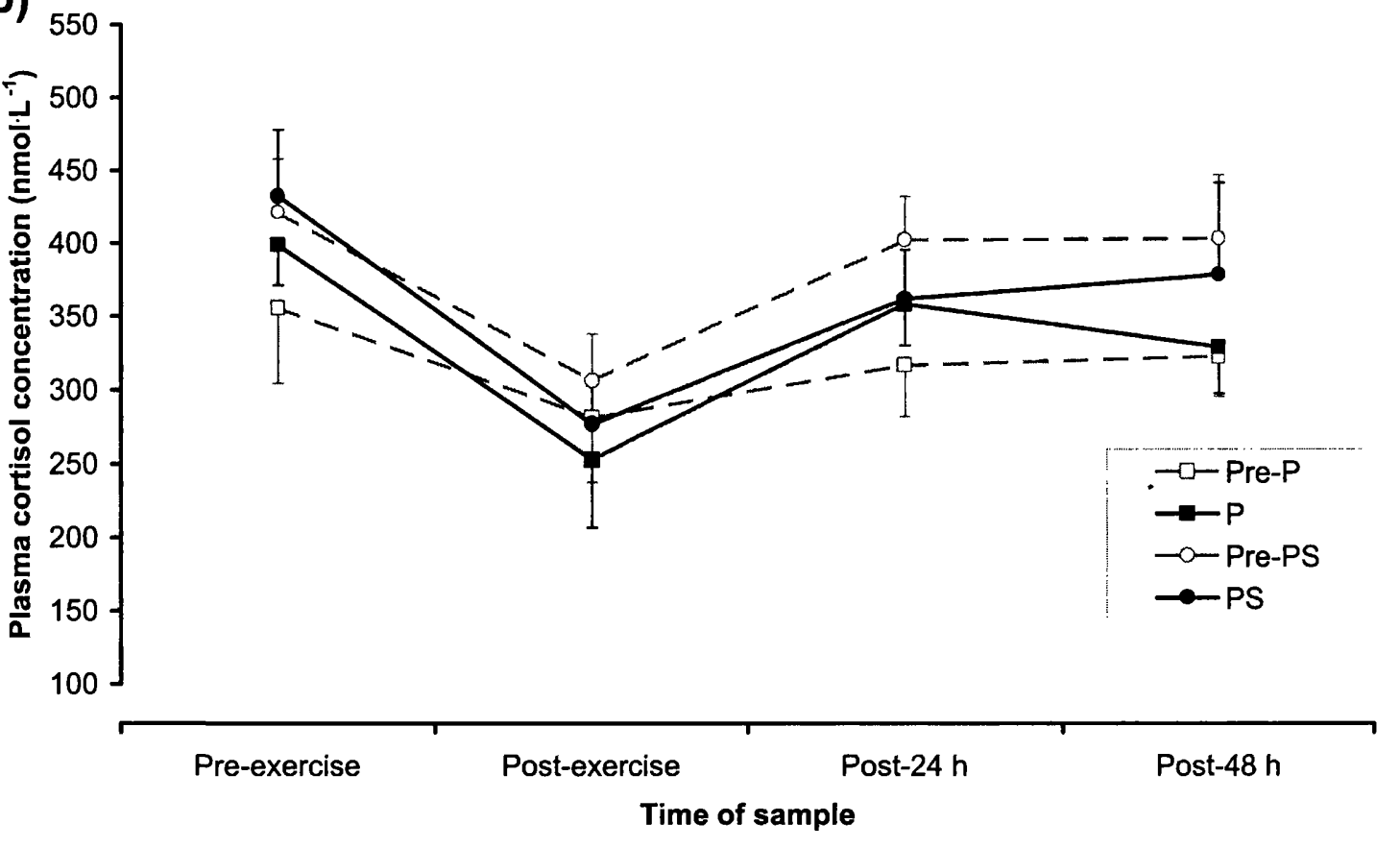

Figure 4.2 (a) Plasma adrenocorticotropic hormone (ACTH) concentrations and (b) plasma cortisol concentrations throughout each trial. Values represent mean \pm SEM (N=8). Pre-P: trial prior to supplementation with placebo; P: trial supplemented with placebo; Pre-PS: trial prior to supplementation with phosphatidylserine; PS: trial supplemented with phosphatidylserine. 
(a)

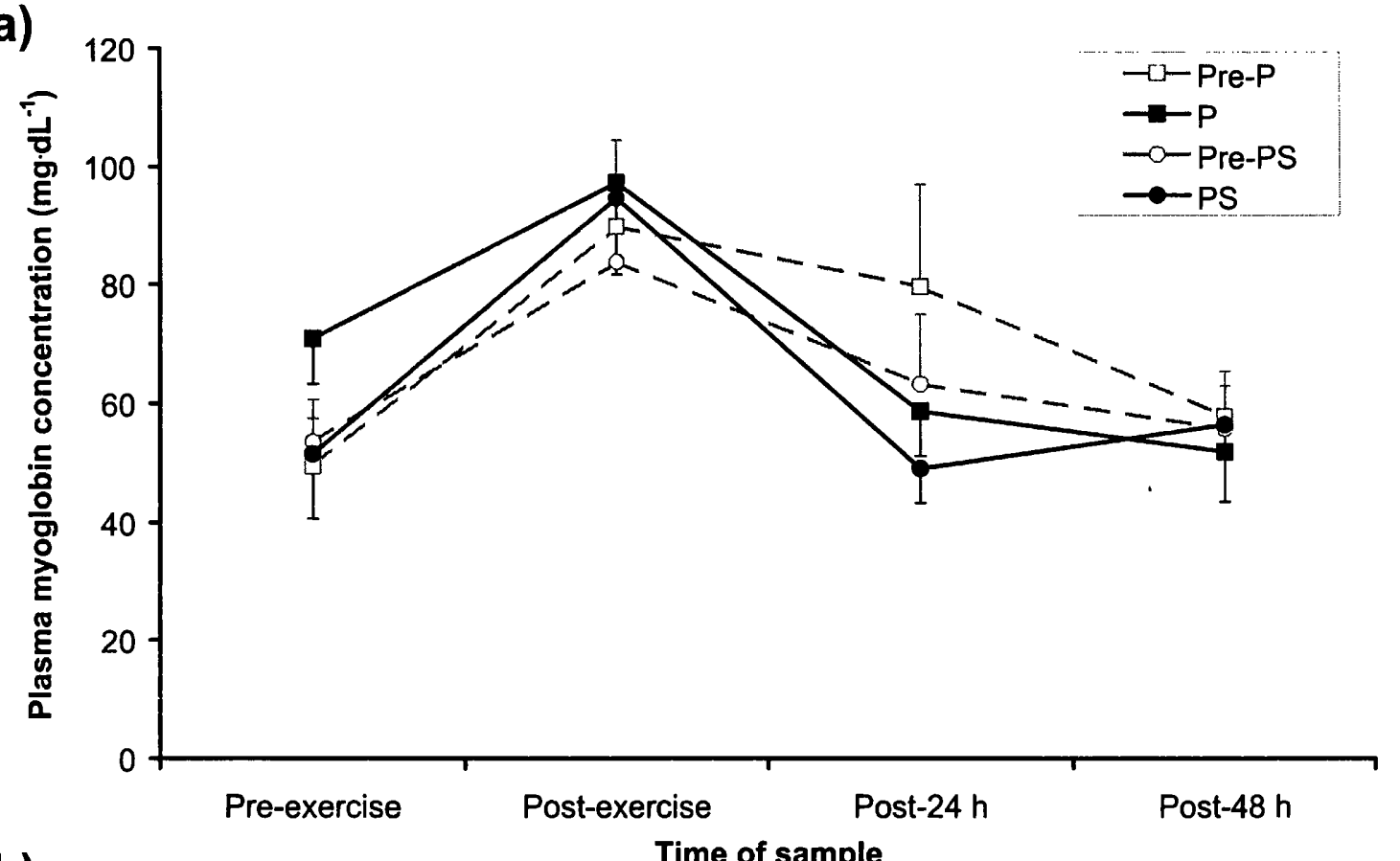

(b)

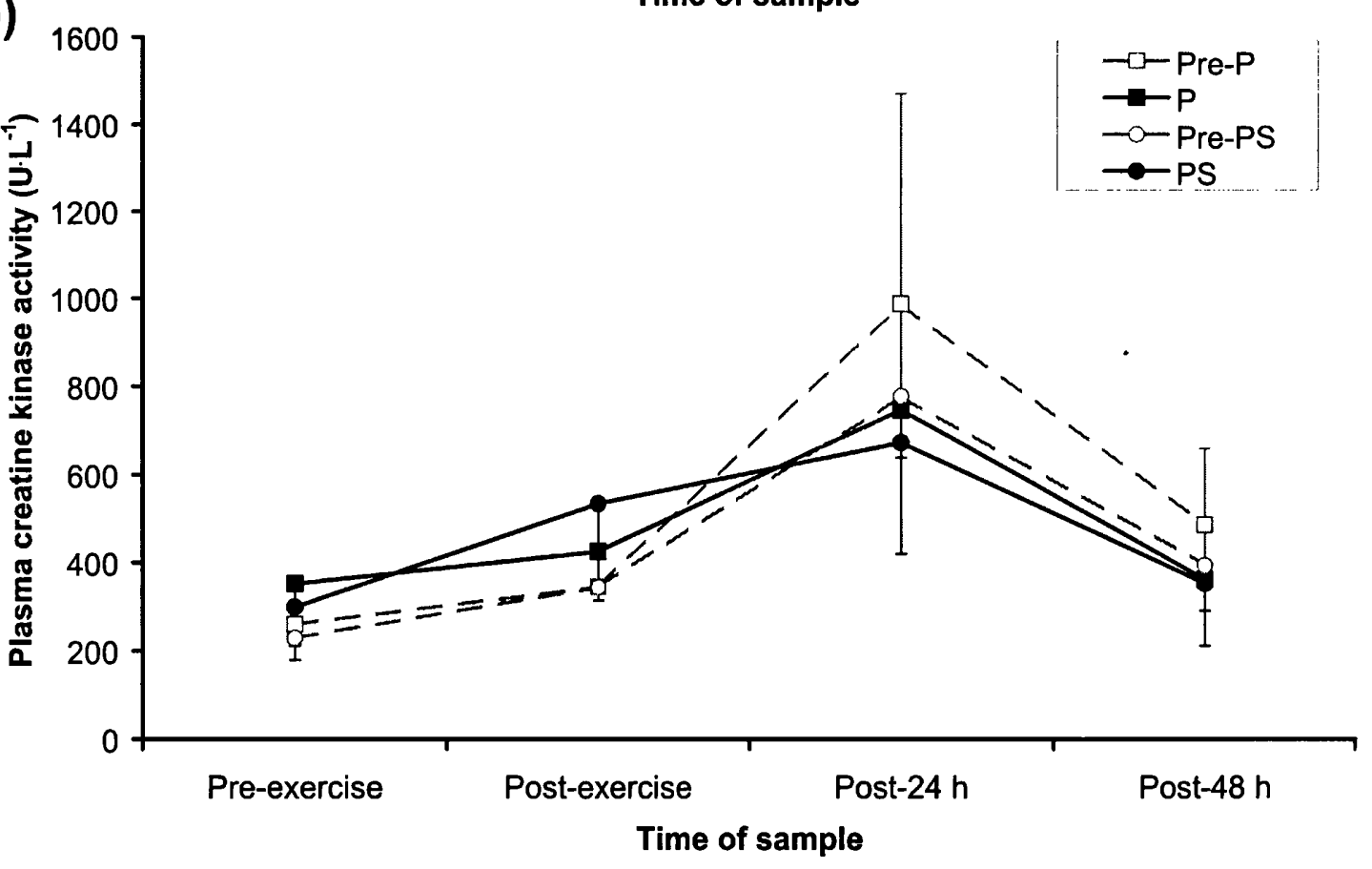

Figure 4.3 (a) Plasma myoglobin concentrations and (b) plasma creatine kinase activities throughout each trial. Values represent mean \pm SEM (N=8). Pre-P: trial prior to supplementation with placebo; P: trial supplemented with placebo; Pre-PS: trial prior to supplementation with phosphatidylserine; PS: trial supplemented with phosphatidylserine. 
Exercise caused increases in perceived soreness at Post-24 h in general soreness ratings (GenS) and in all of the reported lower body locations (QS, HS and GS); however, no differences were identified between trials (Table 4.2). Table 4.3 displays mean knee ROM and hip ROM at all time points by trials. Although these values were significantly reduced at Post- $24 \mathrm{~h}$, there were no differences between trials.

\section{Serum interleukin-6 Concentrations}

Serum IL-6 concentrations were elevated at Post-exercise by approximately $123 \pm$ $39 \%$ above Pre-exercise values (time of sample effect, $F=22.67 ; P<0.001$; partial $e t a^{2}=0.76$ ) in all trials (Figure 4.4). No differences were identified between the trials (trial effect, $P=0.871$ ).

Serum Hydroperoxide Concentrations

Serum HPO concentrations were significantly elevated at Post-exercise by

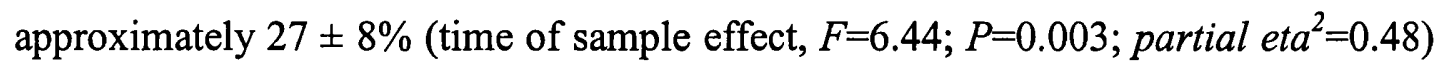
in all trials (Figure 4.5). There were no differences between trials (trial effect, $P=0.789$ ). 
Table 4.2 Perceived ratings of general soreness (GenS) quadriceps soreness (QS), hamstring soreness (HS), and gluteal soreness (GS) during all trials.

\begin{tabular}{|c|c|c|c|c|c|c|c|c|}
\hline & & $\begin{array}{c}\text { Pre-supp } \\
\text { Pre-exercise }\end{array}$ & $\begin{array}{l}\text { Pre-supp } \\
\text { Post-24 h }\end{array}$ & $\begin{array}{l}\text { Pre-supp } \\
\text { Post-48 h }\end{array}$ & $\begin{array}{c}\text { Post-supp } \\
\text { Pre-exercise }\end{array}$ & $\begin{array}{l}\text { Post-supp } \\
\text { Post-24 h }\end{array}$ & $\begin{array}{l}\text { Post-supp } \\
\text { Post-48 h }\end{array}$ & $\begin{array}{c}\text { Effect: } P \\
\text { trial, } \\
\text { timing }\end{array}$ \\
\hline GenS & $\mathrm{P}$ & $0.4 \pm 0.2$ & $2.4 \pm 0.6$ & $2.4 \pm 0.7$ & $0.4 \pm 0.2$ & $3.1 \pm 1.1$ & $1.5 \pm 0.6$ & 0.766 \\
\hline$(0-10)$ & PS & $0.5 \pm 0.6$ & $3.1 \pm 1.1$ & $2.6 \pm 0.7$ & $0.5 \pm 0.6$ & $2.3 \pm 0.6$ & $1.1 \pm 0.8$ & 0.002 \\
\hline QS & $P$ & $0.3 \pm 0.2$ & $1.5 \pm 0.6$ & $0.9 \pm 0.3$ & $0.3 \pm 0.2$ & $0.9 \pm 0.6$ & $0.3 \pm 0.3$ & 0.092 \\
\hline$(0-10)$ & PS & $0.3 \pm 0.2$ & $1.4 \pm 0.7$ & $1.1 \pm 0.4$ & $0.3 \pm 0.2$ & $1.4 \pm 0.7$ & $1.1 \pm 0.4$ & 0.043 \\
\hline HS & $P$ & $0.3 \pm 0.2$ & $1.1 \pm 0.4$ & $1.5 \pm 0.8$ & $0.3 \pm 0.2$ & $2.4 \pm 1.1$ & $0.8 \pm 0.4$ & 0.819 \\
\hline$(0-10)$ & PS & $0.4 \pm 0.2$ & $0.8 \pm 0.5$ & $0.9 \pm 0.5$ & $0.3 \pm 0.2$ & $1.3 \pm 0.5$ & $0.4 \pm 0.4$ & 0.024 \\
\hline GS & $P$ & $0.3 \pm 0.2$ & $2.9 \pm 0.8$ & $2.8 \pm 0.9$ & $0.4 \pm 0.2$ & $3.1 \pm 1.0$ & $0.9 \pm 0.4$ & 0.781 \\
\hline$(0-10)$ & PS & $0.4 \pm 0.2$ & $3.3 \pm 1.1$ & $3.1 \pm 1.2$ & $0.4 \pm 0.2$ & $2.3 \pm 0.5$ & $1.3 \pm 0.8$ & 0.001 \\
\hline
\end{tabular}

Values represent mean $\pm \mathrm{SEM}(\mathrm{N}=8)$. Pre-supp: pre-supplementation trials (trial 1 and trial 3); Postsupp: post-supplementation trial (trial 2 and trial 4); P: trial supplemented with placebo; PS: trial supplemented with phosphatidylserine. Effects present $P$-values for the main effect of trial and the main effect of time of sample. 
Table 4.3 Active range of motion during knee flexion (knee ROM) and hip flexion (hip ROM) during all trials.

\begin{tabular}{llccccccc}
\hline & & & & & & & & \\
& & Pre-supp & Pre-supp & Pre-supp & Post-supp & Post-supp & Post-supp & $\begin{array}{c}\text { Effect: } P \\
\text { trial, } \\
\text { timing }\end{array}$ \\
\hline Knee ROM & P & $118 \pm 2$ & $115 \pm 3$ & $113 \pm 3$ & $120 \pm 3$ & $113 \pm 2$ & $115 \pm 1$ & 0.783 \\
( $)^{\circ}$ & PS & $116 \pm 2$ & $113 \pm 2$ & $112 \pm 3$ & $117 \pm 2$ & $111 \pm 4$ & $116 \pm 3$ & 0.001 \\
Hip ROM & P & $84 \pm 5$ & $73 \pm 6$ & $78 \pm 7$ & $85 \pm 3$ & $84 \pm 2$ & $88 \pm 6$ & 0.877 \\
$\left(^{\circ}\right)$ & PS & $86 \pm 4$ & $84 \pm 2$ & $88 \pm 6$ & $86 \pm 4$ & $77 \pm 4$ & $76 \pm 2$ & 0.011 \\
\hline
\end{tabular}

Values represent mean \pm SEM (N=8). Pre-supp: pre-supplementation trials (trial 1 and trial 3); Postsupp: post-supplementation trial (trial 2 and trial 4); P: trial supplemented with placebo; PS: trial supplemented with phosphatidylserine. Effects present $P$-values for the main effect of trial and the main effect of time of sample. 


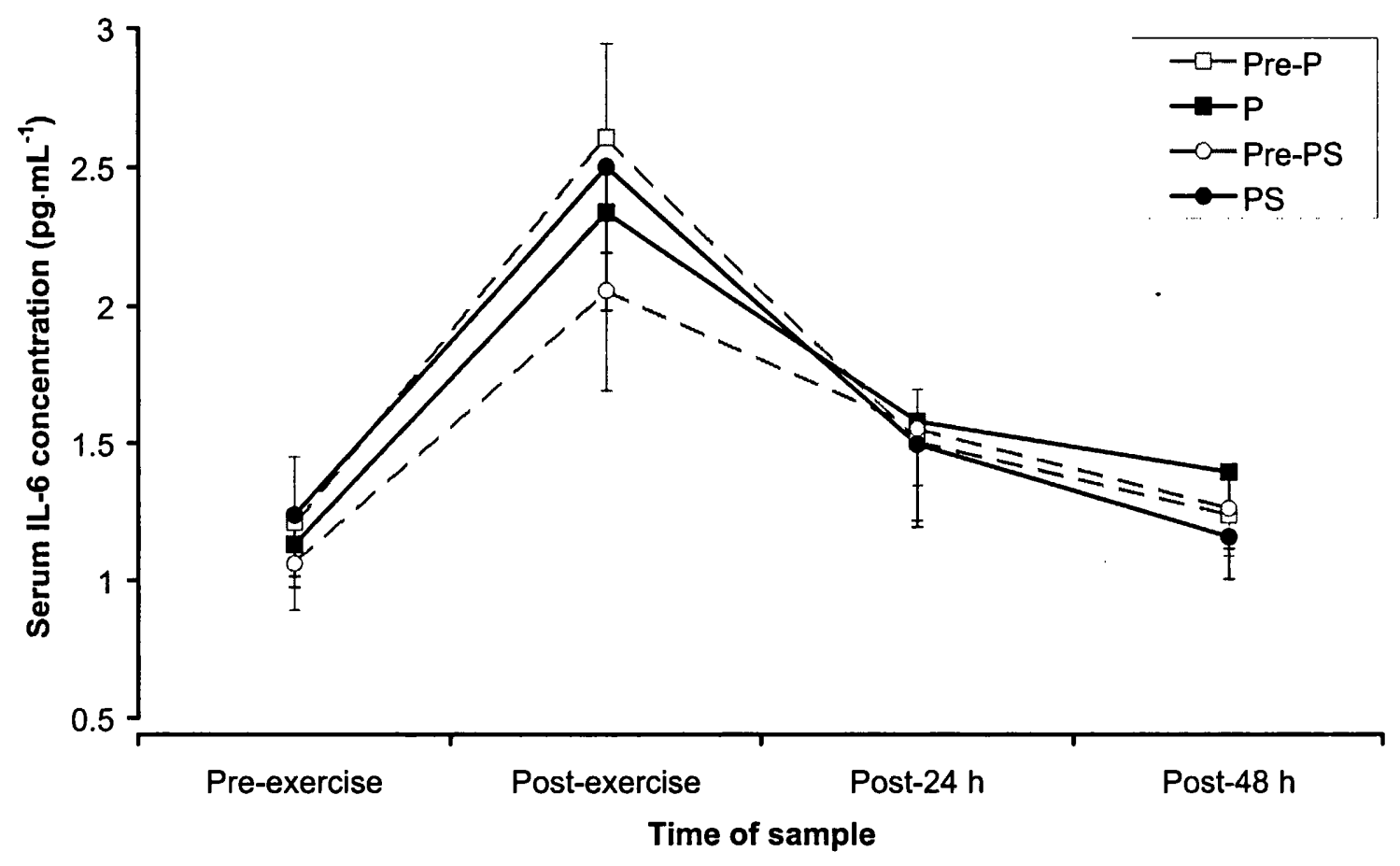

Figure 4.4 Serum interleukin (IL-6) concentrations throughout each trial. Values represent mean \pm SEM $(\mathrm{N}=8)$. Pre-P: trial prior to supplementation with placebo; $\mathrm{P}$ : trial supplemented with placebo; Pre-PS: trial prior to supplementation with phosphatidylserine; PS: trial supplemented with phosphatidylserine. 


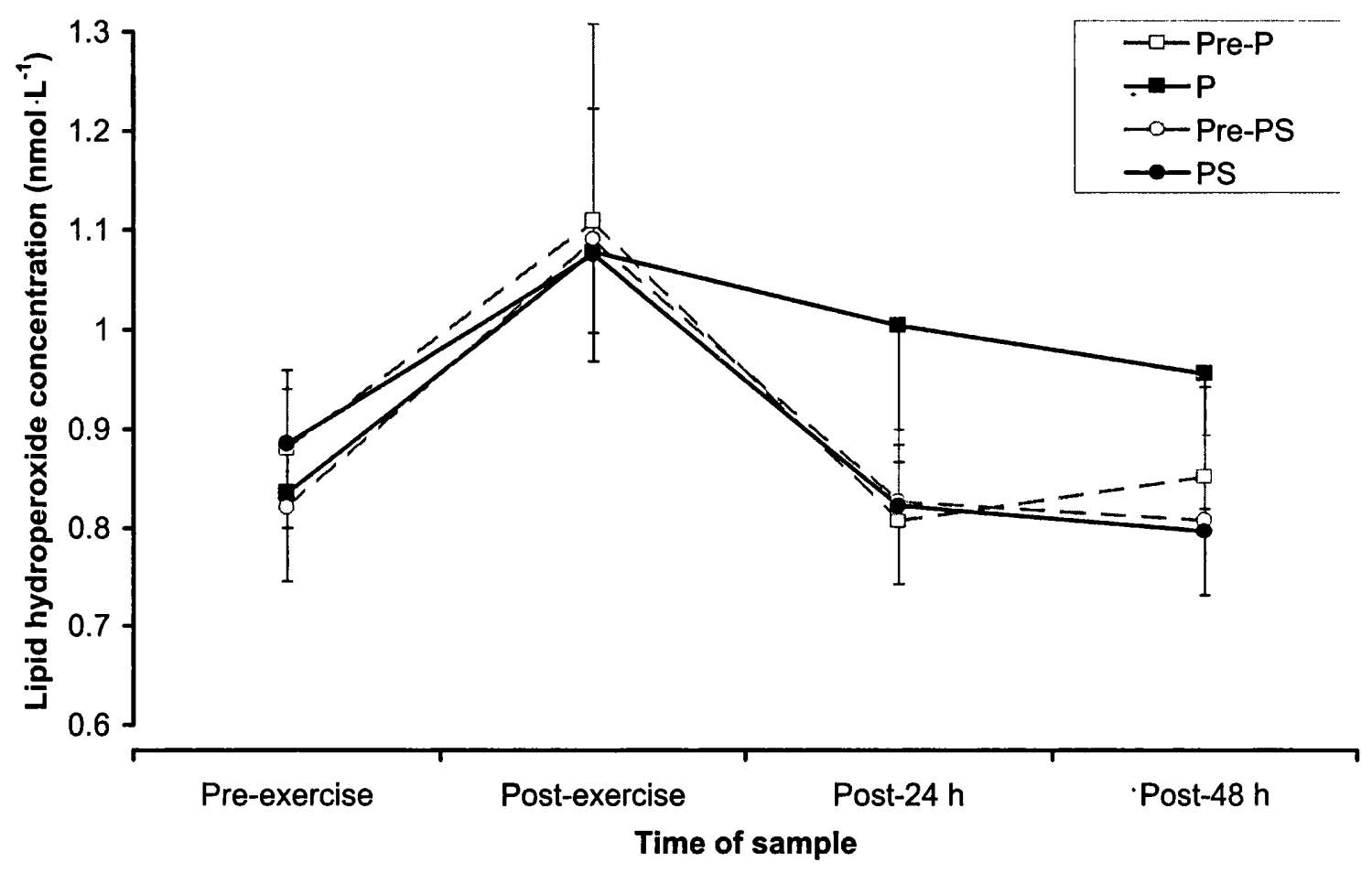

Figure 4.5 Serum hydroperoxide concentrations throughout each trial. Values represent mean \pm SEM $(\mathrm{N}=8)$. Pre-P: trial prior to supplementation with placebo; $\mathrm{P}$ : trial supplemented with placebo; Pre-PS: trial prior to supplementation with phosphatidylserine; PS: trial supplemented with phosphatidylserine. 
Average changes in conjugated diene concentrations at Pre-exercise, Post-exercise, Post-24 $\mathrm{h}$ and Post-48 $\mathrm{h}$ were $14.2 \pm 0.6,15.6 \pm 0.8,14.8 \pm 1.0,14.2 \pm 0.8 \mu \mathrm{mol} \cdot \mathrm{L}^{-1}$, respectively (time of sample effect, $P=0.077$; trial effect, $P=0.338$ ) (Figure 4.6). Post-exercise $t_{1 / 2 \max }$ LDL oxidation were elevated from Pre-exercise (time of sample effect, $P=0.001$ ), with average Pre-exercise, Post-exercise, Post-24 h and Post- $48 \mathrm{~h}$ values being $79.2 \pm 2.2,87.2 \pm 2.5,86.1 \pm 2.6,82.5 \pm 2.7 \mathrm{~min}$, respectively (Figure 4.7). No differences existed between trials (trial effect, $P=0.192$ ).

\section{Plasma Antioxidant Vitamin Concentrations}

Plasma concentrations of vitamin $\mathrm{C}, \alpha$-tocopherol, $\alpha$-carotene, $\beta$-carotene, lycopene were similar throughout the duration of each trial and were not significantly different between trials (Table 4.4). Plasma $\gamma$-tocopherol concentrations were significantly higher in PS when compared with all other trials (trial effect, $F=5.32$; $P=0.008$; partial eta ${ }^{2}=0.47$ ) (Table 4.4). 


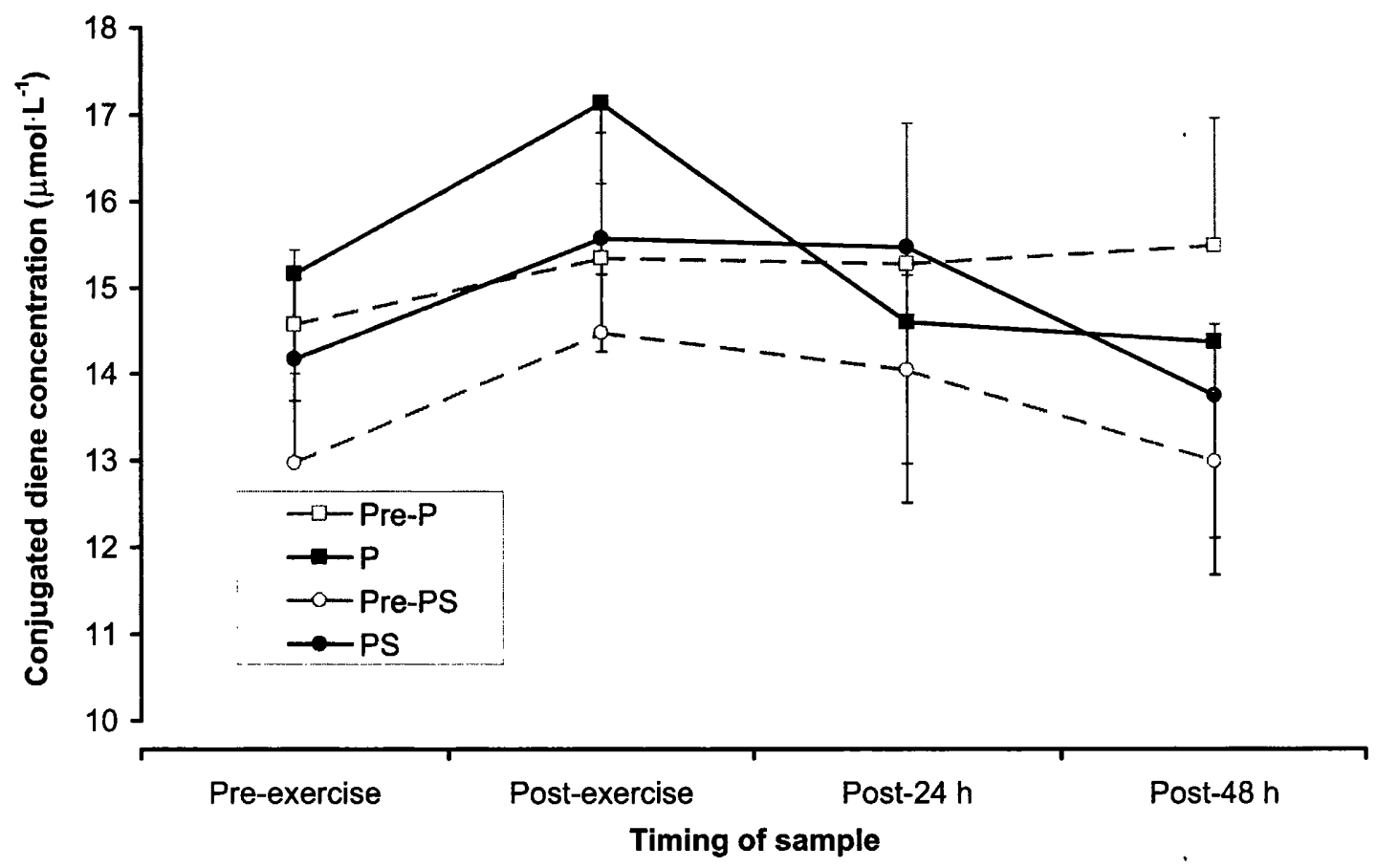

Figure 4.6 Change in conjugated diene concentrations throughout each trial. Values represent mean \pm SEM $(\mathrm{N}=8)$. Pre-P: trial prior to supplementation with placebo; $\mathrm{P}$ : trial supplemented with placebo; Pre-PS: trial prior to supplementation with phosphatidylserine; PS: trial supplemented with phosphatidylserine. 


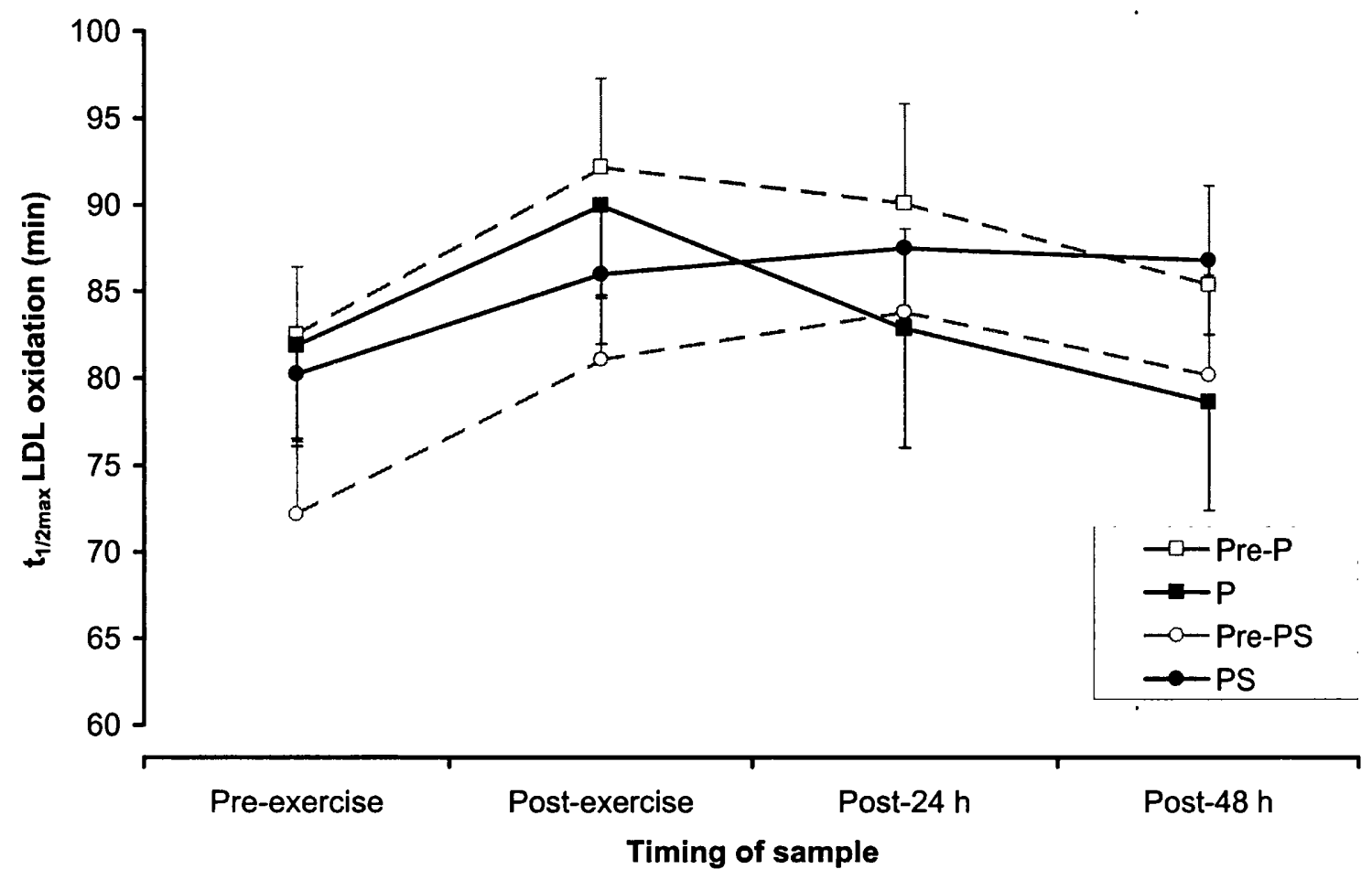

Figure 4.7 Time to half of maximum low density lipoprotein oxidation ( $t_{1 / 2 \max } \mathrm{LDL}$ oxidation) throughout each trial. Values represent mean \pm SEM (N=8). Pre-P: trial prior to supplementation with placebo; P: trial supplemented with placebo; Pre-PS: trial prior to supplementation with phosphatidylserine; PS: trial supplemented with phosphatidylserine. 


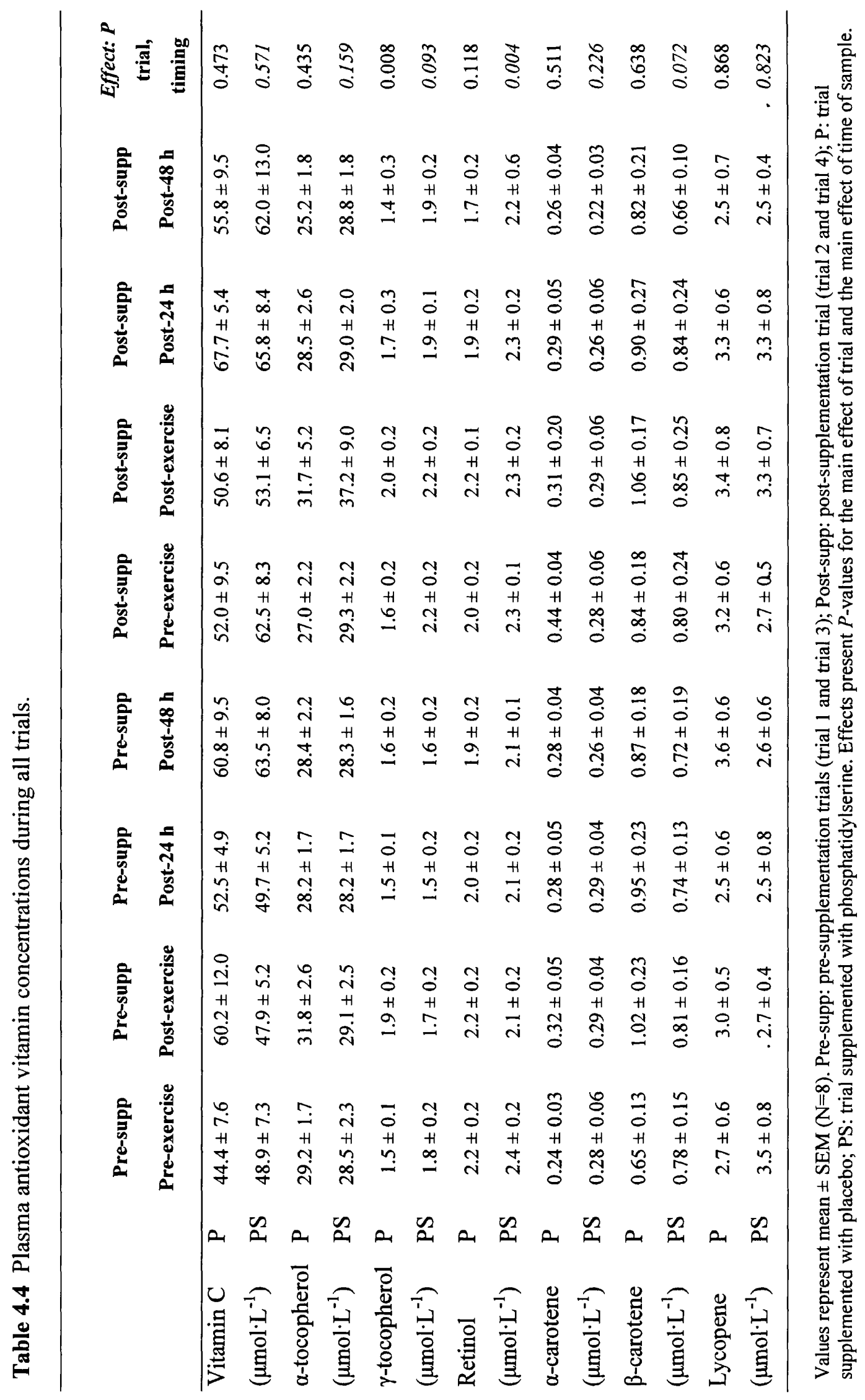


Feeling States

No significant temporal changes were observed in revitalisation (time of sample effect, $P=0.295$ ), positive engagement (time of sample effect, $P=0.357$ ), tranquillity (time of sample effect, $P=0.671$ ) or physical exhaustion (time of sample effect, $P=0.081$ ) (Table 4.5). Additionally, no differences were identified between trials in any of the EFI subscales (trial effect, $P \geq 0.121$ ) (Table 4.5). 


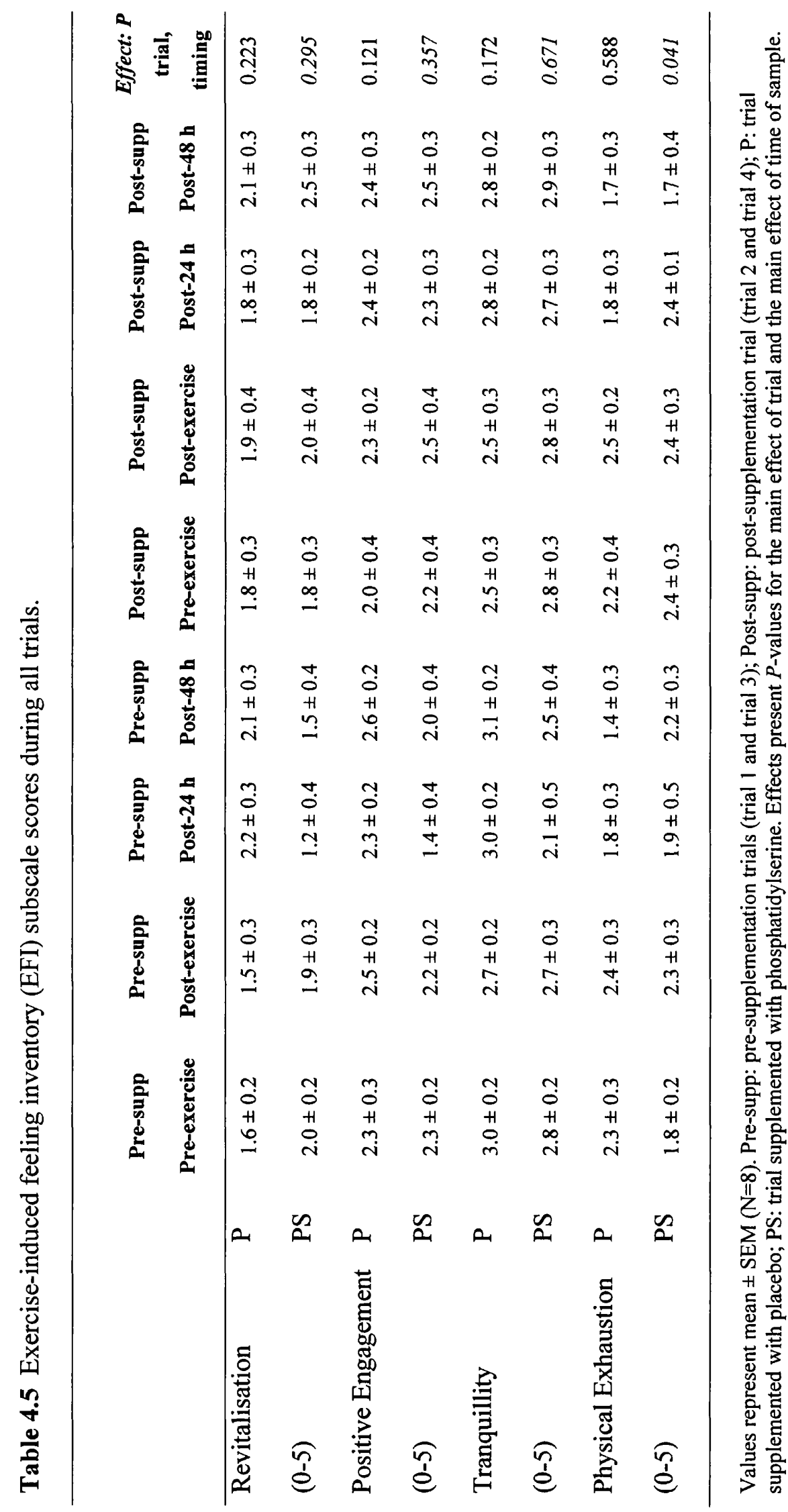




\subsection{DISCUSSION}

The main findings of this study were that the supplementation regime $\left(750 \mathrm{mg} \cdot \mathrm{d}^{-1} \mathrm{~S}\right.$ PtdSer administered for $7 \mathrm{~d}$ prior to and continued for $2 \mathrm{~d}$ following exercise) was not effective in attenuating markers of muscle damage, inflammation and oxidative stress that followed prolonged downhill treadmill running in previously familiarised individuals.

In light of the finding that supplementation with $750 \mathrm{mg} \cdot \mathrm{d}^{-1} \mathrm{~S}-\mathrm{PtdSer}$ for $10 \mathrm{~d}$ tended to improve performance during exhaustive intermittent running (study 1 ), the current exercise protocol was chosen in order to provide each subject with a repeatable dose of exercise that was intended to cause muscle damage, oxidative stress, acute inflammation and DOMS. Indeed, this exercise protocol led to increases in markers of muscle damage (plasma $\mathrm{Mb}$ concentrations and $\mathrm{CK}$ activities), lipid peroxidation (serum HPO concentrations), inflammation (serum IL-6 concentrations) and DOMS (perceived soreness).

The 3-way interactions for the analysis of trial 1 and 3 (pre-supplementation control trials) were all non-significant. These data confirmed that the subject groups reacted similarly during these trials. Although the washout kinetics of PtdSer are currently unknown, there is no evidence to suggest that an effect of supplementation lasted longer than the washout period provided. 
As anticipated, plasma cortisol concentrations were positively correlated with plasma ACTH concentrations. Plasma concentrations of cortisol and ACTH decreased after eccentric exercise. This finding probably reflects the low metabolic demand that is associated with downhill running at the current relative intensity and is concurrent with previous data (Smith et al., 1989). Alterations in the concentrations of these hormones within the circulation have been interpreted to reflect changes in the hypothalamo-pituitary-adrenal (HPA) axis (Monteleone et al., 1990). Consequently, it is likely that this bout of exercise did not cause a stress induced increase in hypothalamic corticotrophin-releasing factor that has concurrent stimulatory effects on a range of regulatory hormones from the anterior pituitary (including growth hormone) and sympathoadrenal system (such as adrenaline and noradrenaline). Therefore, it is unlikely that the intensity of exercise was sufficient to augment the mobilisation of neutrophils into the peripheral circulation as mediated by exercise induced increases in catecholamines, cortisol and growth hormone (Suzuki et al., 1999). Interestingly, S-PtdSer supplementation did not alter the pattern of ACTH and cortisol release. Fahey and Pearl (1998) reported that similar doses of S-PtdSer reduced serum cortisol concentrations following resistance training, leading these investigators to support the efficacy of PtdSer to modulate the neuroendocrine response to overtraining. The disparity in these findings might be partly explained by the different mode of exercise used in these respective studies; downhill running, unlike resistance training, did not stimulate the stress-induced activation of the HPA axis.

The elevations in plasma $\mathrm{Mb}$ concentrations and $\mathrm{CK}$ activities were comparable in magnitude and followed similar temporal patterns to those that have been previously 
reported following downhill running protocols (Peake et al., 2005). This protocol also led to increased soreness (GenS, QS, HS and GS) and decreased the ranges of motion recorded during active knee and hip flexion on the day following exercise. Furthermore, the time course of the changes in the ratings of soreness, joint flexibility, plasma $\mathrm{Mb}$ concentrations and plasma $\mathrm{CK}$ activities following eccentric exercise were similar across all trials; consequently, it was concluded that supplementation with S-PtdSer did not affect muscle damage and other important corollaries induced by an acute bout of eccentric exercise. In contrast, a similar SPtdSer supplementation regime has been demonstrated to reduce muscle soreness during intense weight training (Fahey and Pearl, 1998). These authors postulated that exogenous PtdSer might have improved cell membrane stability and/or reduced protein degradation by attenuating the stress-induced activation of the HPA axis. Although markers of protein catabolism were not measured in the present study, there was no evidence to suggest that S-PtdSer affected protein degradation following eccentric exercise.

Downhill running led to significant increases in Post-exercise serum IL-6 concentrations that were comparable to those previously reported to follow similar exercise protocols (Peake et al., 2005). The magnitude of the IL-6 increases following downhill running were relatively small when compared with the changes that have been reported to occur following prolonged endurance exercise (Suzuki et al., 2000). This finding probably reflects both the type and duration of activity undertaken. Although the stimulus for eccentric exercise induced IL-6 remains largely unknown, it is likely that mechanical damage to muscle tissue led to the rapid invasion of neutrophils (Tidball, 2005) which produce free radicals that 
stimulate cytokine production. However, supplementation did not affect circulatory concentrations of IL-6 in the current study.

Serum HPO concentrations were elevated to a similar extent following exercise in all trials; consequently, exercise caused an increase in free radical production that overwhelmed the antioxidant defences and, independent of supplementation, contributed to comparable amounts of lipid peroxidation.

Exercise did not significantly affect the susceptibility of LDL to copper-induced oxidation as measured by changes in conjugated diene concentrations. However, $t_{1 / 2 \max }$ LDL oxidation times were elevated at post-exercise. As $t_{1 / 2 \max }$ LDL oxidation is a measure of resistance to copper-induced oxidation, the increase following exercise might reflect an increase in the mobilization of antioxidant defences following downhill running. Supplementation did not influence susceptibility or resistance of LDL to in-vitro peroxidation.

Supplementation led to significant increases in plasma $\gamma$-tocopherol concentrations throughout the PS trial. This increase is in agreement with the findings from experimental study 1 ; however, the mechanism for increasing plasma $\gamma$-tocopherol following supplementation are unclear. Nevertheless, there is insufficient evidence to suggest that the elevations in plasma $\gamma$-tocopherol concentrations and probable increases in tissue $\gamma$-tocopherol affected oxidative stress or inflammation caused by eccentric exercise. In addition, supplementation had no effect on concentrations of 
the other plasma antioxidant vitamins (vitamin $\mathrm{C}, \alpha$-tocopherol, $\gamma$-tocopherol, retinol, $\alpha$-carotene, $\beta$-carotene and lycopene).

Downhill running did not affect any of the EFI subscales. The EFI was developed to assess distinct feeling states that occur during exercise (Gauvin and Rejeski, 1993) and these scales have been demonstrated to be sensitive to change during exercise in recreationally active males. This difference probably reflects the low metabolic demand associated with this downhill running protocol and suggests that DOMS does not change the feeling states that are measured by these subscales. The lack of change in these feeling states might have contributed to the lack of an identifiable difference between trials; nevertheless, the current data does not suggest that S-PtdSer modifies feeling states before or following downhill running.

In summary, short-term S-PtdSer supplementation before and following exercise did not attenuate perceived soreness and markers of muscle damage, inflammation and oxidative stress caused by moderate intensity downhill running. These findings indicate that the current supplementation regime is not effective in reducing the deleterious effects of eccentric muscle activity. 


\section{CHAPTER FIVE}

\section{EXPERIMENTAL STUDY THREE}

Effects of phosphatidylserine supplementation on

exercise capacity during staged intermittent cycling 


\subsection{INTRODUCTION}

Phosphatidylserine (PtdSer) is a naturally occurring phospholipid nutrient that appears to be essential to the functioning of all mammalian cells (Blokland et al., 1999). In humans, PtdSer is most concentrated in organs with high metabolic activity, such as brain, heart, skeletal muscle and liver (Diagne et al., 1984). The biochemical actions of PtdSer include: [1] regulation of calcium ion uptake, [2] regulation of substrate binding (e.g., opiate and glutamate), and [3] the stimulation of specific enzymes activities (e.g., ATPase and acetylcholinesterase) (Pepeu et al., 1996). The discovery of these actions has led investigators to examine the pharmacological effects of PtdSer supplementation in humans. Traditionally, PtdSer supplements were derived from bovine cortex (BC-PtdSer); however, this source is now considered unsuitable due to the risk of transferring infectious diseases and soyderived PtdSer (S-PtdSer) has emerged as a safe alternative. Although Blokland $e t$ al. (1999) found no differences between the bioactivity of S-PtdSer and BC-PtdSer in rats, it remains unclear whether the source of the supplement is an important factor in determining the pharmacological actions of PtdSer in humans.

The majority of PtdSer supplementation studies have evaluated higher brain functions; indeed, BC-PtdSer has been shown to improve a variety of cognitive functions that include memory and verbal response in aging patients (Amaducci and SMID group, 1988; Cenacchi et al., 1993), and more recently S-PtdSer has been demonstrated to enhance mood in a subsection of young individuals during mental stress (Benton et al., 2001). Few studies have investigated the effects of PtdSer supplementation prior to exercise (Monteleone et al., 1990; Monteleone et al., 1992; 
Fahey and Pearl, 1998) and the available data suffer from the methodological limitation of using cross-over supplementation designs while the washout kinetics of PtdSer remains unclear (Pepeu et al., 1996). Orally administered BC-PtdSer (800 $\mathrm{mg} \mathrm{d}^{-1}$ ) has been reported to attenuate the serum cortisol response to acute exercise stress (Monteleone et al., 1992). Additionally, Fahey and Pearl (1998) reported that oral supplementation with $800 \mathrm{mgd}^{-1}$ S-PtdSer was effective in reducing serum cortisol concentration following intensive resistance training. These findings suggest that both BC-PtdSer and S-PtdSer act to partly counteract the stress-induced activation of the hypothalamo-pituitary-adrenal (HPA) axis, although the significance of altering this neuroendocrine response on metabolic function (such as substrate utilisation) during exercise has not been evaluated.

Assuming that exogenous PtdSer is effectively incorporated into cardiac and skeletal muscles, the aforementioned biochemical actions of PtdSer have the potential to influence mitochondrial enzymatic activity. Therefore, PtdSer supplementation might affect 'metabolic inertia', a proposed determinant of the primary oxygen uptake $\left(\mathrm{V}_{2}\right)$ kinetic response to exercise (Grassi, 2000). Consequently, it was hypothesised that PtdSer supplementation could reduce accumulated oxygen deficit and improve exercise tolerance.

Therefore the purpose of the current study was to investigate the efficacy of shortterm S-PtdSer supplementation on exercise capacity, neuroendocrine function, oxygen uptake kinetic response, and perceived feeling states during and immediately following intermittent cycling. 
$\mathrm{HO}_{1}$ : Intermittent cycling will not influence markers of neuroendocrine function, oxygen uptake kinetic responses, and perceived feeling states.

$\mathrm{HO}_{2}$ : Supplementation with S-PtdSer will not influence exercise time to

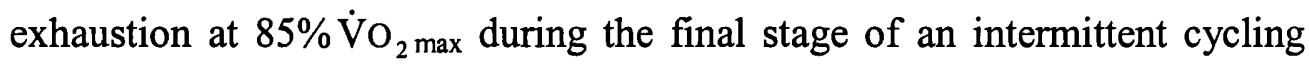
protocol.

$\mathrm{HO}_{3}$ : Supplementation with S-PtdSer will not influence markers of neuroendocrine function, and oxygen uptake kinetics during and following staged intermittent cycling.

$\mathrm{HO}_{4}$ : Supplementation with S-PtdSer will not alter perceived feeling states during and following staged intermittent cycling. 


\subsection{METHODS}

\section{Subjects}

Sixteen healthy males volunteered to participate in this study; fourteen subjects (Table 5.1) completed all the study requirements and were included in the analyses. All subjects were informed about the potential risks of the study and gave written informed consent for their participation in the study, which was approved by a university ethics committee. In addition, all subjects were informed that they might be asked to consume a supplement rich in S-PtdSer; however, there are currently no reported, or inferred, side effects associated with S-PtdSer supplementation (Pepeu et al., 1996; Jorissen et al., 2002). The experimental procedures were in accordance with the policy statement of the American College of Sports Medicine. No subject had prior history of cardiovascular or respiratory disease and all subjects were nonsmokers. Potential subjects attended an interview prior to undertaking the study and were subsequently excluded if they had taken nutritional supplements in the last 8 weeks.

\section{Experimental Design}

Before the main exercise trials, all subjects visited the laboratory on two occasions in order to complete an incremental exercise test and a familiarisation trial. Subjects then performed two main exercise trials, which consisted of staged intermittent cycling, separated by $16.0 \pm 1.3 \mathrm{~d}$. Approximately $5 \mathrm{~d}$ after completion of the first main exercise trial the subjects were assigned, in a randomised double-blind fashion, 
Table 5.1 Subject characteristics for phosphatidylserine supplementation group (PS) and Placebo group (P).

\begin{tabular}{lccc}
\hline Characteristic & PS (N=7) & P (N=7) & P-value \\
\hline Age (dec. years) & $23.4 \pm 1.9$ & $22.2 \pm 1.1$ & 0.638 \\
Mass (kg) & $84.9 \pm 3.8$ & $87.7 \pm 3.2$ & 0.630 \\
Height (m) & $1.79 \pm 0.01$ & $1.81 \pm 0.02$ & 0.528 \\
Maximal oxygen uptake $\left(\mathrm{ml} \cdot \mathrm{kg}^{-1} \cdot \mathrm{min}^{-1}\right)$ & $43.8 \pm 2.0$ & $42.4 \pm 1.7$ & 0.645 \\
\hline
\end{tabular}

Values are mean \pm SEM. $P$-value calculated using independent sample $t$-test. 
to either a PS group or a placebo $(\mathrm{P})$ group and instructed to take supplements for the 10 days prior to trial two. The experimental design is illustrated in Figure 5.1.

The PS group ingested $750 \mathrm{mg} \cdot \mathrm{d}^{-1} \mathrm{~S}-\mathrm{PtdSer}$ and the $\mathrm{P}$ group ingested a weight matched glucose polymer. The S-PtdSer supplements were manufactured using the method of specific transesterification of soybean lecithin and then blended with additional soybean lecithin to provide a concentration of $20 \%$ PtdSer (Lucas Meyer; Hamburg, Germany). Both supplements were administered in capsules and placed in generic packaging. Subjects were instructed to maintain their normal diet and activity patterns throughout the study. Food was weighed and recorded by the subjects for two days prior to each exercise trial and for the day afterwards. Analysis using commercial software (CompEat v5.8.0; Nutrition Systems, UK) revealed that there were no differences in energy intake or dietary composition between supplementation groups or trials. The daily diet comprised of $11.2 \pm 0.6 \mathrm{MJ} \cdot \mathrm{d}^{-1}$, of which $52 \pm 3 \%, 30 \pm 1 \%, 16 \pm 1 \%$ and $2 \pm 1 \%$ of energy intake was obtained from carbohydrates, fat, protein and alcohol, respectively. In addition, the subjects were instructed to abstain from strenuous exercise for three days prior to and two days following each trial. At the completion of the study all subjects gave their verbal assurance that they had complied with all instructions. 

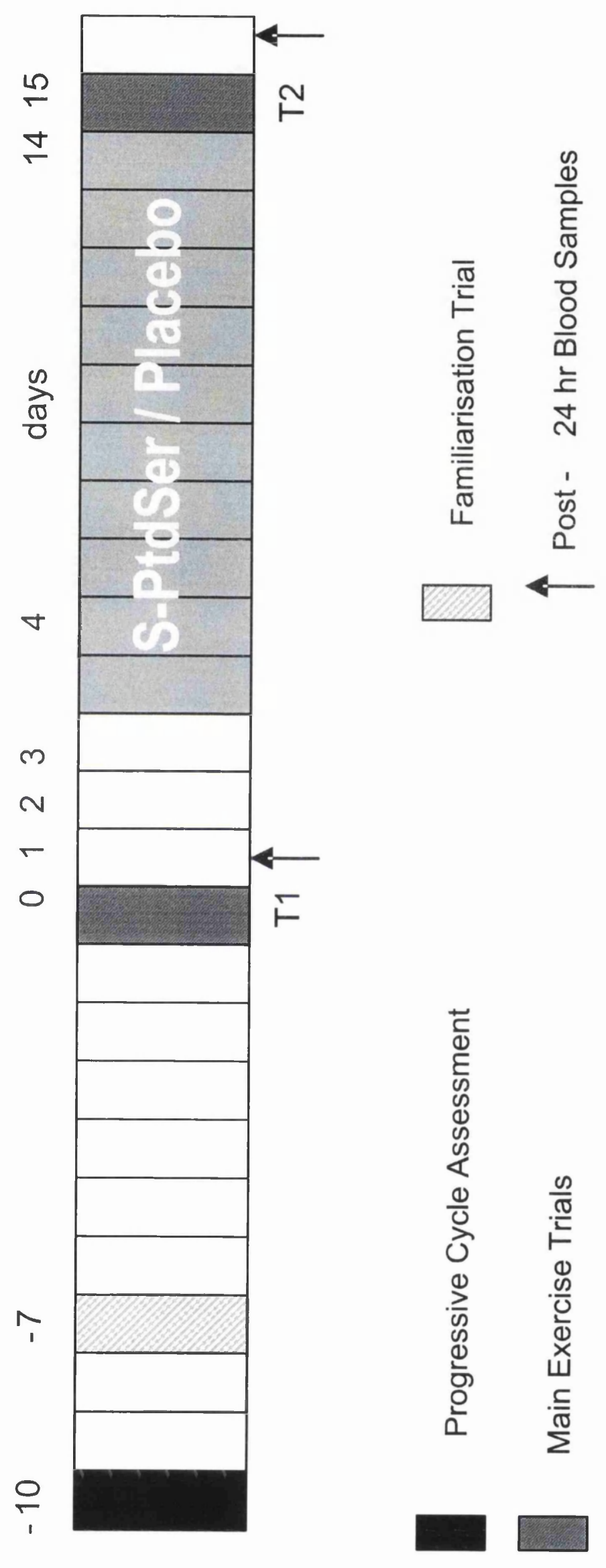

हี 


\section{Procedures}

During their initial visit to the laboratory the subjects completed an incremental exercise test on an electromagnetically braked cycle ergometer (Lode Excalibur Sport; Lode, Holland). The work rate began at $60 \mathrm{~W}$ and thereafter increased in 30W increments every 3 min until volitional exhaustion. Subjects were instructed to pedal at a constant cadence between 75 and $85 \mathrm{rev} \cdot \mathrm{min}^{-1}$. Heart rate (Polar S810; Polar Electro Oy, Finland) and breath-by-breath respiratory parameters (Oxycon Pro; Jaeger, Germany) were simultaneously recorded. The test was used to identify maximal oxygen uptake $\left(\dot{\mathrm{V}}_{2}\right.$ max $)$ and also to calculate, using linear regression, the work rates required for the staged intermittent exercise protocol.

The subjects then completed a staged intermittent exercise protocol on three separate occasions. The intermittent protocol required subjects to complete three 10-min stages of cycling at work rates calculated to elicit 45,55 and $65 \% \dot{\mathrm{V}}_{2}$ max , followed by a final exercise bout at $85 \% \dot{\mathrm{VO}}_{2}$ max that was continued until exhaustion (an inability to maintain a cadence of $60 \mathrm{rev} \cdot \mathrm{min}^{-1}$ despite verbal encouragement). All exercise stages were interspaced with 5-min passive rest periods. The first exercise trial served to familiarise the subjects with the intermittent exercise protocol, and the subjects remained blind to their exercise times to exhaustion throughout the study in order to prevent an anticipated trial-order effect. The subsequent main exercise trials (T1 and T2) were completed 14-21 d apart. Breath-by-breath respiratory data (Oxycon Pro; Jaeger, Germany) and heart rate (Polar S810; Polar Electro Oy, Finland) were monitored throughout T1 and T2. Exercise-induced feeling inventory (EFI) (Gauvin and Rejeski, 1993) was completed at Pre-exercise and following each 
exercise stage. Blood samples were taken by venepuncture (Vacutainer system; Becton-Dickinson Ltd, UK) from an antecubital vein at Pre-exercise, on completion of the $55 \% \dot{\mathrm{VO}}_{2} \max \left(\right.$ Post-55\%) and $65 \% \dot{\mathrm{V}}_{2} \max$ (Post-65\%) exercise stages, 20 min after the completion of the trial (Post-exercise) and 24 hours after the trial (Post$24 \mathrm{~h})$. An additional capillary blood sample was taken immediately after exhaustion (Post-85\%) for the analysis of blood lactate and glucose concentrations.

\section{Analysis of Oxygen Uptake and Heart Rate}

Breath-by-breath oxygen uptake, carbon dioxide production and 5-s heart rate data were averaged for the last minute of exercise for all exercise intensities. Subsequently non-protein respiratory exchange ratios were used to calculate the rates of carbohydrate and fat oxidation for Pre-exercise and during each exercise stage up to and include $65 \% \mathrm{~V}_{2}$ max as previously described (Van Loon et al., 2001), using equations derived by Peronnet and Massicotte (1991). Linear regression was applied to the final 3 min of oxygen uptake data for each of these stages and the $95 \%$ confidence interval for the slope was inspected; steady state was assumed if the $95 \%$ confidence interval included zero.

In addition, breath-by-breath oxygen uptake data were initially edited to remove occasional errant breaths (from coughs, sighs or swallows) when values were greater than four standard deviations from the local mean (Rossiter et al., 2000). The data for all exercise stages and rest periods were interpolated, using a cubic spline, in 1-s 
intervals. The data for moderate intensity exercise $\left(45-65 \% \dot{\mathrm{V}}_{2}\right.$ max $)$ and subsequent rest periods during each trial were normalised (normalised to the respective last minute $\dot{\mathrm{VO}}_{2}$ values), time-aligned and averaged. The data for the final bout $(85 \%$ $\dot{\mathrm{V}}_{2}$ max) were modelled separately, as the oxygen uptake response to different intensity domains (below and above lactate threshold) have been suggested to differ (Ozyener et al., 2001). The traditionally defined phase II oxygen uptake on-kinetic response (Whipp et al., 1982) was determined for $45-65 \% \dot{\mathrm{V}}_{2} \max$ and $85 \% \dot{\mathrm{V}}_{2}$ max. The end of phase I was set to $20 \mathrm{~s}$ after the onset of constant-load exercise and a mono-exponential function (Equation 1) was fitted between the end of phase I and 3 min of exercise (Schneider et al., 2002) using iterative non-linear regression techniques (SPSS version 12.0; SPSS Inc., IL). Similarly, the off-kinetic response to each exercise stage was determined using a mono-exponential function (Equation 2) after omission of the first $20 \mathrm{~s}$ of post-exercise data (Figure 5.2)

(Equation 1)

$$
\dot{\mathrm{V}} \mathrm{O}_{2}(t)=\dot{\mathrm{V}} \mathrm{O}_{2}(B)+G \cdot\left(1-e^{-(\mathrm{t}-\mathrm{TD}) / \tau}\right)
$$

(Equation 2)

$$
\dot{\mathrm{V}} \mathrm{O}_{2}(t)=\dot{\mathrm{V}} \mathrm{O}_{2}(E E)-G^{\prime} \cdot\left(1-e^{-\left(\mathrm{t}-\mathrm{TD} \mathrm{D}^{\prime}\right) / \tau^{\prime}}\right)
$$

where $\dot{\mathrm{V}} \mathrm{O}_{2}(t)$ is oxygen uptake at time $t, \dot{\mathrm{V}}_{2}(B)$ is baseline oxygen uptake (average $\dot{\mathrm{V}}_{2}$ during the minute prior to the onset of exercise), $\dot{\mathrm{VO}}_{2}(E E)$ is end of exercise oxygen uptake (average $\dot{\mathrm{VO}}_{2}$ during the last minute of exercise), $G$ is the primary gain (i.e., the calculated change in oxygen uptake), TD is the on-kinetic time delay and $\tau$ is the time constant (prime mark designates off-kinetics parameters). Mean response times for the on-kinetic responses $\left(\mathrm{MRT}_{\text {on }}\right)$ and offkinetic responses ( $\mathrm{MRT}_{\text {off }}$ ) were calculated as $\mathrm{TD}+\tau$ and $\mathrm{TD}^{\prime}+\tau^{\prime}$, respectively. 


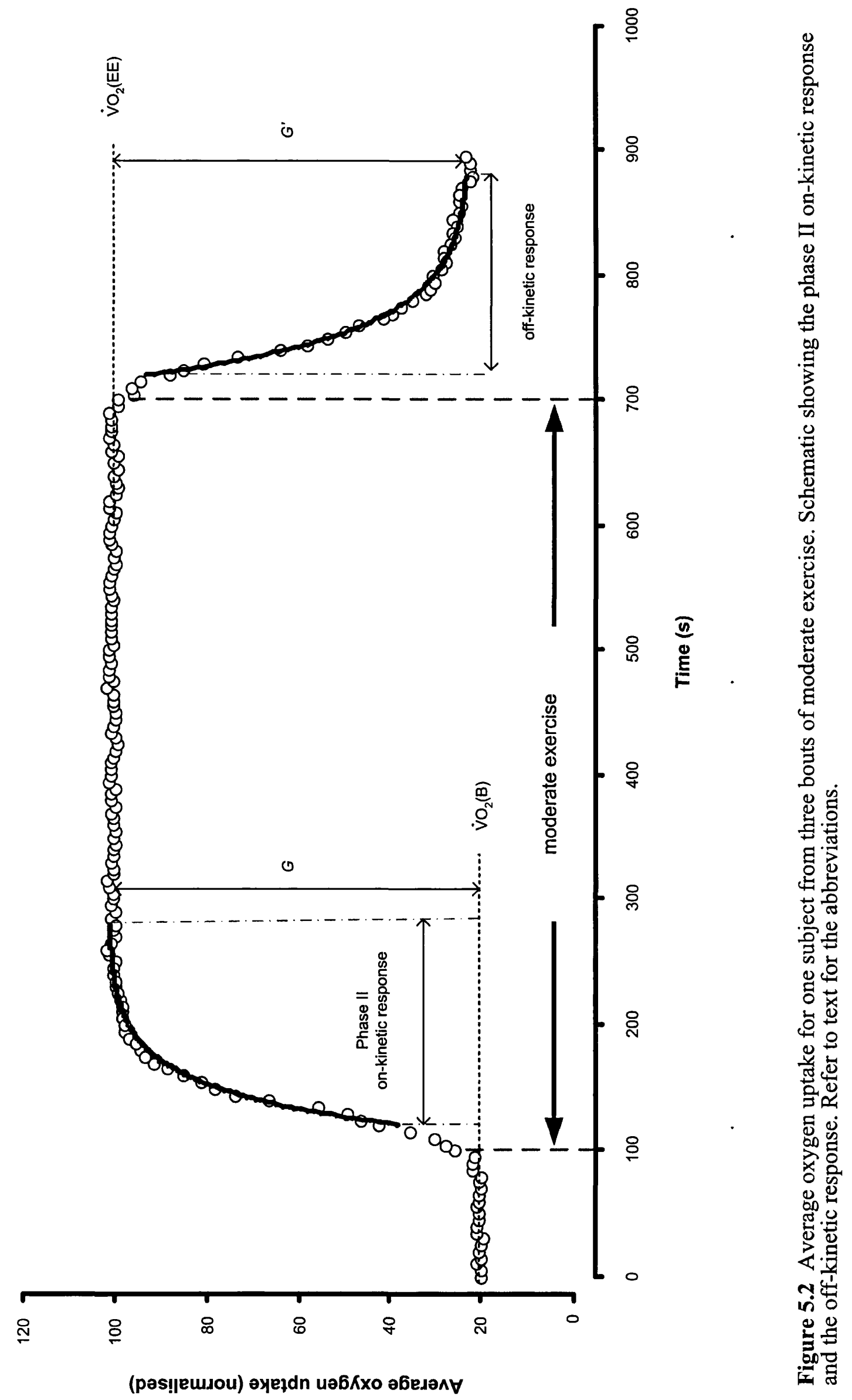




\section{Blood Sampling and Analysis}

Venous blood was collected in a 5-mL container (Becton-Dickinson Ltd, UK) containing the anticoagulant ethylenediamine tetra-acetic acid (EDTA). Several small aliquots were removed for the triplicate determination of blood lactate and glucose concentration (YSI 2300 Stat, Yellow Springs Instruments, USA), blood haemoglobin concentration (Hemocue Ltd, UK), haematocrit (Micro haematocrit MK IV, Hawksley, England) and changes in plasma volume as previously described (Dill and Costill, 1974). The remaining blood was centrifuged at $3000 \mathrm{x}$ g for 15 min to obtain plasma, which was subsequently dispensed and frozen at $-70^{\circ} \mathrm{C}$. Two additional 7-mL blood sample were collected in serum separation tubes (BectonDickinson Ltd, UK), left to stand for $15 \mathrm{~min}$, then centrifuged at $3000 \mathrm{x} \mathrm{g}$ for $15 \mathrm{~min}$ to obtain serum. The serum was transferred to appropriate containers and subsequently frozen at $-70^{\circ} \mathrm{C}$. Serum cortisol concentrations were determined using an automated time-resolved fluoroimmunoassay (AutoDELFIA ${ }^{\mathrm{TM}}$ Cortisol kit, Perkin Elmer, Life Sciences, UK).

\section{Perceived Feeling States}

Subjects were instructed to respond to the EFI (Appendix F) as described in detail by Gauvin and Rejeski (1993). Briefly, the subjects rated their feelings using the 12item adjective scale on an analogue scale from 0 (do not feel) to 5 (feel very strongly). The appropriate adjectives were averaged to obtain four perceived feeling states (positive engagement, revitalisation, tranquillity and physical exhaustion) at 
each time point as previously described (Gauvin and Rejeski, 1993). The EFI was specifically developed to assess distinct feeling states that occur during stages of exercise and psychometric studies have indicated concurrent and discriminant validity (Gauvin and Rejeski, 1993). Moreover, the EFI has been demonstrated to be sensitive to interventions involving recreational active individuals (Lacaille et al., 2004).

\section{Statistical Analysis}

Statistical analysis was carried out using SPSS software (version 12.0; SPSS Inc., IL). Group data were expressed as mean \pm SEM and statistical significance was set at the $P<0.05$ level. Subject characteristics were compared under supplementation groups using independent samples $t$-tests (Table 5.1). The exercise times to exhaustion during familiarisation, $\mathrm{T} 1$ and $\mathrm{T} 2$ were assessed using mixed-model repeated measures ANOVA (within subject factors: trials; between subject factor: supplementation groups) followed by simple main effect analysis. The remaining data, which contained multiple time points during each trial, were analysed using mixed-model repeated measures ANOVA (within subject factors: trial $\mathrm{x}$ time of sample; between subject factor: supplementation groups). Mauchly's test was consulted and Greenhouse-Geisser correction was applied if the assumption of sphericity was violated. If a significant $P$ value was identified for the three-way interaction effect (supplementation group $\mathrm{x}$ trial $\mathrm{x}$ time of sample), supplementation was deemed to have a significant effect. If a significant $P$ value was identified for the main effect of time (time of sample), multiple pairwise comparisons were made 
using Bonferonni confidence interval adjustment, with statistical significance set at $P<0.01$. 


\subsection{RESULTS}

Exercise times to exhaustion at $85 \% \dot{\mathrm{VO}}_{2} \max$ were similar between groups during familiarisation and T1 (PS, P: 8:02 $\pm 1: 37,7: 46 \pm 0: 41 ; 7: 51 \pm 1: 36,8: 09 \pm 0: 54$ min:s). A significant interaction effect (supplementation group $\mathrm{x}$ trial, $F=7.27$; $P=0.007$; partial eta $^{2}=0.85$ ) indicated that supplementation had a significant effect on time to exhaustion; post hoc analysis revealed no differences between trials for $\mathrm{P}$ $(P=0.670)$ and significant differences between trials for PS $(P=0.001)$. The magnitude of change in exercise times to exhaustion (individual T2 values minus $\mathrm{T} 1$ values $)$ in PS were 2:00 \pm 0:28 min:s while $P$ remained similar $(0: 07 \pm 0: 13$ min:s $)$ (Figure 5.3).

Heart Rates

Supplementation did not significantly affect last minute heart rates during the protocol (supplementation group $\mathrm{x}$ trial $\mathrm{x}$ time of sample, $P=0.058$ ). Mean last minute heart rates increased throughout the stages of the protocol (Table 5.2), being $119 \pm 2,137 \pm 3,156 \pm 3$ and $183 \pm 2$ beats $\cdot \min ^{-1}$, respectively at $45,55,65$ and $85 \% \dot{\mathrm{VO}}_{2} \max$. 


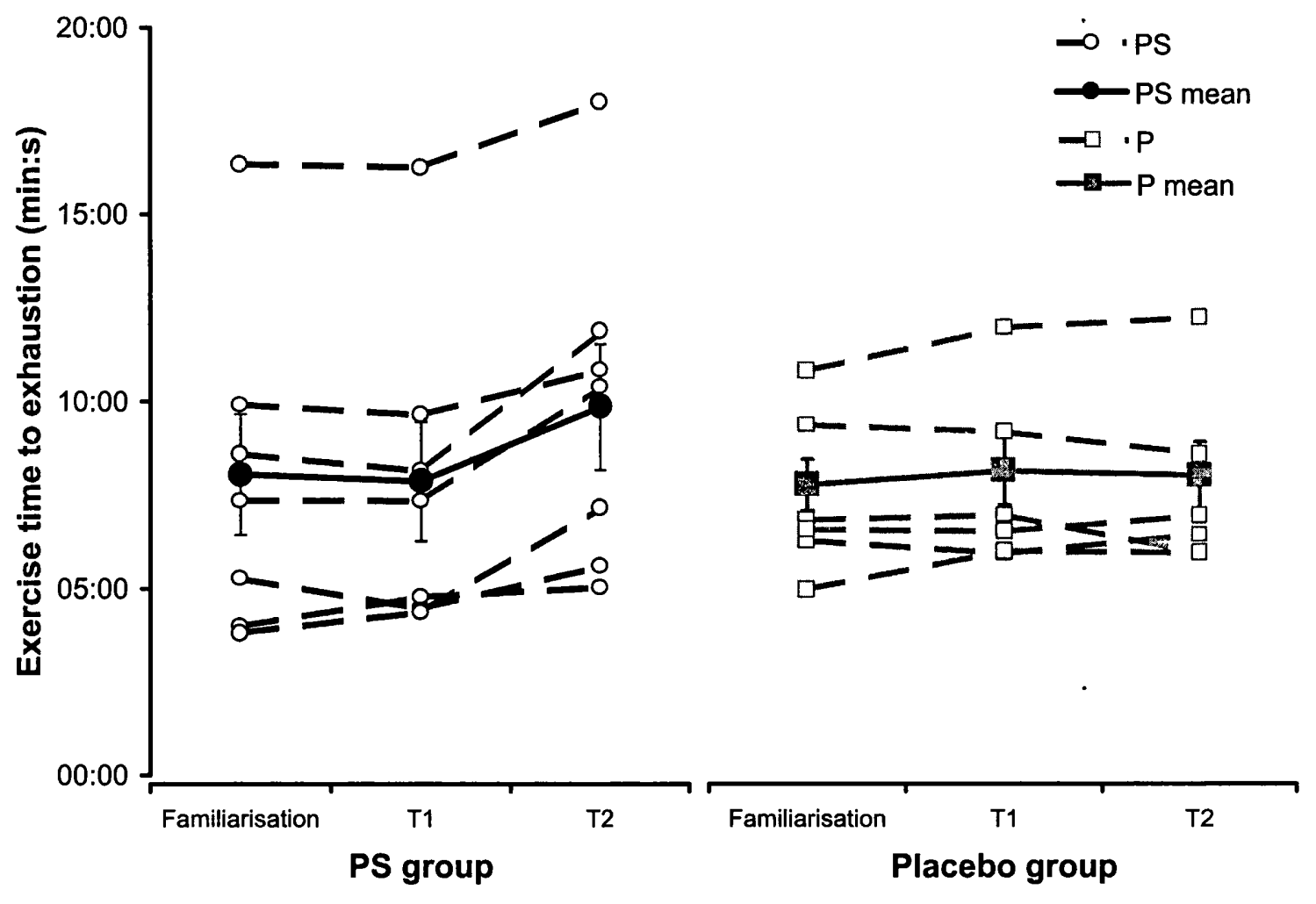

Figure 5.3 Exercise times to exhaustion at $85 \% \dot{\mathrm{VO}}_{2}$ max completed at the end of each exercise trial. Individual data are presented as open shapes and mean data are presented as filled shapes. PS: phosphatidylserine group; P: placebo group. (supplementation group $\mathrm{x}$ trial interaction, $P=0.007$ ). 


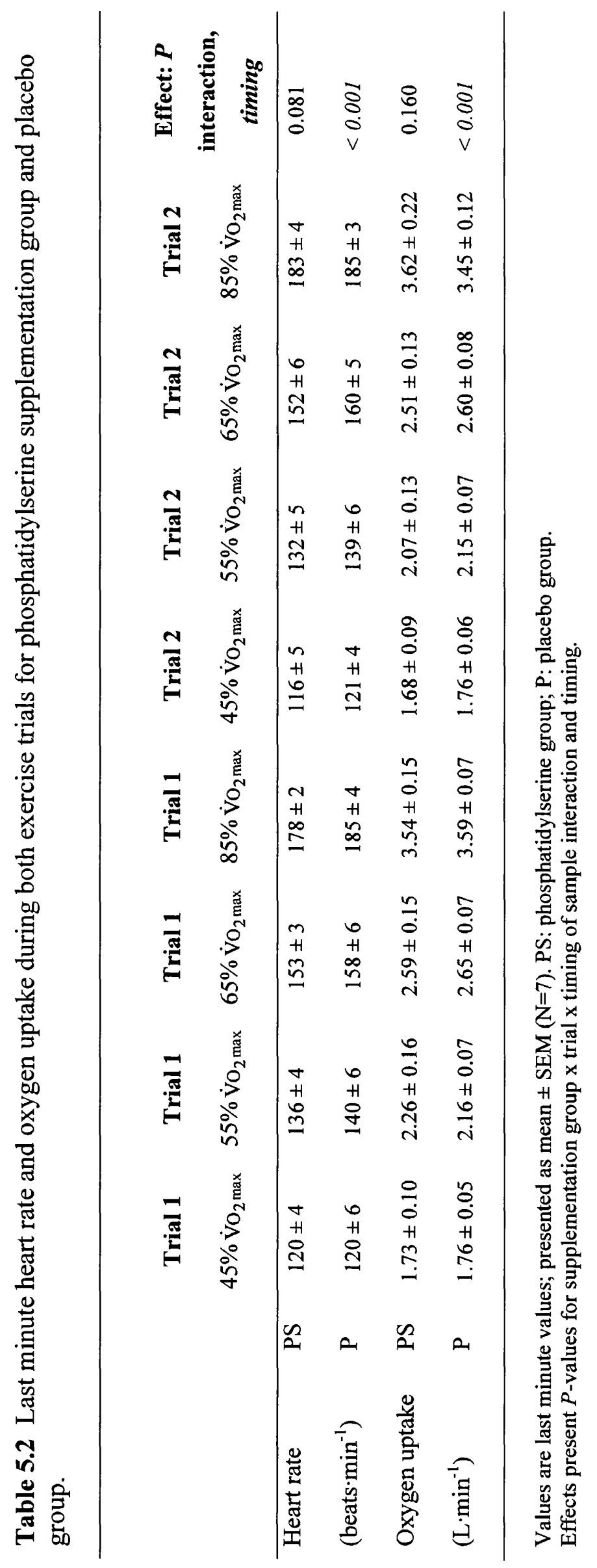


Last minute oxygen uptake data are presented in table 5.2. These data increased progressively with exercise intensity $\left(47 \pm 1,58 \pm 1,70 \pm 1\right.$ and $97 \pm 2 \% \dot{\mathrm{VO}}_{2} \max$; time of sample effect, $F=562.66 ; P<0.001 ;$ partial $_{\text {eta }}{ }^{2}=0.98$ ) and the supplement $\mathrm{x}$ trial $\mathrm{x}$ time of sample interaction was not significant $(P=0.160)$. Steady state was confirmed in all stages at exercise intensities up to and including $65 \% \quad \mathrm{~V}_{2}{ }_{2}$ max. Mean carbohydrate oxidation, fat oxidation and total energy expenditure were: 1.40 $\pm 0.06,1.95 \pm 0.11$, and $2.49 \pm 0.14 \mathrm{~g} \cdot \mathrm{min}^{-1} ; 0.28 \pm 0.02,0.27 \pm 0.02$, and $0.27 \pm$ $0.02 \mathrm{~g} \cdot \mathrm{min}^{-1} ; 34.0 \pm 0.8,42.3 \pm 1.0$, and $51.1 \pm 1.1 \mathrm{~kJ} \cdot \mathrm{min}^{-1}$, respectively at each exercise stage (Figure 5.4). Supplementation had no effect on carbohydrate oxidation (supplementation group $\mathrm{x}$ trial $\mathrm{x}$ time of sample, $P=0.596$ ), fat oxidation (supplementation group $\mathrm{x}$ trial $\mathrm{x}$ time of sample, $P=0.187$ ) or calculated energy expenditure (supplementation group $\mathrm{x}$ trial $\mathrm{x}$ time of sample, $P=0.595$ ).

\section{Oxygen Kinetic Analyses}

The mean response times for the on-kinetic response $\left(\mathrm{MRT}_{\text {on }}\right)$ were significantly

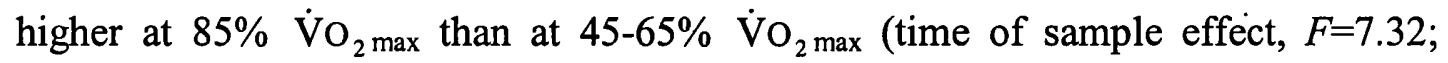
$P=0.019 ;$ partial $^{\text {eta }}{ }^{2}=0.38$ ); however, the three-way interaction was not significant $(P=0.069)$ (Figure 5.5). Supplementation had no effect on $\mathrm{MRT}_{\text {off }}$ (supplementation group $\mathrm{x}$ trial $\mathrm{x}$ time of sample, $P=0.449$ ), and exercise intensity had no significant effect (time of sample effect, $F=4.53 ; P=0.055 ;$ partial eta $^{2}=0.27$ ) (Figure 5.5). 
(a)

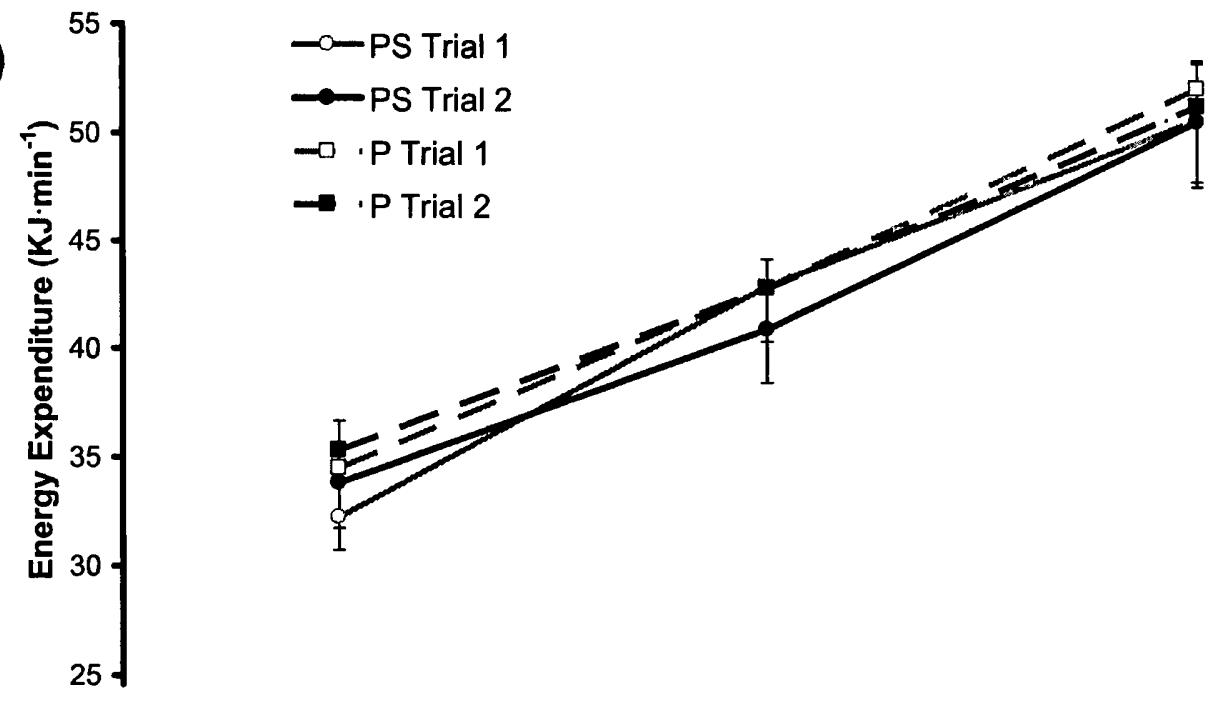

(b)

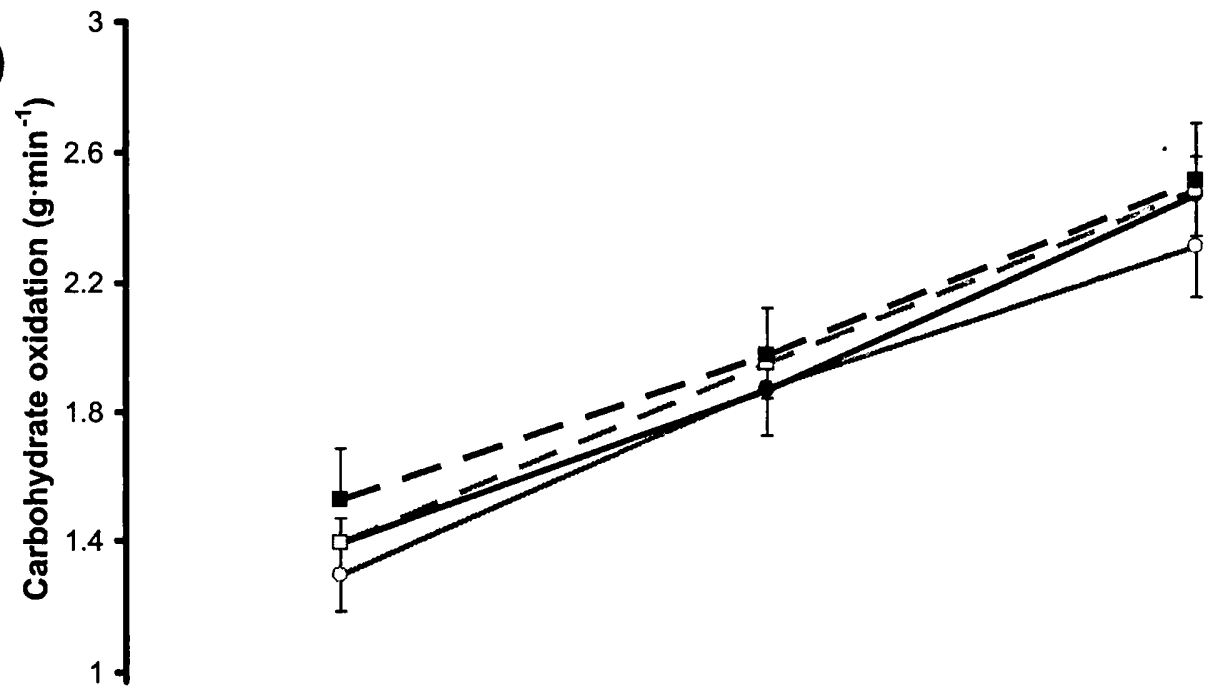

(c)

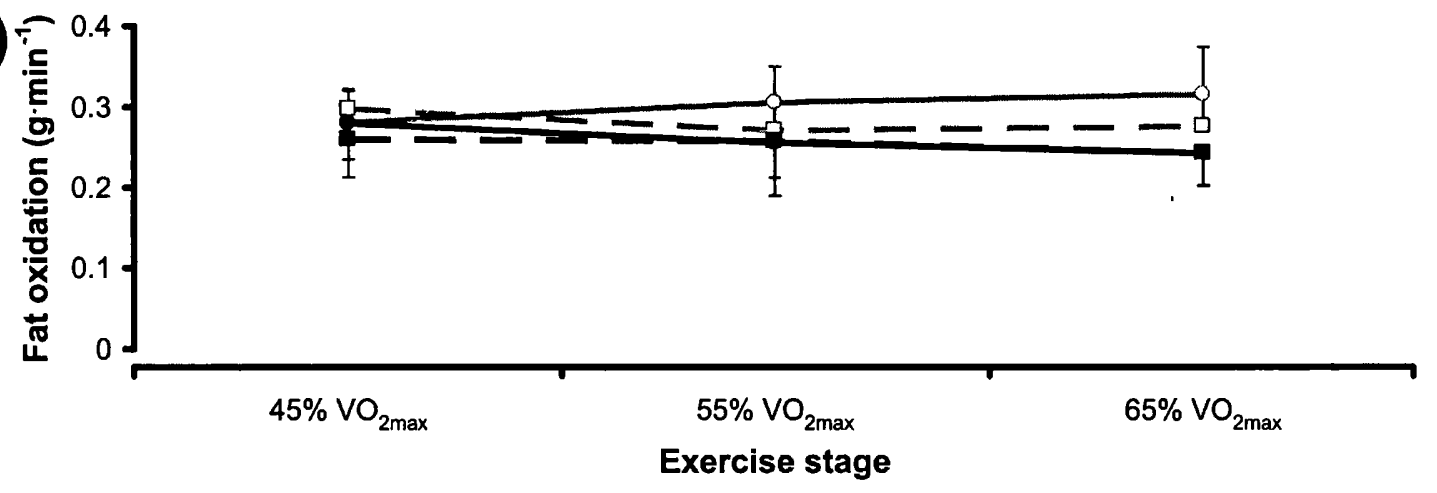

Figure 5.4 (a) Energy expenditure, (b) carbohydrate oxidation rates, and (c) fat oxidation rates during each main exercise trial. Values represent mean \pm SEM $(\mathrm{N}=7)$. PS: phosphatidylserine group; P: placebo group. Trial 1: presupplementation; Trial 2: post-supplementation. 

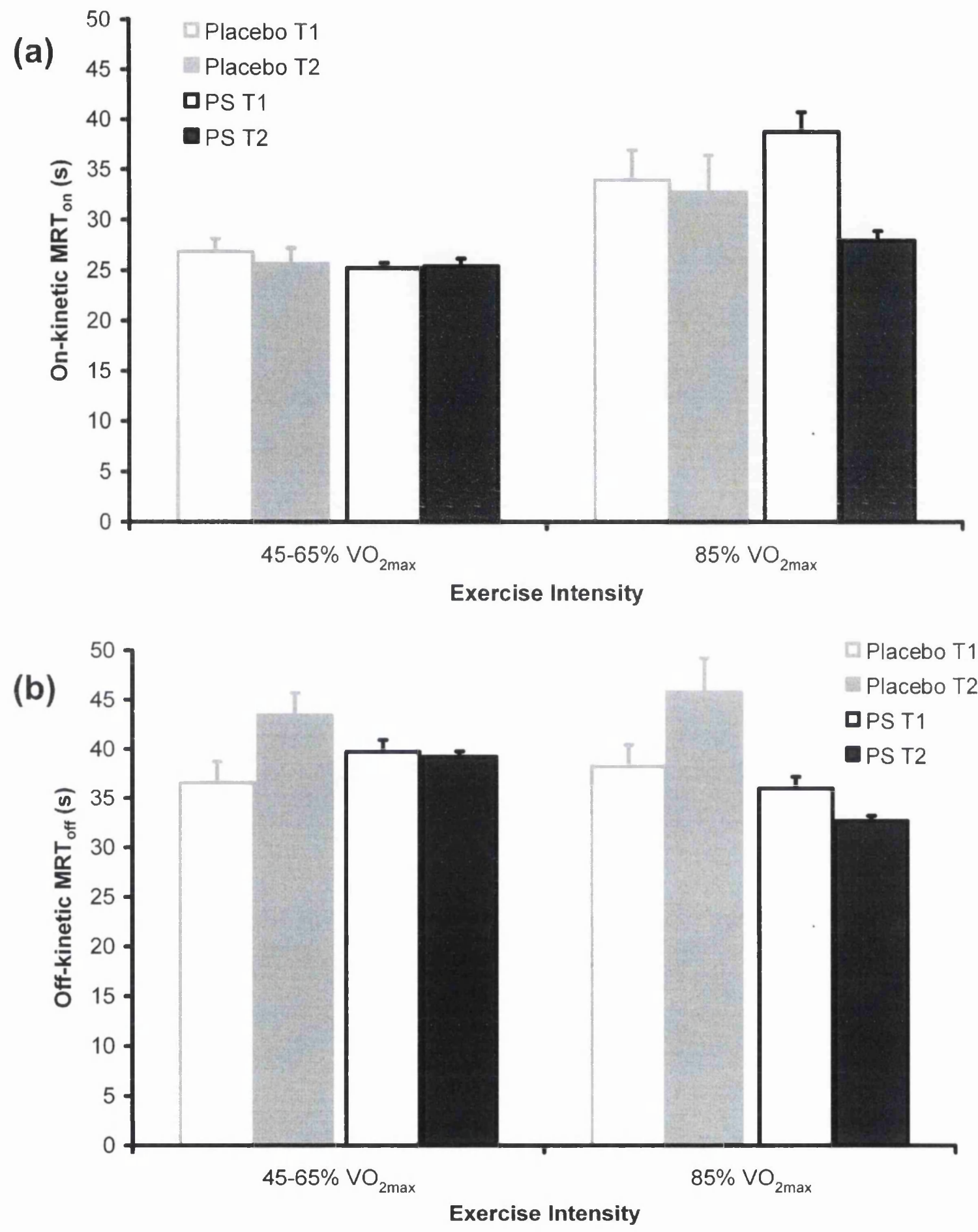

Figure 5.5 (a) On-kinetic mean response times and (b) off-kinetic mean response times during moderate and very heavy exercise stages. Values represent mean \pm SEM (N=7). PS: phosphatidylserine group; P: placebo group. Trial 1: presupplementation; Trial 2: post-supplementation. 
Estimated plasma volume fell by $7-11 \%$ during the first two exercise. stages and remained below Pre-exercise values throughout exercise (Table 5.3); supplementation had no effect (supplementation group $\mathrm{x}$ trial $\mathrm{x}$ time of sample, $P=0.392$ ) and no statistical differences were identified between $\mathrm{T} 1$ and $\mathrm{T} 2$ (trial effect: $P=0.491)$.

Blood Lactate and Glucose Concentrations

Blood lactate concentrations were significantly elevated from Pre-exercise values from Post-65\% (Table 5.4), and peaked at Post- $85 \%$ (mean value for all trials was $\left.7.70 \pm 0.33 \mathrm{mmol} \cdot \mathrm{L}^{-1}\right)$. Blood glucose concentrations did not differ significantly from Pre-exercise values at any time point (Table 5.4), and no differences were identified between groups or as a result of supplementation. 


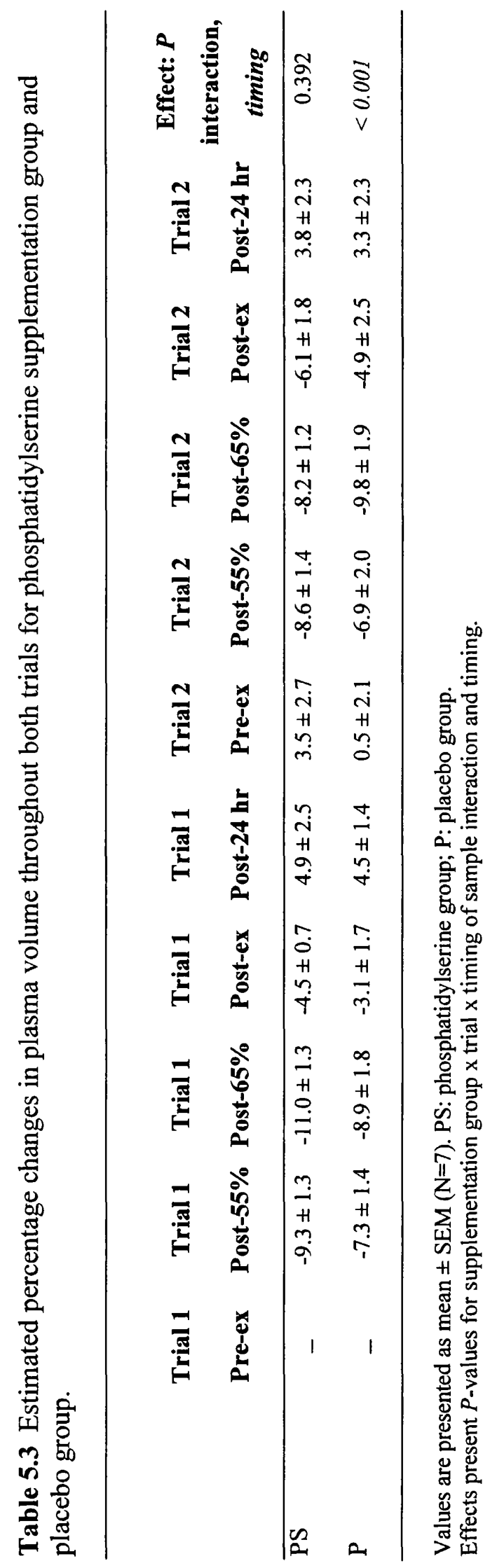




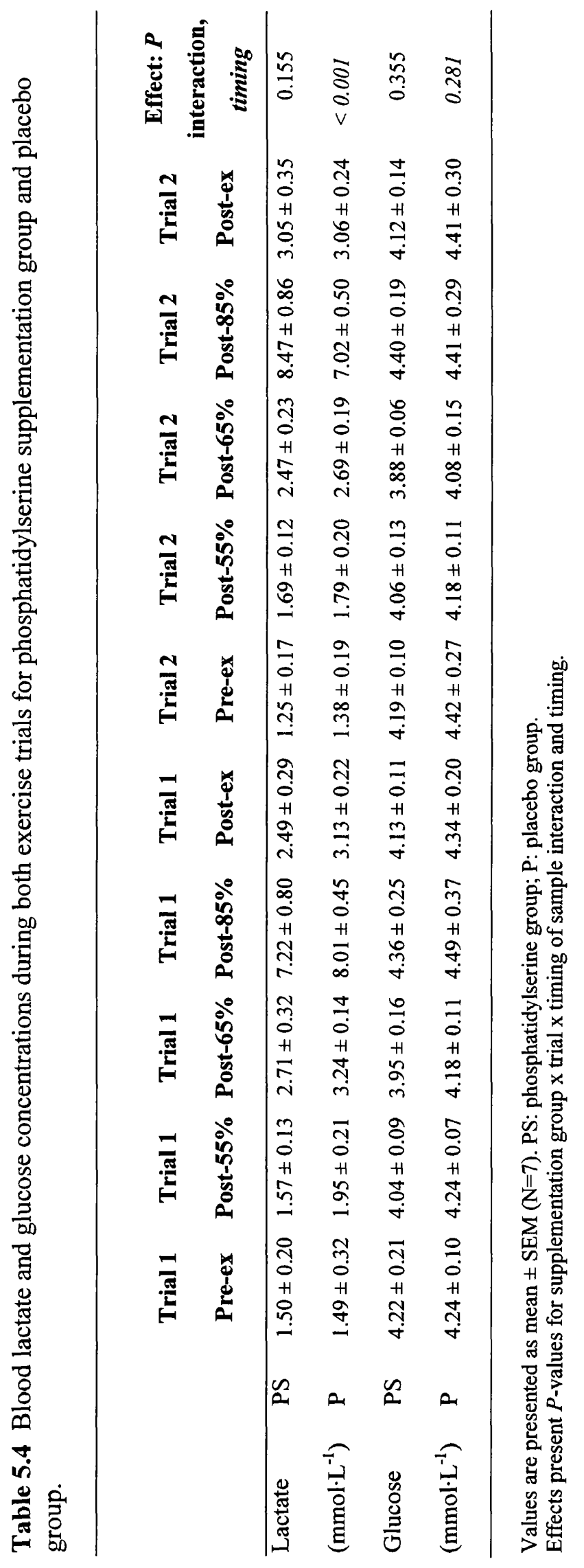


Serum cortisol concentrations were significantly elevated by exercise (time of sample effect, $F=20.69 ; P<0.001$; partial eta $^{2}=0.63$ ) from Pre-exercise values of 378 $\pm 21 \mathrm{nmol} \cdot \mathrm{L}^{-1}$ to Post-exercise values of $554 \pm 32 \mathrm{nmol} \cdot \mathrm{L}^{-1}$ (Figure 5.6). Serum cortisol concentrations were not significantly affected by supplementation (supplementation group $\mathrm{x}$ trial $\mathrm{x}$ time of sample, $P=0.118$ ).

\section{Feeling States}

Significant temporal changes were observed in all of the EFI subscales; subjects reported decreases in revitalisation, positive engagement, and tranquillity and increases in physical exhaustion through exercise (Table 5.5). The three-way interactions were not significant in any of the subscales (Table 5.5). 


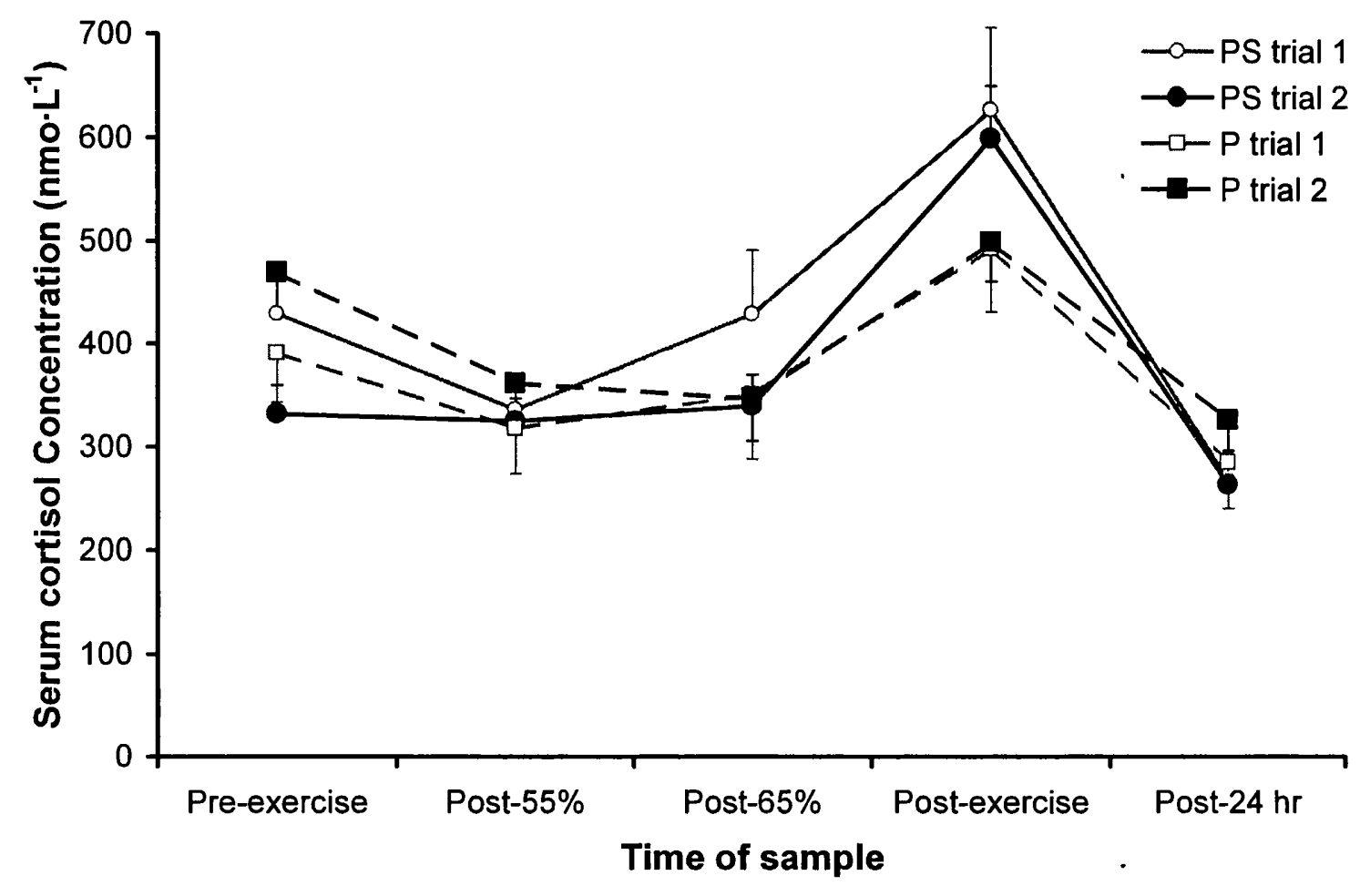

Figure 5.6 Serum cortisol concentrations throughout each trial. Values represent mean \pm SEM $(\mathrm{N}=8)$. Pre-P: trial prior to supplementation with placebo; $\mathrm{P}$ : trial supplemented with placebo; Pre-PS: trial prior to supplementation with phosphatidylserine; PS: trial supplemented with phosphatidylserine. 


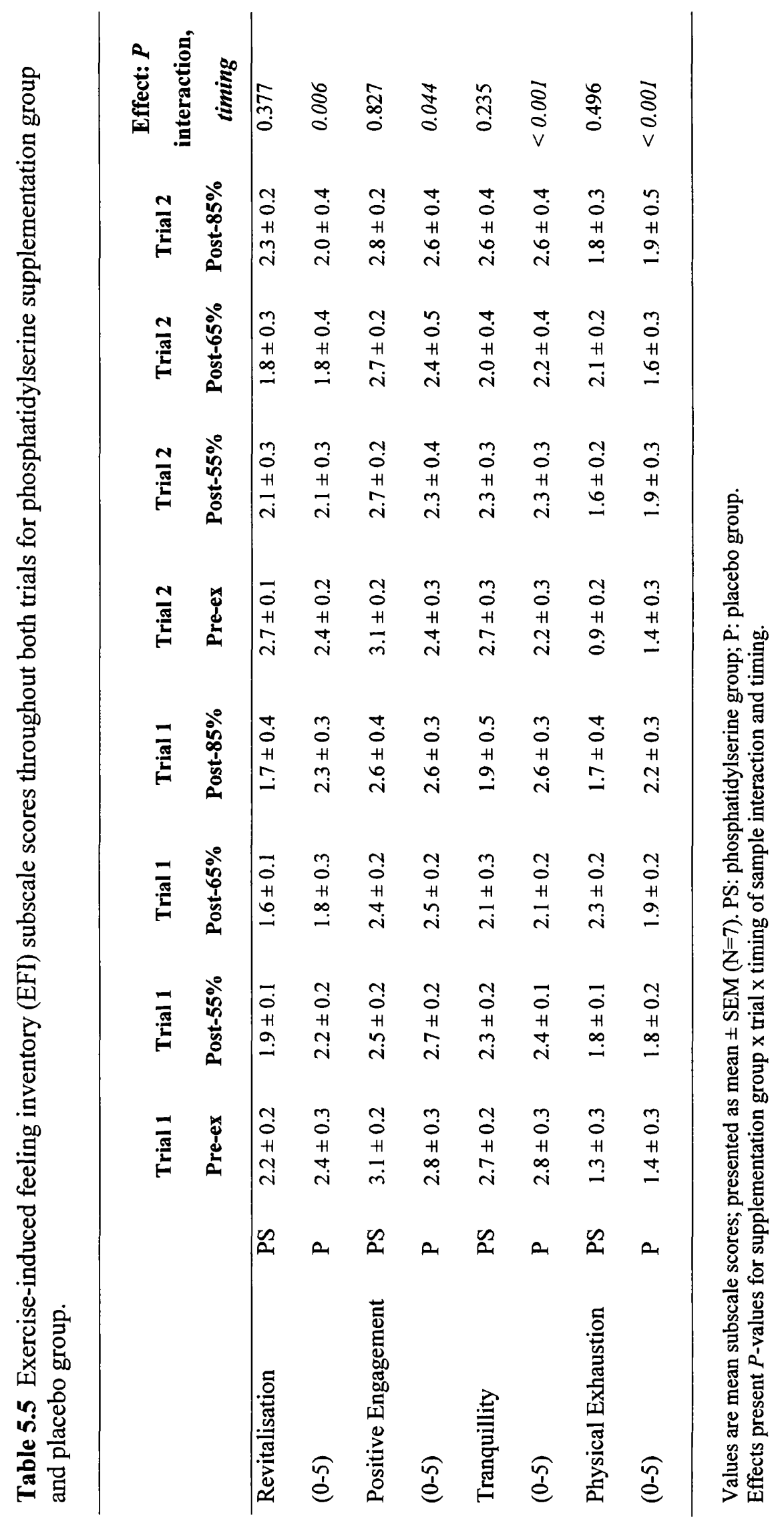




\subsection{DISCUSSION}

The primary finding of this investigation was that oral supplementation with 750 $\mathrm{mg} \cdot \mathrm{d}^{-1} \mathrm{~S}$-PtdSer for $10 \mathrm{~d}$ significantly affected exercise capacity in a group of recreationally active subjects during a staged intermittent cycling protocol. Furthermore, the enhancements in exercise capacity in PS ranged from 0:15 to 3:47 min:s, while the exercise times to exhaustion remained unchanged in P. .

It was originally hypothesised that PtdSer would influence the primary oxygen uptake kinetic response and thereby increase exercise capacity. However, supplementation did not significantly affect $\mathrm{MRT}_{\text {on }}$. Furthermore, no differences were observed between trial or supplementation group in $\mathrm{MRT}_{\text {off; }}$, therefore, the current data presents insufficient evidence to support any change in the primary oxygen uptake kinetic response following supplementation.

The causes of fatigue during cycling at $85 \% \mathrm{~V}_{2}{ }_{\text {max }}$ (within an intensity domain that has been previously classified as very heavy exercise (Ozyener et al., 2001)) have not been fully elucidated and might include central and peripheral components (Kent-Braun, 1999). Exercise during the final bout was associated with near maximal oxygen uptakes and heart rates in addition to relatively high blood lactate concentrations; therefore, it can be assumed that heavy demands were placed on both oxidative and non-oxidative phosphorylation. Metabolic acidosis has been implicated as a mechanism of peripheral fatigue either through direct effects on the contractile proteins or through inhibition of key regulatory enzymes such as phosphofructokinase (Cooke et al., 1988); however, it might be more likely that 
other ionic imbalances contribute to fatigue in this exercise model (Allen, 2004). Invitro studies have demonstrated that low concentrations of PtdSer are effective in activating $\left(\mathrm{Na}^{+}-\mathrm{K}^{+}\right)$-dependent ATPase in mammalian kidney (Specht and Robinson, 1973) and brain (Tsakiris and Deliconstantinos, 1984) preparations. Similarly, $\mathrm{Ca}^{2+}$ ATPase, an enzyme primarily responsible for $\mathrm{Ca}^{2+}$ re-uptake from the muscle cytosol into the sarcoplasmic reticulum, is known to require PtdSer (Morrot et al., 1990; Sepulveda and Mata, 2004). Therefore, it is plausible that exogenous S-PtdSer delayed the onset of fatigue by maintaining ionic homeostasis for longer during exercise.

In addition, Tibbits et al. (1981) reported that extended exercise training increased the levels of phospholipids, especially PtdSer content, in rat cardiac sarcolemma. This adaptation to training suggests that additional PtdSer within the heart muscle has functional benefits during exercise. An increase in membrane bound PtdSer might have the potential to enhance myocardial excitation-contraction coupling, potentially through the activation of different protein kinase $\mathrm{C}$ isoforms (Sugden and Bogoyevitch, 1995) and/or enhanced calcium uptake (Pepeu et al., 1996). Thus, it is plausible that these mechanisms also might have contributed to delaying fatigue in the present study. However, without corroborating data from further studies that investigate the in-vivo pharmacological actions of S-PtdSer the proposed mechanisms remain speculative.

The significant rise in serum cortisol concentration that followed the final bout of exercise suggested that the protocol activated the HPA axis (Monteleone et al., 1990). However, supplementation with PtdSer did not significantly influence serum 
cortisol concentrations (Figure 5.5). This finding does not concur with the results of Fahey and Pearl (1998) who found that S-PtdSer, using a similar supplementation regime, attenuated serum cortisol concentrations following resistance training. Furthermore, Monteleone et al. (1992) reported that $800 \mathrm{mg} \cdot \mathrm{d}^{-1} \mathrm{BC}-\mathrm{PtdSer}$ resulted in significant reductions in plasma cortisol and adrenocorticotrophic hormone $(\mathrm{ACTH})$ concentrations during submaximal cycle exercise in untrained subjects.

The elevation in blood cortisol is a generic response to stress from both psychological and physical origin; consequently, there is considerable interindividual variability in response to exercise. Although the choice of experimental design in the current study investigated individual changes in response (pre- to postsupplementation) and, therefore, reduced the possible effect of subject selection related bias, the possibility exists that the current dose might have been insufficient to attenuate the cortisol response in these active individuals. Alternatively, the current exercise protocol required that all participants continued the final exercise bout until exhaustion in both trials; therefore, it remains plausible that any effect of PtdSer supplementation on cortisol concentrations were masked as the PS group completed significantly more work in $\mathrm{T} 2$ when compared with $\mathrm{T} 1$.

Blood glucose concentrations remained unchanged throughout all trials. This finding was in agreement with previous studies using similar exercise protocols (Monteleone et al., 1990; Monteleone et al., 1992). The concomitant effects of reduced insulin and elevations in $\mathrm{ACTH}$, cortisol and adrenaline are responsible for controlling blood glucose during exercise (Åstrand and Rodahl, 1986). Therefore, any effects that S-PtdSer supplementation might have had on blood ACTH and 
cortisol did not appear to have over-challenged blood glucose homeostasis during exercise. Furthermore, the calculated rates of carbohydrate, fat and combined fuel oxidation during the steady state stages of exercise were similar in all trials, suggesting that any change in the HPA axis induced by S-PtdSer supplementation did not affect substrate oxidation during moderate exercise stages.

All subscales of the EFI were sensitive to change during the exercise. However, the three-way interaction did not reach significance in any subscale; therefore, there was no evidence to suggest that feeling states differed following supplementation in either supplementation group. The participants in the current study provided baseline responses that were similar to those of other recreationally active populations prior to exercise training (Lacaille et al., 2004), indicating that the testing procedures did not induce large changes in feeling states prior to exercise in these subjects. Benton et al. (2001) reported improvements in mood after mental stress within a sub-group of young healthy adults following short-term S-PtdSer supplementation. Nevertheless, these improvements were only identifiable in a subgroup of subjects who scored higher than the median for neuroticism; people who score highly on this dimension are known to display strong emotional reactions to stress (Benton et al., 2001). Consequently, the baseline emotional state of an individual might influence the efficacy of S-PtdSer in altering feeling states during exercise.

This is the first study to identify the ergogenic properties of phosphatidylserine; therefore, we suggest that further studies are required to substantiate these findings and to investigate the potential uses of S-PtdSer in exercise and physical activity. In 
addition, future studies are warranted to investigate the mechanism by which SPtdSer might act physiologically. 
CHAPTER SIX

\section{GENERAL DISCUSSION}




\section{GENERAL DISCUSSION}

The general aim of this research was to evaluate the effects of short-term oral supplementation with $750 \mathrm{mg} \cdot \mathrm{d}^{-1}$ soy derived phosphatidylserine (S-PtdSer) on exercise capacity, neuroendocrine function, inflammation, oxidative stress, muscle damage and perceived feeling states.

Results from the first experimental study (chapter 3) showed that S-PtdSer supplementation for $10 \mathrm{~d}$ prior to an exhaustive running protocol, consisting of intermittent running immediately followed by a progressive run, did not affect perceived muscle soreness or markers of muscle damage and lipid peroxidation. However, S-PtdSer tended to improve running performance. As the amount of exercise performed tended to be different between trials, it was difficult to evaluate the efficacy of S-PtdSer as an antioxidant under these exercise conditions. Consequently, the second experimental study (chapter 4) was designed to evaluate the effects of S-PtdSer supplementation on markers of muscle damage, inflammation and oxidative stress that follow downhill running. Additionally, the unexpected and potentially useful finding that S-PtdSer supplementation tended to improve performance led to the evolution of the third experimental study (chapter 5) which evaluated the potential ergogenic properties of S-PtdSer following intermittent cycling.

The second experimental study showed that moderate intensity downhill running led to elevations in markers of muscle damage (plasma myoglobin concentrations and creatine kinase activities), lipid peroxidation (serum hydroperoxide concentrations), 
inflammation (serum IL-6 concentrations) and DOMS (perceived soreness). However, the administration of $750 \mathrm{mg} \cdot \mathrm{d}^{-1} \mathrm{~S}-\mathrm{PtdSer}$ for $7 \mathrm{~d}$ prior to and continued until $2 \mathrm{~d}$ following exercise was not effective in attenuating any of the aforementioned markers in previously familiarised active males.

The main finding from experimental study 3 (chapter 5) was that $10 \mathrm{~d}$ of oral supplementation with S-PtdSer led to significant improvements in exercise capacity during a staged intermittent cycling protocol in recreationally active subjects. However, the mechanism(s) responsible for this finding were unclear as supplementation did not significantly affect oxygen kinetic mean response times, serum cortisol concentrations, substrate oxidation and feeling states during this study.

The findings from this series of experimental studies are collectively discussed in this chapter. Where appropriate, conclusions regarding current understanding are included and recommendations for future investigations aimed at evaluating the effects of phosphatidylserine supplementation on exercising humans are presented.

Effects of Phosphatidylserine Supplementation on Neuroendocrine Function

Experimental studies 1 and 2 demonstrated that prior supplementation with 750 $\mathrm{mg} \cdot \mathrm{d}^{-1}$ S-PtdSer for $10 \mathrm{~d}$ was not effective in attenuating serum cortisol concentrations after exhaustive exercise. During experimental study 1, intermittent running caused elevations in circulatory cortisol concentrations in university soccer 
players that were similar regardless of supplementation. Likewise, circulatory cortisol concentrations in active male subjects were elevated following the last bout of intermittent cycling (study 3); although, these concentrations were unaffected by supplementation. The increases in serum cortisol concentrations confirmed that the exercise protocols used in both of these experimental studies induced changes in the hypothalamo-pituitary-adrenal (HPA) axis (Monteleone et al., 1990).

Monteleone et al. (1992) reported that $800 \mathrm{mg} \cdot \mathrm{d}^{-1}$ of bovine cortex derived phosphatidylserine (BC-PtdSer) resulted in significant reductions in plasma cortisol and adrenocorticotrophic hormone (ACTH) concentrations during submaximal cycle exercise in untrained subjects. These equivocal findings might reflect the fact that the blood samples were taken following exhaustive exercise in experimental studies 1 and 3, while S-PtdSer supplementation increased exercise capacity during cycling and tended to improve performance during running; therefore, any effect that SPtdSer might have exerted on the HPA axis could have been masked. Alternatively, variations in these results might reflect the training status of the participants used in the respective studies, especially considering that trained subjects experience attenuated HPA alterations during exercise (Luger et al., 1987).

During experimental study 2, downhill running did not significantly influence plasma cortisol and ACTH concentrations; additionally, in agreement with the results from experimental studies 1 and 3 , supplementation did not significantly affect the concentrations of these hormones. Therefore, it is tempting to conclude that the source of PtdSer might influence the actions of this supplement on neuroendocrine function, and more specifically that S-PtdSer supplementation does 
not affect circulatory cortisol concentrations. However, Fahey and Pearl (1998) reported that $800 \mathrm{mg} \cdot \mathrm{d}^{-1} \mathrm{~S}-\mathrm{PtdSer}$ attenuated serum cortisol concentrations following intensive resistance training in weight-trained subjects.

There are several potential reasons for the discrepancy in findings between the current experimental data and those of Fahey and Pearl (1998). The study design used in experimental study 2 included pre-supplementation control trials that reduce the chance of type I error (incorrectly rejecting the null hypothesis), where as previous studies in this field (Monteleone et al., 1990; Monteleone et al., 1992; Fahey and Pearl, 1998) have employed simple cross-over study designs. Additionally, although Fahey and Pearl (1998) reported significant decreases in circulatory cortisol concentrations following exercise after treatment with S-PtdSer, there were no significant differences in serum cortisol concentrations between treatments or any of the resting trials. The completion of post-hoc analyses after non-significant main effects have been determined elevates the probability of type I error; therefore, differences in statistical procedures could have contributed to these equivocal findings. Also, it remains plausible that differences in the mode and intensity of exercise, which influence the HPA axis, might have contributed to differences in findings; the intensive resistance training programme required subjects to train as hard as possible using as much weight as possible, while the exercise protocol used in experimental study 2 required the subjects to run downhill at a relatively low metabolic demand.

Supplementation did not influence blood glucose concentrations in any of the experimental studies (chapters 3 - 5). This finding was in agreement with previous 
studies using submaximal cycling exercise protocols (Monteleone et al., 1990; Monteleone et al., 1992). The concomitant effects of a number of hormones (e.g., insulin, ACTH, cortisol and adrenaline) are responsible for controlling blood glucose during exercise (Åstrand and Rodahl, 1986). Therefore, even if S-PtdSer supplementation does influence neuroendocrine function, as suggested previously, there is no evidence to suggest that this level of supplementation influences blood glucose homeostasis during exercise. Furthermore, the calculated carbohydrate and fat oxidation rates using non-protein respiratory exchange ratios during the steady state stages of cycling were not influenced by supplementation in experimental study 3. Thus, any change in the HPA axis, induced by S-PtdSer supplementation, did not affect substrate oxidation during moderate exercise intensities. Although no data from these experimental studies, or other published research, are available at present, it remains possible that S-PtdSer supplementation might influence substrate utilisation at higher exercise intensities.

In conclusion, the effects of S-PtdSer supplementation on neuroendocrine function remain equivocal. The possibility exists that the dose employed during the current experimental studies might have been insufficient to attenuate the cortisol response in these active individuals. Consequently, the optimal dose of S-PtdSer might vary with the training status of the participants. 
Data from experimental studies 1 and 2 showed that short-term supplementation with $750 \mathrm{mg} \cdot \mathrm{d}^{-1} \mathrm{~S}-\mathrm{PtdSer}$ did not effectively reduce muscle damage, as measured by creatine kinase activities and myoglobin concentrations in the circulation, in well accustomed active participants following intermittent running designed to simulate soccer match play (study 1) or downhill running (study 2). These findings are in agreement with Fahey and Pearl (1998) who reported that similar doses of S-PtdSer did not influence serum creatine kinase activities after heavy resistance training. Together, these data suggest that S-PtdSer supplementation does not directly influence the structural integrity of the muscle cell in well-accustomed participants.

The exercise protocols undertaken during these experimental studies caused increases in perceived muscle soreness; furthermore, the magnitude of reported soreness was similar during these trials and the temporal patterns were consistent with delayed onset of muscle soreness. However, supplementation did not affect perceived soreness during either experimental study. This finding could reflect the subjective nature of reporting perceived muscle soreness and the unknown variance in this measure is a limitation to the research.

In living cell membranes, PtdSer is mainly located within the cytosolic monolayer (Devaux, 1991). This membrane asymmetry is actively maintained and is believed to be vital for proper cell function (Voelker, 1989). The externalisation of PtdSer is thought to signal apoptosis (programmed cell death) and initiate cell phagocytosis (Tyurina et al., 2000). It has been suggested that unfamiliar eccentric muscle activity 
causes mechanical damage to the most susceptible muscle fibres (Friden et al., 1983), which become subsequently removed through phagocytosis. Therefore, as the subjects used in experimental studies 1 and 3 completed familiarisation trials the level of structural muscle damage caused by the subsequent exercise trials was probably reduced. It is possible that S-PtdSer supplementation might be effective in reducing muscle damage in susceptible cells (i.e., when individuals have not completed prior familiarisation trials).

Effects of Phosphatidylserine Supplementation on Exercise-Induced Oxidative Stress

Experimental study 1 investigated the effects of $10-\mathrm{d}$ oral supplementation with 750 $\mathrm{mg} \cdot \mathrm{d}^{-1} \mathrm{~S}-\mathrm{PtdSer}$ on oxidative stress following exhaustive intermittent running in active young males. Although supplementation had no effect on lipid peroxidation (serum hydroperoxide concentrations) or resistance to oxidation (serum conjugated diene lag times), supplementation with S-PtdSer tended to improve performance. Consequently, it remained unclear if this supplementation regime was ineffective in enhancing the defences against exercise induced oxidative stress or if the completion of more work had led to the production of higher amounts of reactive oxygen species that had overwhelmed the combined defences.

With the findings from study 1 in mind, the subsequent experimental study (study 2) was designed to evaluate the effects of short-term oral supplementation on oxidative stress caused by repeatable amounts of downhill treadmill running in active young males. It was hypothesised that the eccentric components of downhill running would 
cause muscle damage and lead to the development of an acute inflammatory response (Childs et al., 2001). The acute inflammatory response has been previously shown to elevate free radical production and activate cytokines (Macintyre et al., 1995). Free radicals can lead to oxidative damage in a wide range of molecular structures where the antioxidant defences are overwhelmed. In addition, exercise induced erythrocyte haemolysis has the potential to elevate loosely bound iron in extracellular fluids and tissues (Jenkins et al., 1993). Therefore, downhill treadmill running has the potential to induce oxidative stress.

As expected, the downhill running protocol used in experimental study 2 caused significant increases in lipid peroxidation (as measured by serum hydroperoxide concentrations), demonstrating that exercise led to elevations in free radical production that overwhelmed the antioxidant defences and increased oxidative stress. However, lipid peroxidation, susceptibility to LDL oxidation (as measured by changes in conjugated diene concentrations) and resistance to LDL oxidation (as measured by $t_{1 / 2 \max }$ LDL oxidation) were similar following supplementation with $\mathrm{S}$ PtdSer and placebo. Therefore, no evidence was available to suggest that exogenous S-PtdSer was able to enhance antioxidant defences under these conditions.

The findings from experimental studies 1 and 2 showed that supplementation with S-PtdSer transiently increased plasma $\gamma$-tocopherol. Increases in plasma and tissue $\gamma-$ tocopherol in rats have been demonstrated to attenuate protein and ascorbate oxidation following inflammation-mediated damage (Jiang et al., 2002). Thus the increases in plasma $\gamma$-tocopherol concentrations and probable increases in tissue $\gamma$ tocopherol concentrations might have augmented the antioxidant defence, although 
supplementation had no effect on concentrations of the other plasma antioxidant vitamins (vitamin $\mathrm{C}, \alpha$-tocopherol, retinol and $\beta$-carotene), lipid peroxidation or susceptibility of LDL to in-vitro oxidation during either experimental study.

\section{Effects of Phosphatidylserine Supplementation on Immune Function}

Downhill running was confirmed to elevate interleukin-6 (IL-6) concentrations in plasma during experimental study 2 . Increases in pro-inflammatory cytokines, such

as IL-6, are characteristic of an acute inflammatory response (Childs et al., 2001). Although consistent with other downhill running studies (e.g., Thompson et al., 2004; Peake et al., 2005), the magnitude of increase in IL-6 (2-3 times baseline values) was relatively modest when compared with the changes that have been reported to occur following exercise that is associated with higher metabolic demands, such as prolonged intermittent running (Thompson et al., 2001b) and prolonged endurance running (Suzuki et al., 2000).

Supplementation with S-PtdSer did not affect serum IL-6 concentrations after downhill running. It is possible that the magnitude of increase in IL-6 was insufficient to reveal any effect of supplementation. Alternatively, as the mode, intensity and duration of exercise appear to influence the immune response to exercise (Vassilakopoulos et al., 2005), the lack of supplementation effect on circulatory IL-6 might be specific to eccentric exercise. The stimulus and/or mechanism of IL-6 induction during extended concentric exercise are probably different; therefore, it remains plausible, although speculative, that exogenous S- 
PtdSer could moderate IL-6 concentrations following other types of exercise. In support of this suggestion, vitamin $\mathrm{C}$ supplement (a recognised antioxidant) has been demonstrated to moderate IL-6 concentrations following prolonged shuttle running (Thompson et al., 2001b) but not downhill running (Thompson et al., 2004).

Effects of Phosphatidylserine Supplementation on Subjective Feelings During and Following Exercise

Short-term S-PtdSer supplementation has been previously reported to improve subjective well-being during two weeks of intense resistance training (Fahey and Pearl, 1998). Although the mechanism(s) behind this finding were not clear, these results might reflect an action of S-PtdSer to attenuate the exercise induced HPA axis. As cortisol has the potential to stimulate amino acid release from tissues (such as skeletal muscle), these authors hypothesised that S-PtdSer might have decreased protein degradation and consequently improved perceived well-being during this period of overtraining. In support of this hypothesis, they found that supplementation with S-PtdSer also reduced perceived muscular soreness when compared with placebo. However, markers of protein degradation were not quantified during this study to confirm that this was in fact the case. Also, supplementation did not affect serum creatine kinase activities. As increases in circulatory creatine kinase activity are accepted as an indirect marker of structural muscle damage, empirical data does not support this theory. 
An alternative explanation for the positive effect of supplementation on perceived muscle soreness and well-being could be that S-PtdSer positively influenced higher brain functions and moderated subjective feelings. As described in Chapter 1, PtdSer is an important cofactor that has the potential to influence higher brain functions. Exogenous PtdSer has been demonstrated to enhance cognitive function (Blokland et al., 1999), improve learning/memory (Sakai et al., 1996; Furushiro et al., 1997; Alves et al., 2000; Suzuki et al., 2001) and attenuate the effects of stress (Drago et al., 1991), in rodents. Additionally, PtdSer supplementation has been demonstrated to exert therapeutic actions on higher brain functions in human patients with cognitive decline (Amaducci and SMID group, 1988; Crook et al., 1992; Cenacchi et al., 1993). Furthermore, Benton et al. (2001) demonstrated that short-term SPtdSer supplementation in a sub-group of healthy young males reduced feelings of stress and improved mood in the response to a stressful mathematical challenge.

Supplementation did not influence the ratings of perceived exertion during experimental study 1 . However, this measure can only be employed during exercise. Also, this exercise protocol involved maximal sprinting during part A, and part $\mathrm{B}$ was continued until volitional fatigue; consequently, perceived exertion was unlikely to be affected by supplementation. Therefore, an exercise specific measure of exercise-induced feeling states (EFI) (Gauvin and Rejeski, 1993) was used in experimental studies 2 and 3 in order to assess the effects of S-PtdSer supplementation on feeling states (revitalisation, positive engagement, tranquillity, physical exhaustion) before and after exercise. 
Although feeling states were not significantly altered by downhill running during study 2, exhaustive intermittent cycling influenced all of the measured feeling states (study 3). Furthermore, supplementation did not significantly influence any of the measured feeling states in experimental study 2 or study 3 . The small changes in feeling states recorded in study 2 probably reflected the low metabolic demand during the downhill exercise protocol and might have contributed to the lack of supplementation effect in this study. As the neuroendocrine responses to mental stress and physical stress are comparable and considering that S-PtdSer significantly enhanced exercise capacity during experimental study 3 , it is possible that any effect of supplementation on feeling states might have been obscured. Additionally, as the variance in reporting the subjective feeling states was not quantified in this research, this limitation might have hidden any effect of supplementation.

Therefore, the effects of S-PtdSer on feeling states during exercise are unclear; consequently, future research could evaluate the effect of S-PtdSer supplementation on feeling states and decision-making during other types of exercise.

\section{Ergogenic Effects of Phosphatidylserine Supplementation}

Unexpectedly, experimental study 1 demonstrated a tendency for S-PtdSer supplementation to improve sprint and exercise performances during intermittent running when compared with a placebo. These findings led to experimental study 3 , which hypothesised that PtdSer supplementation could reduce accumulated oxygen deficit and improve exercise tolerance, as PtdSer has the potential to influence 
mitochondrial enzymatic activity and contribute to the maintenance of ionic homeostasis. Although these data did not support any change in the primary oxygen uptake kinetic response following supplementation, supplementation did affect exercise times to exhaustion at $85 \% \dot{\mathrm{VO}}_{2}$ max. Following supplementation, the PS group ( $750 \mathrm{mg} \cdot \mathrm{d}^{-1}$ of S-PtdSer for $10 \mathrm{~d}$ ) increased times to exhaustion by $29 \pm 8 \%$, while the exercise times to exhaustion for the placebo group did not differ $(-1 \pm 3 \%)$ following supplementation with a glucose placebo.

The final bout of cycling elicited near maximal oxygen uptakes and heart rates in addition to relatively high blood lactate concentrations. The causes of fatigue during exercise that places high demands on aerobic and anaerobic phosphorylation have not been fully elucidated and might include central and peripheral components (Kent-Braun, 1999). Metabolic acidosis has been implicated as a mechanism of peripheral fatigue, either through direct effects on the contractile proteins or through inhibition of key regulatory enzymes such as phosphofructokinase (Cooke et al., 1988); however, other ionic imbalances might be more likely contribute to fatigue in this exercise model (Allen, 2004). In-vitro studies have demonstrated that PtdSer is effective in activating $\left(\mathrm{Na}^{+}-\mathrm{K}^{+}\right)$-dependent ATPase in mammalian tissue preparations (Specht and Robinson, 1973; Tsakiris and Deliconstantinos, 1984). Similarly, $\mathrm{Ca}^{2+}$-ATPase, an enzyme primarily responsible for $\mathrm{Ca}^{2+}$ re-uptake from the muscle cytosol into the sarcoplasmic reticulum, is known to require PtdSer (Morrot et al., 1990; Sepulveda and Mata, 2004). Therefore, it is plausible that exogenous S-PtdSer delayed the onset of fatigue by maintaining ionic homeostasis for longer during exercise. However, without corroborating data from further studies 
that investigate the in-vivo pharmacological actions of S-PtdSer, the preceding mechanism remains speculative.

The finding that S-PtdSer might possess ergogenic properties is of potential interest to the athletic population, especially those that undertake intermittent and/or high intensity exercise. However, these are the only published data to evaluate the ergogenic properties of PtdSer and the findings are based on relatively small numbers of moderately trained subjects; therefore, further research is warranted.

\section{Directions for Future Research}

The placebo controlled experimental studies described within this thesis (chapters 3 to 5) compared the effects of a PtdSer supplement (soybean lecithin enriched with SPtdSer) with those of a glucose placebo. Therefore, it is important to point out that the findings presented here relate to the activity of this particular supplement. In order that future research can evaluate the effects of S-PtdSer supplementation, it is envisaged that further experiments could use a placebo containing soybean lecithin that is devoid of S-PtdSer rather than a glucose placebo.

Although it is likely that the dose of S-PtdSer provided in this series of experimental studies will have caused transient increases in the PtdSer content within systemic circulation and incorporation of PtdSer within cell membranes, the biokinetics of PtdSer following oral administration in humans are largely unknown. Future studies are required to quantify circulatory and tissue concentrations of PtdSer in animal and 
human models following exogenous PS supplementation. Earlier in this chapter it was proposed that the dose employed in the current studies might have been insufficient to modify neuroendocrine function in these relatively active individuals; consequently, the optimal dose of S-PtdSer could vary between participants. It is envisaged that this research would help identify factors that might affect S-PtdSer availability and uptake (i.e., quality of supplement, interaction effects with other nutrients, training status, age, dietary habits, etc.).

The findings from experimental study 2 suggest that supplementation with PS does not influence muscle damage, muscle soreness, inflammation or oxidative stress following eccentric muscle activity in well accustomed young males. It remains possible that exogenous S-PtdSer supplementation might protect susceptible cells; therefore, the act of familiarisation might have negated any prophylactic effects of PtdSer supplementation in the present study. Consequently, future research could investigate the effects of S-PtdSer supplementation in naive subjects (subjects that have not completed familiarisation trials).

A variety of animal studies have confirmed anti-inflammatory functions of PtdSer; however, the findings from experimental study 2 did not show any effect of supplementation on plasma IL-6 concentrations following eccentric exercise that was associated with a low metabolic challenge. It would be of interest to evaluate the effects of S-PtdSer supplementation on immunological function following prolonged exercise, which has been demonstrated to cause a larger immunological challenge. 
The findings from experimental studies 1 and 3 suggest that S-PtdSer possess ergogenic properties. However, these findings are specific to the subject populations and the type of activities undertaken. Therefore, further research is warranted to identify whether these findings are transferable to other athletic and/or clinical populations. In addition, the specific mechanism(s) responsible for the ergogenic actions of S-PtdSer are currently speculative; consequently, there is potential to design and undertake experiments that specifically investigate both peripheral and central mechanism that might explain these findings. 


\section{GENERAL CONCLUSIONS}

With reference to the specific supplementation regimes, exercise protocols and measurement techniques used in this series of experiments, the following general conclusions can be made:

1. The results from experimental study 1 suggested that oral S-PtdSer supplementation tends to improve exercise performance. Although the findings from study 3 confirmed potential ergogenic properties of this supplement, supplementation did not significantly affect oxygen kinetics, neuroendocrine function or fuel oxidation rates. Therefore the mechanism(s) responsible for these findings remain unclear.

2. The results from all experimental studies suggested that oral S-PtdSer supplementation does not influence the effects of exercise on hypothalamopituitary-adrenal axis in active young males.

3. The results from experimental studies 1 and 2 suggested that oral S-PtdSer supplementation does not affect lipid peroxidation, muscle damage, and muscle soreness following prolonged exhaustive running and downhill running. However, oral S-PtdSer supplementation does cause a transient increase in the concentration of $\gamma$-tocopherol, an established plasma antioxidant vitamin. 
4. The results from experimental study 2 suggested that oral S-PtdSer supplementation does not influence inflammation caused by downhill running, as measured by circulatory concentrations of the pro-inflammatory cytokine interleukin-6.

5. The results from experimental study 2 and 3 suggested that oral S-PtdSer supplementation does not significantly change feeling states during, immediately after and on the day following exercise.

6. No adverse reactions to oral S-PtdSer supplementation were reported during this series of experimental studies. Furthermore, no data are presented to suggest negative effects of supplementation. 


\section{REFERENCES}

Allen, D. G. (2004). Skeletal muscle function: role of ionic changes in fatigue, damage and disease. Clinical and Experimental Pharmacology and Physiology. 31 485-493.

Alves, C. S., Andreatini, R., da Cunha, C., Tufik, S. and Vital, M. A. (2000). Phosphatidylserine reverses reserpine-induced amnesia. European Journal of Pharmacology. 404 161-167.

Amaducci, L. and SMID group. (1988). Phosphatidylserine in the treatment of Alzheimer's Disease: results of a multicenter study. Psychopharmacology Bulletin. 24 130-134.

Aoi, W., Naito, Y., Takanami, Y., Kawai, Y., Sakuma, K., Ichikawa, H., Yoshida, N. and Yoshikawa, T. (2004). Oxidative stress and delayed-onset muscle damage after exercise. Free Radical Biology and Medicine. 37 480-487.

Aramaki, Y., Matsuno, R., Nitta, F., Arima, H. and Tsuchiya, S. (1997). Negatively charged liposomes inhibit tyrosine phosphorylation of 41-kDa protein in murine macrophages stimulated with LPS. Biochemical and Biophysical Research Communications. 231 827-830.

Armstrong, R. B., Warren, G. L. and Warren, J. A. (1991). Mechanisms of exerciseinduced muscle fibre injury. Sports Medicine. 12 184-207.

Åstrand, P.-O. and Rodahl, K. (1986). Textbook of Work Physiology, Physiological Bases of Exercise, Third Edition. McGraw-Hill International Editions. pp 556-562.

Benton, D., Donohoe, R. T., Sillance, B. and Nabb, S. (2001). The influence of phosphatidylserine supplementation on mood and heart rate when faced with an acute stressor. Nutritional Neuroscience. 4 169-178. 
Bick, R. J., Buja, L. M., Van Winkle, W. B. and Taffet, G. E. (1998). Membrane asymmetry in isolated canine cardiac sarcoplasmic reticulum: comparison with skeletal muscle sarcoplasmic reticulum. Journal of Membrane Biology. 164 169175.

Bigon, E., Boarato, E., Bruni, A., Leon, A. and Toffano, G. (1979). Pharmacological effects of phosphatidylserine liposomes: regulation of gylcolysis and energy level in brain. British Journal of Pharmacology. 66 167-174.

Blokland, A., Honig, W., Browns, F. and Jolles, J. (1999). Cognition-enhancing properties of subchronic phosphatidylserine (PS) treatment in middle-aged rats: comparison of bovine cortex PS with egg PS and soybean PS. Nutrition. 15 778-783.

Borg, G. (1998). Borg's Perceived Exertion and Pain Scales. Champaign, IL: Human Kinetics.

Bruni, A., Mietto, L., Secchi, F. E. and Chizzolini, C. (1994). Lipid mediators of immune reactions: effect of a linked phosphorylserine group. Immunology Letters. 42 87-90.

Bruni, A., Orlando, P., Mietto, L. and Viola, G. (1992). Phospholipid metabolism in rat intestinal mucosa after oral administration of lysophospholipids. In Neurobiology of Essential Fatty Acids (edited by N. G. Bazan), pp. 243-249. New York: Plenum Press

Bruni, A., Toffano, G., Leon, A. and Boarato, E. (1976). Pharmacological effects of phosphatidylserine liposomes. Nature. 260 331-333.

Casamenti, F., Mantovani, P., Amaducci, L. and Pepeu, G. (1979). Effect of phosphatidylserine on acetylcholine output from the cerebral cortex of the rat. Journal of Neurochemistry. 32 529-533.

Casamenti, F., Scali, C. and Pepeu, G. (1991). Phosphatidylserine reverses the agedependent decrease in cortical acetylcholine release: a microdialysis study.

European Journal of Pharmacology. 194 11-16. 
Catignani, G. L. and Bieri, J. G. (1983). Simultaneous determination of retinol and alpha-tocopherol in serum or plasma by liquid chromatography. Clinical Chemistry. 29 708-712.

Cenacchi, T., Baggio, C. and Palin, E. (1987). Human tolerability of oral phosphatidylserine assessed through laboratory examinations. Clinical Trials Journal. 21 125-130.

Cenacchi, T., Bertoldin, T., Farina, C., Fiori, M. G. and Crepaldi, G. (1993). Cognitive decline in the elderly: a double-blind, placebo-controlled multicenter study on efficacy of phosphatidylserine administration. Aging. 5 123-133.

Childs, A., Jacobs, C., Kaminski, T., Halliwell, B. and Leeuwenburgh, C. (2001). Supplementation with vitamin $\mathrm{C}$ and $\mathrm{N}$-acetyl-cysteine increases oxidative stress in humans after an acute muscle injury induced by eccentric exercise. Free Radical Biology and Medicine. 31 745-753.

Clarkson, P. M., Nosaka, K. and Braun, B. (1992). Muscle function after exerciseinduced muscle damage and rapid adaptation. Medicine and Science in Sports and Exercise. 24 512-520.

Cooke, R., Franks, K., Luciani, G. B. and Pate, E. (1988). The inhibition of rabbit skeletal muscle contraction by hydrogen ions and phosphate. Journal of Physiology. 395 77-97.

Cooney, R. V., Custer, L. J., Okinaka, L. and Franke, A. A. (2001). Effects of dietary sesame seeds on plasma tocopherol levels. Nutrition and Cancer. 39 66-71.

Crook, T., Petrie, W., Wells, C. and Massari, D. C. (1992). Effects of phosphatidylserine in Alzheimer's disease. Psychopharmacology Bulletin. 28 61-66.

Dacaranhe, C. D. and Terao, J. (2001). A unique antioxidant activity of phosphatidylserine on iron-induced lipid peroxidation of phospholipid bilayers. Lipids. 36 1105-1110. 
Dekkers, J. C., Van Dooran, L. J. P. and Kemper, H. C. G. (1996). The role of antioxidant vitamins and enzymes in the prevention of exercise-induced muscle damage. Sports Medicine. 21 213-238.

Devaux, P. F. (1991). Static and dynamic lipid asymetry in cell membranes.

Biochemistry. 30 1163-1173.

Diagne, A., Fauvel, J., Record, M., Chap, H. and Douste-Blazy, L. (1984). Studies on ether phospholipids. II. Comparative composition of various tissues from human, rat and guinea pig. Biochimica et Biophysica Acta. 793 221-231.

Dill, D. B. and Costill, D. L. (1974). Calculation of percentage changes in volumes of blood, plasma and red cells in dehydration. Journal of Applied Physiology. 37 247-248.

Drago, F., Spadaro, F., D'Agata, V., Valerio, C., Grassi, M., Mauceri, F., Pennisi, G. and Scapagnini, U. (1991). Protective action of phosphatidylserine on stress-induced behavioral and autonomic changes in aged rats. Neurobiology of Aging. 12 437-440.

Facci, L., Cusinato, F., Negro, A., Monastra, G., Signorelli, A. and Bruni, A. (2000). The immunosuppressant steroid cholesterylphosphoserine inhibits tumour necrosis factor-alpha secretion in vitro and in vivo. Cytokine. 12 770-773.

Fahey, T. D. and Pearl, M. S. (1998). The hormonal and perceptive effects of phosphatidylserine administration during two weeks of resistive exercise-induced overtraining. Biology of Sport. 15 135-144.

Feasson, L., Stockholm, D., Freyssenet, D. and add, t. (2002). Molecular adaptations of neuromuscular disease-associated proteins in response to eccentric exercise in human skeletal muscle. Journal of Physiology. 543 297-306.

Folch, J. and Schneider, H. A. (1941). An amino acid constituent of ox brain cephalin. Journal of Biological Chemistry. 137 51-62.

Friden, J., Sjostrom, M. and Ekblom, B. (1983). Myofibrillar damage following intense eccentric exercise in man. International Journal of Sports Medicine. 4 170176. 
Furushiro, M., Suzuki, S., Shishido, Y., Sakai, M., Yamatoya, H., Kudo, S., Hashimoto, S. and Yokokura, T. (1997). Effects of oral administration of soybean lecithin transphosphatidylated phosphatidylserine on impaired learning of passive avoidance in mice. Japanese Journal of Pharmacology. 75 447-450.

Gauvin, L. and Rejeski, W. J. (1993). The Exercise-Induced Feeling Inventory: development and initial validation. Journal of Sport and Exercise Psychology. 15 403-423.

Ghosh, S., Xie, W. Q., Quest, A. F., Mabrouk, G. M., Strum, J. C. and Bell, R. M. (1994). The cysteine-rich region of raf-1 kinase contains zinc, translocates to liposomes, and is adjacent to a segment that binds GTP-ras. Journal of Biological Chemistry. 269 10000-10007.

Giannesini, B., Cozzone, P. J. and Bendahan, D. (2003). Non-invasive investigations of muscular fatigue: metabolic and electromyographic components. Biochimie. 85 873-883.

Gilbreath, M. J., Hoover, D. L., Alving, C. R., Swartz, G. M. and Meltzer, M. S. (1986). Inhibition of lymphokine-induced macrophage microbicidal activity against Leishmania major by liposomes: characterization of the physicochemical requirements for liposome inhibition. Journal of Immunology. 137 1681-1687.

Gorski, J., Zendzian-Piotrowska, M., de Jong, Y. F., Niklinska, W. and Glatz, J. F. C. (1999). Effect of endurance training on the phospholipid content of skeletal muscles in the rat. European Journal of Applied Physiology. 79 421-425.

Goth, A., Adams, H. R. and Knoohuizen, M. (1971). Phosphatidylserine: selective enhancer of histamine release. Science. 173 1034-1035.

Grassi, B. (2000). Skeletal muscle VO2 on-kinetics: set by $\mathrm{O} 2$ delivery or by $\mathrm{O} 2$ utilization? New insights into an old issue. Medicine and Science in Sports and Exercise. 32 108-116. 
Heikinheimo, L. and Somerharju, P. (2002). Translocation of pyrene-labeled phosphatidylserine from the plasma membrane to mitochondria diminishes systematically with molecular hydrophobicity: implications on the maintenance of high phosphatidylserine content in the inner leaflet of the plasma membrane. Biochimica et Biophysica Acta - Molecular Cell Research. 1591 75-85.

Hensley, K., Fedynyshyn, J., Ferrell, S., Floyd, R. A., Gordon, B., Grammas, P., Hamdheydari, L., Mhatre, M., Mou, S. and Pye et, al. (2003). Message and proteinlevel elevation of tumor necrosis factor alpha (TNF alpha) and TNF alphamodulating cytokines in spinal cords of the G93A-SOD1 mouse model for amyotrophic lateral sclerosis. Neurobiology of Disease. 14 74-80.

Hoffmann, P. R., Kench, J. A., Vondracek, A., Kruk, E., Daleke, D. L., Jordan, M., Marrack, P., Henson, P. M. and Fadok, V. A. (2005). Interaction between phosphatidylserine and the phosphatidylserine receptor inhibits immune responses in vivo. Journal of Immunology. 174 1393-1404.

Hubley-Kozey, C. L. (1991). Testing flexibility. In Physiological testing of the highperformance athlete (edited by J. A. MacDougall, H. A. Wenger and H. J. Green), pp. 309-359. Champaign, IL: Human Kinetics

Huynh, M. L., Fadok, V. A. and henson, P. M. (2002). Phosphatidylserinedependent ingestion of apoptotic cells promotes TGF- $\beta 1$ secretion and the resolution of inflammation. Journal of Clinical Investigation. 109 41-50.

International Society for the Advancement of Kinanthropometry (2001). International Standards for Anthropometric Assessment. Australia: The International Society for the Advancement of Kinanthropometry.

Jenkins, R. R., Krause, K. and Schofield, L. S. (1993). Influence of exercise on clearance of oxidant stress products and loosely bound iron. Medicine and Science in Sports and Exercise. 25 213-217. 
Jiang, Q. and Ames, B. N. (2003). Gamma-tocopherol, but not alpha-tocopherol, decreases proinflammatory eicosanoids and inflammation damage in rats. FASEB Journal: official publication of the Federation of American Societies for Experimental Biology. 17 816-822.

Jiang, Q., Lykkesfeldt, J., Shigenaga, M. K., Shigeno, E. T., Christen, S. and Ames, B. N. (2002). [gamma]-tocopherol supplementation inhibits protein nitration and ascorbate oxidation in rats with inflammation. Free Radical Biology and Medicine. 33 1534-1542.

Jorissen, B. L., Brouns, F., Van Boxtel, M. P. and Riedel, W. J. (2002). Safety of soy-derived phosphatidylserine in elderly people. Nutritional Neuroscience. $5337-$ 343.

Kajikawa, N., Kaibuchi, K., Matsubara, T., Kikkawa, U., Takai, Y., Nishizuka, Y., Itoh, K. and Tomioka, C. (1983). A possible role of protein kinase $\mathrm{C}$ in signalinduced lysosomal enzyme release. Biochemical and Biophysical Research Communications. 116 743-750.

Kanter, M. M., lesmes, G. R., Kaminsky, L. A., Ham-Saeger, J. L. and Nequin, N. D. (1988). Serum creatine kinase and lactate dehydrogenase changes following an eighty kilometre race. European Journal of Applied Physiology. 57 60-63.

Kendall, B. and Eston, R. (2002). Exercise-Induced Muscle Damage and the Potential Protective Role of Estrogen. Sports Medicine. 32 103-123.

Kent-Braun, J. A. (1999). Central and peripheral contributions to muscle fatigue in humans during sustained maximal effort. European Journal of Applied Physiology. 80 57-63.

Kim, H. Y., Akbar, M., Lau, A. and Edsall, L. (2000). Inhibition of neuronal apoptosis by docosahexaenoic acid (22:6n-3). Role of phosphatidylserine in antiapoptotic effect. Journal of Biological Chemistry. 275 35215-35223. 
Koutoku, T., Takahashi, H., Tomonaga, S., Oikawa, D., Saito, S., Tachibana, T., Han, L., Hayamizu, K., Denbow, D. M. and Furuse et, a. (2005). Central administration of phosphatidylserine attenuates isolation stress-induced behavior in chicks. Neurochemistry International. 47 183-189.

Kuge, O., Nishijima, M. and Akamatsu, Y. (1986). Phosphatidylserine biosynthesis in cultured Chinese hamster ovary cells. II. Isolation and characterization of phosphatidylserine auxotrophs. Journal of Biological Chemistry. 261 579.0-5794.

LaCaille, R. A., Masters, K. S. and Heath, E. M. (2004). Effects of cognitive strategy and exercise setting on running performance, perceived exertion, affect, and satisfaction. Psychology of Sport and Exercise. 5 461-476.

Latorraca, S., Piersanti, P., Tesco, G., Piacentini, S., Amaducci, L. and Sorbi, S. (1993). Effect of phosphatidylserine on free radical susceptibility in human diploid fibroblasts. Journal of Neural Transmission. Parkinson's Disease and Dementia Section. 6 73-77.

Liang, M. T., Meneses, P., Glonek, T., Kopp, S. J., Paulson, D. J., Schwartz, F. N. and Gierke, L. W. (1993). Effects of exercise training and anabolic steroids on plantaris and soleus phospholipids: a 31P nuclear magnetic resonance study. International Journal of Biochemistry. 25 337-347.

Lou, P., Gutman, R. L., Mao, F. W. and Greenspan, P. (1994). Effects of phosphatidylserine on the oxidation of low density lipoprotein. International Journal of Biochemistry. 26 539-545.

Luger, A., Deuster, P. A., Kyle, S. B., Gallucci, W. T., Montgomery, L. C., Gold, P. W., Loriaux, D. L. and Chrousos, G. P. (1987). Acute hypothalamic-pituitaryadrenal responses to the stress of treadmill exercise. Physiologic adaptations to physical training. New England Journal of Medicine. 316 1309-1315.

MacIntyre, D. L., Reid, D. W. and McKenzie, D. C. (1995). Delayed muscle soreness: the inflamation response to muscle injury and its clinical implications. Sports Medicine. 20 24-40. 
McDowell, I. F., McEneny, J. and Trimble, E. R. (1995). A rapid method for measurement of the susceptibility to oxidation of low-density lipoprotein. Annals of Clinical Biochemistry. 32 (2) 167-174.

McEneny, J., Trimble, E. R. and Young, I. S. (1998). A simple method for assessing copper-mediated oxidation of very-low-density lipoprotein isolated by rapid ultracentrifugation. Annals of Clinical Biochemistry. 35 (4) 504-514.

Miller, P. C., Bailey, S. P., Barnes, M. E., Derr, S. J. and Hall, E. E. (2004). The effects of protease supplementation on skeletal muscle function and DOMS following downhill running. Journal of Sports Sciences. 22 365-372.

Monastra, G. and Bruni, A. (1992). Decreased serum level of tumor necrosis factor in animals treated with lipopolysaccharide and liposomes containing phosphatidylserine. Lymphokine Cytokine Research. 11 39-43.

Monastra, G., Pege, G., Zanoni, R., Toffano, G. and Bruni, A. (1991). Lysophosphatidylserine-induced activation of mast cells in mice. Journal of Lipid Mediators. 3 39-50.

Mongar, J. L. and Svec, P. (1972). The effect of phospholipids on anaphylactic histamine release. British Journal of Pharmacology. 46 741-752.

Monteleone, P., Beinat, L., Tanzillo, C., Maj, M. and Kemali, D. (1990). Effects of phosphatidylserine on the neuroendocrine response to physical stress in humans. Neuroendocrinology. 52 243-248.

Monteleone, P., Maj, M., Beinat, L., Natale, M. and Kemali, D. (1992). Blunting by chronic phosphatidylserine administration of the stress-induced activation of the hypothalamo-pituitary-adrenal axis in healthy men. European Journal of Clinical Pharmacology. 42 385-388.

Moodley, I. and Mongar, J. L. (1981). IgG receptors on the mast cells. Agents and Actions. 11 77-83. 
Morrot, G., Zachowski, A. and Devaux, P. F. (1990). Partial purification and characterisation of the human erythrocyte. American Journal of Physiology. $21682-$ 86.

Nagai, Y., Aoki, J., Sato, T., Amano, K., Matsuda, Y., Arai, H. and Inoue, K. (1999). An alternative splicing form of phosphatidylserine-specific phospholipase A1 that exhibits lysophosphatidylserine-specific lysophospholipase activity in humans. Journal of Biological Chemistry. 274 11053-11059.

Nicholas, C. W., Nuttall, F. E. and Williams, C. (2000). The Loughborough Intermittent Shuttle Test: a field test that simulates the activity pattern of soccer. Journal of Sports Sciences. 18 97-104.

Nishijima, M., Kuge, O. and Akamatsu, Y. (1986). Phosphatidylserine biosynthesis in cultured Chinese hamster ovary cells. I. Inhibition of de novo phosphatidylserine biosynthesis by exogenous phosphatidylserine and its efficient incorporation. Journal of Biological Chemistry. 261 5784-5789.

Ozyener, F., Rossiter, H. B., Ward, S. A. and Whipp, B. J. (2001). Influence of exercise intensity on the on- and off-transient kinetics of pulmonary oxygen uptake in humans. Journal of Physiology. 533 891-902.

Palatini, P., Viola, G., Bigon, E., Menegus, A. M. and Bruni, A. (1991).

Pharmacokinetic characterization of phosphatidylserine liposomes in the rat. British Journal of Pharmacology. 102 345-350.

Peake, J. M., Suzuki, K., Wilson, G., Hordern, M., Nosaka, K., Mackinnon, L. and Coombes, J. S. (2005). Exercise-induced muscle damage, plasma cytokines, and markers of neutrophil activation. Medicine and Science in Sports and Exercise. 37 737-745.

Pepeu, G., Giovannelli, L., Giovannini, M. G. and Pedata, F. (1986). Effect of phosphatidylserine on cortical acetylcholine release and calcium uptake in adult and aging rats. In Phospholipid research and the nervous system. Biochemical and molecular pharmacology (edited by L. A. Horrocks, L. Freysz and G. Toffano), pp. 265-271. Padova: Liviana Press 
Pepeu, G., Pepeu, I. M. and Amaducci, L. (1996). A review of phosphatidylserine pharmacological and clinical effects. Is phosphatidylserine a drug for the ageing brain? Pharmacological Research. 33 73-80.

Peronnet, F. and Massicotte, D. (1991). Table of nonprotein respiratory quotient: an update. Canadian Journal of Sport Sciences. 16 23-29.

Ponzin, D., Mancini, C., Toffano, G., Bruni, A. and Doria, G. (1989).

Phosphatidylserine-induced modulation of the immune response in mice: effect of intravenous administration. Immunopharmacology. 18 167-176.

Ramsbottom, R., Brewer, J. and Williams, C. (1988). A progressive shuttle run test to estimate maximal oxygen uptake. British Journal of Sports Medicine. 22 141-144.

Rossiter, H. B., Howe, F. A., Ward, S. A., Kowalchuk, J. M., Griffiths, J. R. and Whipp, B. J. (2000). Intersample fluctuations in phosphocreatine concentration determined by 31P-magnetic resonance spectroscopy and parameter estimation of metabolic responses to exercise in humans. Journal of Physiology. 528 359-369.

Sakai, M., Yamatoya, H. and Kudo, S. (1996). Pharmacological effects of phosphatidylserine enzymatically synthesized from soybean lecithin on brain functions in rodents. Journal of Nutritional Science and Vitaminology. 42 47-54.

Schneider, D. A., Wing, A. N. and Morris, N. R. (2002). Oxygen uptake and heart rate kinetics during heavy exercise: a comparison between arm cranking and leg cycling. European Journal of Applied Physiology. 88 100-106.

Sepulveda, M. R. and Mata, A. M. (2004). The interaction of ethanol with reconstituted synaptosomal plasma membrane $\mathrm{Ca} 2+-\mathrm{ATPase}$. Biochimica et Biophysica Acta (BBA) - Biomembranes. 1665 75-80.

Shores, A. J. and Mongar, J. L. (1980). Modulation of histamine secretion from Concanavalin A-activated rat mast cells by phosphatidyl serine, calcium, cAMP, $\mathrm{pH}$ and metabolic inhibitors. Agents and Actions. 10 131-137.

Singer, S. J. and Nicolson, G. L. (1972). The fluid mosaic model of the structure of cell membranes. Science. 175 720-731. 
Smith, L. L., McCammon, M., Smith, S., Chamness, M., Israel, R. G. and O'Brien, K. F. (1989). White blood cell response to uphill walking and downhill jogging at similar metabolic loads. European Journal of Applied Physiology and Occupational Physiology. 58 833-837.

Soares, J. C. and Gershon, S. (1994). Advances in the pharmacotherapy of Alzheimer's disease. European Archives of Psychiatry and Clinical Neuroscience. 244 261-271.

Specht, S. C. and Robinson, J. D. (1973). Stimulation of the $\left(\mathrm{Na}^{+}+\mathrm{K}^{+}\right)$-dependent adenosine triphosphatase by amino acids and phosphatidylserine: chelation of trace metal inhibitors. Archives of Biochemistry and Biophysics. 154 314-323.

Sugden, P. H. and Bogoyevitch, M. A. (1995). Intracellular signalling through protein kinases in the heart. Cardiovascular Research. 30 478-492.

Sumikawa, K., Mu, Z., Inoue, T., Okochi, T. and Yoshida, T. (1993). Changes in erythrocyte membrane phospholipid composition induced by physical training and exercise. European Journal of Applied Physiology. 67 132-137.

Suzuki, K., Totsuka, M., Nakaji, S., Yamada, M., Kudoh, S., Liu, Q., Sugawara, K., Yamaya, K. and Sato, K. (1999). Endurance exercise causes interaction among stress hormones, cytokines, neutrophil dynamics, and muscle damage. Journal of Applied Physiology. 87 1360-1367.

Suzuki, K., Yamada, M., Kurakake, S., Okamura, N., Yamaya, K., Liu, Q., Kudoh, S., Kowatari, K., Nakaji, S. and Sugawara, e. a. (2000). Circulating cytokines and hormones with immunosuppressive but neutrophil-priming potentials rise after endurance exercise in humans. European Journal of Applied Physiology. 81 281287.

Suzuki, S., Yamatoya, H., Sakai, M., Kataoka, A., Furushiro, M. and Kudo, S. (2001). Oral administration of soybean lecithin transphosphatidylated phosphatidylserine improves memory impairment in aged rats. Journal of Nutrition. $1312951-2956$. 
Suzuki, T. T. and Kanfer, J. N. (1985). Purification and properties of an ethanolamine-serine base exchange enzyme of rat brain microsomes. Journal of Biological Chemistry. 260 1394-1399.

Takai, Y., Kishimoto, A., Iwasa, Y., Kawahara, Y., Mori, T. and Nishizuka, Y. (1979). Calcium-dependent activation of a multifunctional protein kinase by membrane phospholipids. Journal of Biological Chemistry. 254 3692-3695.

Tanaka, Y. and Schroit, A. J. (1983). Insertion of fluorescent phosphatidylserine into the plasma membrane of red blood cells. Journal of Biological Chemistry. 258 11335-11343.

Thompson, D., Bailey, D. M., Hill, J., Hurst, T., Powell, J. R. and Williams, C. (2004). Prolonged vitamin C supplementation and recovery from eccentric exercise. European Journal of Applied Physiology. 92 133-138.

Thompson, D., Williams, C., Kingsley, M., Nicholas, C. W., Lakomy, H. K., McArdle, F. and Jackson, M. J. (2001a). Muscle soreness and damage parameters after prolonged intermittent shuttle-running following acute vitamin $\mathrm{C}$ supplementation. International Journal of Sports Medicine. 22 68-75.

Thompson, D., Williams, C., McGregor, S. J., Nicholas, C. W., McArdle, F., Jackson, M. J. and Powell, J. R. (2001b). Prolonged vitamin C supplementation and recovery from demanding exercise. International Journal of Sport Nutrition and Exercise Metabolism. 11 466-481.

Thurnham, D. I., Smith, E. and Flora, P. S. (1988). Concurrent liquidchromatographic assay of retinol, alpha-tocopherol, beta-carotene, alpha-carotene, lycopene, and beta-cryptoxanthin in plasma, with tocopherol acetate as internal standard. Clinical Chemistry. 34 377-381.

Tibbits, G. F., Nagatomo, T., Sasaki, M. and Barnard, R. J. (1981). Cardiac sarcolemma: compositional adaptation to exercise. Science. 213 1271-1273.

Tidball, J. G. (2005). Inflammatory processes in muscle injury and repair. American Journal of Physiology. Regularory, Integrative and Comparative Physiology. 288 345-353. 
Toffano, G. and Bruni, A. (1980). Pharmacological properties of phospholipid liposomes. Pharmacological Research Communications. 12 829-845.

Toffano, G., Leon, A., Benvegnu, D., Boarato, E. and Azzone, G. F. (1976). Effect of brain cortex phospholipids on catechol-amine content of mouse brain.

Pharmacological Research Communications. 8 581-590.

Tsakiris, S. and Deliconstantinos, G. (1984). Influence of phosphatidylserine on $\left(\mathrm{Na}^{+}+\mathrm{K}^{+}\right)$-stimulated ATPase and acetylcholinesterase activities in dog brain synaptosomal plasma membranes. Biochemical Journal. 220 301-307.

Tyurina, Y. Y., Shvedova, A. A., Kawai, K., Tyurin, V. A., Komminineni, C., Quinn, P. J., Schor, N. F., Fabisiak, J. P. and Kagan, V. E. (2000). Phospholipid signaling in apoptosis: peroxidation and externalization of phophatidylserine. Toxicology. 148 93-101.

Van Loon, L. J., Greenhaff, P. L., Constantin-Teodosiu, D., Saris, W. H. and Wagenmakers, A. J. (2001). The effects of increasing exercise intensity on muscle fuel utilisation in humans. Journal of Physiology. 536 295-304.

Vance, J. E. and Steenbergen, R. (2005). Metabolism and functions of phosphatidylserine. Progress in Lipid Research. 44 207-234.

Vassilakopoulos, T., Roussos, C. and Spyros, Z. (2005). When are antioxidants effective in blunting the cytokine response to exercise? Medicine and Science in Sports and Exercise. 37 342-343.

Vincent, P., Sargueil, F., Sturbois-Balcerzak, B., Cassagne, C. and Moreau, P. (2001). Phosphatidylserine increase in rat liver endomembranes during the acute phase response. Biochimie. 83 957-960.

Voelker, D. R. (1989). Phosphatidylserine translocation to the mitochondrion is an ATP-dependent process in permeabilized animal cells. Proceedings of the National Academy of Sciences of the United States of America. 86 9921-9925. 
Voelker, D. R. and Frazier, J. L. (1986). Isolation and characterization of a Chinese hamster ovary cell line requiring ethanolamine or phosphatidylserine for growth and exhibiting defective phosphatidylserine synthase activity. Journal of Biological Chemistry. 261 1002-1008.

Vuilleumier, J. P. K. (1993). Fluorimetric assay of vitamin C in biological materials using a centrifugal analyser with fluorescence attachment. Journal of Micronutrient Analysis. 5 25-34.

Whipp, B. J., Ward, S. A., Lamarra, N., Davis, J. A. and Wasserman, K. (1982). Parameters of ventilatory and gas exchange dynamics during exercise. Journal of Applied Physiology. 52 1506-1513.

Yamatoya, H., Sakai, M. and Kudo, S. (2000). The effects of soybean transphosphatidylated phosphatidylserine on cholinergic synaptic functions of mice. Japanese Journal of Pharmacology. 84 93-96. 


\title{
Application for Ethical Approval \\ Department of Psychology, University of Wales Swansea
}

\section{Research Project in Collaboration with the Department of Sports Science, University of Wales Swansea}

\begin{abstract}
All Project students should complete this form in consultation with their project supervisor. If your supervisor feels that the project involves any potentially controversial procedures (i.e. they feel that the ethical issues raised need to be considered by the Department Ethics Committee) then the form should be placed in the tray in the Resources Centre for consideration by the Departmental Ethics Committee. Further advice will then be given to you by the Ethics Committee via your supervisor. The completed form should be bound along with your project report.
\end{abstract}

Project Title: The effects of chronic phosphatidylserine (PS) supplementation on recovery following intermittent exercise.

Principal investigator/Supervisor: Mike Kingsley; MSc (Lough), BPhEd (Otago), PGCE

Students Name: Daniel Wadsworth; BSc, (Wales)

Collaborator: Professor David Benton; $\mathrm{PhD}$

\section{Brief description of the purpose and methods of the project:}

Purpose: Phosphatidylserine (PS) has been shown to reduce blood levels of cortisol in response to resistance training (Fahey and Pearl, 1998) and physical exercise (Monteleone et al., 1992). The elevation of cortisol levels in response to exercise stress leads to the accelerated catabolism of amino acids. As such, elevated cortisol levels can be viewed as a possible precursor to muscle damage. Subsequently, suppression of cortisol levels could theoretically prevent muscle damage. Supplementation with PS may therefore enhance recovery following exercise. Burke (2000) supports the possible benefits on recovery of PS 
supplementation, suggesting that it may minimize muscle fibre damage caused by muscular stress.

Aims: To investigate the effects of chronic phosphatidylserine (PS) supplementation, on heart rate and cortisol levels, during and immediately following intermittent exercise. To investigate the effects of chronic phosphatidylserine supplementation (PS) supplementation on muscle soreness/damage following intermittent exercise.

No. of participants: $16-20$.

How and where are the participants to be recruited: Undergraduate and postgraduate male student volunteers will be recruited.

Experimental procedure (brief details only): Preliminary testing of subjects will include an estimation of maximal oxygen uptake, by multi-stage fitness test. Subjects will then complete a familiarization trial of a prolonged intermittent exercise protocol, designed to replicate the physiological demands of soccer match play. Subsequently, subjects will complete two further exercise trials, once with no supplement (control) (T1) and once with either a PS supplement or a glucose placebo (T2). Chronic supplementation of $900 \mathrm{mg} /$ day of PS or a weight matched glucose placebo (T2) will take place for 10 days prior to T2. Subjects will be randomly allocated to supplementation group using a double blind procedure. A minimum of 1 week will separate the familiarization and T1 trials, with $\mathrm{T} 2$ occuring approximately 2-4 weeks later.

During T1 and T2, physiological measurement procedures will consist of heart rate monitoring, 2 venous blood samples ( $<30 \mathrm{ml}$ per sample), and sprint/endurance performance assessment. Subjects will be required to return for tests 1 and 2 days after exercise to assess recovery, when further blood samples ( $<30 \mathrm{ml}$ per sample) will be collected in order to assess their recovery (one sample per day), and muscle soreness assessed. Blood samples will then be analysed for markers of muscle damage, oxidative stress and cortisol concentrations.

Details of any payment given: None 


\section{Consent and Debriefing}

Have you prepared a consent form for participants? YES (attached)

Have you prepared an information sheet to debrief participants? YES (attached)

You must attach the consent form and information sheet before handing this form to your supervisor. You must also attach a copy of any questionnaire(s) that you intend using.

Environmental validity questionnaire

Perceived muscle soreness questionnaire

\section{Ethical Considerations}

Please read the following declarations carefully and detail below any ways in which your project deviates from them. Then sign where indicated.

1. I have ensured that there will be no active deception of participants.

2. I have ensured that no data will be personally identifiable.

3. I have ensured that no participant should suffer any undue physical or psychological discomfort

4. I certify that there will be no administration of potentially harmful drugs, medicines or foodstuffs. *

5. I will obtain written permission from an appropriate authority before recruiting members of any outside institution as participants.

6. I certify that the participants will not experience any potentially unpleasant stimulation or deprivation?

7. I certify that any ethical considerations raised by this proposal have been discussed in detail with my supervisor.

8. I certify that the above statements are true with the following exception(s):

* Phosphatidylserine is a naturally occurring phospholipid predominantly found in the brain cell membranes but also found in other structures (RCC, 1996). It is considered a safe food supplement with no known side effects. The dosage given for this study will be $300 \mathrm{mg} /$ day in three $100 \mathrm{mg}$ tablets. This dose will be taken for 30 days.

Registration and consulting company. (1996) Expert report on the safety of Phophatidylserine PS 50 as produced from soya lecithin by enzymatic transesterification. RCC project 629910.

Signature:

Date: 
In the supervisor's opinion, this project (tick one only):

Does not raise any significant issues.

Raises some-ethical issues, but I consider that appropriate-steps and precations have been taken and 1 have approved the proposal and acept the responsibility for having done so.

Raises ethical issues that need to be considered by the Departmental Ethics Committee.

Raises ethical issues such that it should not be allowed to proced in its current form.

Supervisor's signature: Date:

(For Ethics Committee use only)

The ethical issues raised by this project have been considered by members of the Departmental Ethics Committee who made the following comments:

Please ensure that you take account of these comments and prepare a revised submission that should be shown to your supervisor/ resubmitted to the Department Ethics Committee (delete as appropriate).

Signed:

Date:

(Chair, Departmental Ethics Committee) 
PRIFYSGOL CYMRU ABERTAWE

Yr Adran Seicoleg

Ysgol Gwyddorau Dynol

Parc Singleton, Abertawe, SA2 8PP
UNIVERSITY OF WALES SWANSEA

Department of Psychology

School of Human Sciences

Singleton Park, Swansea, SA2 8PP

Mike Kingsley

Sports Science

$21 / 3 / 2002$

The effects of phosphatidylserine supplementation on recovery following prolonged intermittent exercise.

Dear Mike,

The departmental ethics committee has reviewed the above study and have agreed that it raises no substantive ethical issues, provided that the information obtained is kept confidential and that no personally identifiable information is entered onto computer.

With these provisions we are happy for you to proceed with your study.

Yours sincerely,

\section{X.Bstem}

Kate Bullen, Ph.D.

On behalf of the ethics committee

Pennaeth yr Adran - Profecicor Philip JJ. Corr - Head of Department Tel 01792295081 Fax 01792295679 Email psychology@swansea.ac.uk

Pennaeth vr Yggol - Professor Phil Reed . Head of School Tel 01792602047 Fax 01792295679 Email p.reed@swansea.ac.uk 


\section{UNIVERSITY OF WALES SWANSEA \\ DEPARTMENT OF SPORTS SCIENCE \\ DEPARTMENTAL ETHICS ADVISORY COMMITTEE}

\section{APPLICATION FOR ETHICAL COMMITTEE APPROVAL OF A RESEARCH PROJECT}

In accordance with Departmental Safety Policy, all research undertaken in the department must be approved by the Departmental Ethics Advisory Committee prior to data collection. Applications for approval should be typewritten on this form using the template available in the Public Folders. The researcher(s) should complete the form in consultation with the project supervisor. Where appropriate, the application must include the following appendices:

(A) subject information sheet;

(B) subject consent form;

(C) subject health questionnaire.

After completing sections 1-12 of the form, seven copies of the form should be handed into the Department Administrator who will submit the application for consideration by the Departmental Ethics Advisory Committee. The applicant(s) will be informed of the decision of the Committee in due course.

\section{DRAFT TITLE OF PROJECT}

Effects of Phosphatidylserine Supplementation on Muscle Damage, mental tasks and stress following Downhill Running

\section{NAMES AND STATUS OF RESEARCH TEAM}

Mr Mike Kingsley - Supervisor

Richard Elms - BSc Sports Science $3^{\text {rd }}$ year student

David Maycock - BSc Sports Science $3^{\text {rd }}$ year student

Gareth Matthews - BSc Sports Science $3^{\text {rd }}$ year student

Nick Hodgson $\quad-$ BSc Sports Science $3^{\text {rd }}$ year student

\section{RATIONALE}

Byrnes et al. (1985) suggested that downhill running is considered to induce delayed-onset muscle soreness (DOMS) due to bias towards eccentric contractions, with soreness peaking between 48 to 72 hours post exercise. Fahey et al. (1998) suggests that soreness is due to two different causes of damage to the muscle fibres: muscular damage, which is instant, caused by excessive force, and secondary cellular damage caused by elevation of proteases and phosopholipids due to physical stress on the body. An elevation in Cortisol is also achieved, which Fahey et al. (1998) suggested increased protein breakdown, and decreases protein synthesis, which may lead to the deterioration of muscle tissue. Studies based on the effects of PS supplementation, have mainly focused on the hypothalamopituitary-adrenal axis, and not the possible effects on DOMS. The studies by Monteleone et al. (1990) and Monteleone et al. (1992) concluded that PS supplementation did "blunt" the elevation of Cortisol and its controlling hormone adrenocorticotrophic hormone (ACTH). In theory, PS supplementation may decrease serum creatine kinase (CK) levels due to a reduction in fibre trauma, which may lead to a reduction symptoms of DOMS by decreasing muscle damage caused by the secondary cellular breakdown of protein by elevated levels of cortisol after exercise.

According to Gullick and Kimura (1996), muscle soreness can either be 'acute' or have a 'delayed onset' dependant on when muscle soreness occurs. Acute muscle soreness tends to transpire during the latter stages of exercise whereby, delayed onset muscle soreness (DOMS) is seen to peak 24-48 hours after exercise (Gullick and Kimura, 1996; Maughan et al., 1989). DOMS has been seen to have a negative effect on athletic performance, including tenderness to palpation and a decreased flexibility. The effect of repeated exercise and familiarisation bouts during experiments has been thought to diminish the muscle fibre injury resulting from eccentric exercise, which is the source of DOMS (Armstrong, 1986). The symptoms of DOMS have been seen to be markedly lower following 
general or task specific training (Byrnes et al., 1985). There has not been an experiment that looks directly at the effect of a familiarisation eccentric exercise bout on the symptoms of DOMS when compared to a non- familiarised bout. Previous studies have supported the hypothesis that a familiarisation bout of specific exercise causes a marked decrease in DOMS symptoms for up to three weeks (Byrnes et al., 1985).

The effects of physical exercise on mental function has been widely studied by psychologists; however, results have produced contradictory findings leading researchers identifying several methodological factors to control in studies including the nature of the psychological task, intensity and duration of physical exercise (Brisswalter, et al., 2002). Researchers have produced consistent results showing physical exercise enhances cognitive performance mainly through the use of decisional tasks. However, this is subject to the type, intensity and duration of the exercise. Brisswalter et al. (2001) found that an improvement in cognitive performance was observed with long-term aerobic exercise. Anaerobic exercise has also been found to increase cognitive performance with Davey (1973) finding that anaerobic exercise demonstrated facilitation in the subject's cognitive performance. Phosphatidylserine has been widely used as a memory aid through the treatment of patients with cognitive dysfunctions such as Alzheimers with some success (Crooks et al., 1992) and therefore the link has been made to use the supplement for reducing stress. Benton et al. (2001) found PS supplementation decreased feelings of being stressed after performing a demanding mental arithmetic task. However, nothing has been done on the effects that PS has on exercise stress of cognitive performance. Task complexity must also be taken into account when looking into the effects of cognitive performance during physical exercise, with the nature of the task interpret ting the allocation of attention capacity to the physical or mental task (Abernethy, 1988).

Physical exercise significantly increases blood lactate concentration. It is thought that PS may influence the response of the body to stress. This is thought to be due to exogenous PS affects the number and affinity of membrane receptor site (Stockert et al., 1989; De Robertis et al., 1989). This influences cell membrane transduction mechanisms such as specific protein kinase activation (Canonico and Scapagnini, 1989). Information transduction systems are important in cell to cell communication and therefore crucial in homeostatic adaptation to changing stimuli, such as stress. Thus PS may alter the bodies response to stress (Monteleone et al. 1991) by altering functional events in the cell Appendices Xl membrane. During physical exercise the hypothalamo-pituitary-adrenal (HP A) axis is stimulated. PS partly counteracts the activation of HPA. This suggests that Phosphatidylserine has an acute effect on the stress-induced release of the adrenocorticotrophic (ACTH) hormone and cortisol (Monteleone et al.., 1990), and therefore may alternate the physiological response to exercise.

\footnotetext{
4. REFERENCES

1. Abernethy, B. (1988). Dual task methodology and motor skills research: some applications and methodological constraints. Journal of Human Movement Studies, 14, 101-32

2. Armstrong, R.B. (1986). Muscle Damage and Endurance Events. Journal of Sports Medicine. 3, 370-381

3. Benton, D., Donohoe, R.T., Sillance, B., Nabb, S. (2001). The influence of phosphatidylserine supplementation on mood and heart rate when faced with an acute stressor. Nutritional Neuroscience, 4, 169-178.

3. Byrnes, W.C., Clarkson, P;M., White, J.S., Hsieh, S.S., Frykman, P.N., \& Maughan, R.J. (1985). Delayed onset muscle soreness following repeated bouts of downhill running. Journal of Applied Physioliolgy 59, 710-715

4. Brisswalter, J., Collardeau, M., and Rene, A. (2002). Effects of acute physical exercise characteristics on cognitive performance. Sports Medicine, 32, 555-66.

5. Collardeau, M., Brisswalter, J., and Audiffren, M. (2001). Effects of a prolonged run on simple reaction time of well trained runners. Perceptual and Motor Skills, 93, 679-689.

6. Davey, C.P. (1973). Physical exertion and mental performance, Ergonomics, 16, 595-599.

6. Fahey, T.D. and Pearl, M.S. (1998). The Hormonal and Perceptive Effects of Phosphatidylserine Administration During two Weeks of Resistive Exercise-Induced Overtraining. Biology of Sport. 15, 135-144.

7. Gulick, D.T. and Kimura, I.F. (1996) Delayed Onset Muscle Soreness: What is it and how do we treat it? Journal of Sports Rehabilitation. 5, 234-236.
} 
8. Maughan, R.J., Donnelly, A.E., Gleeson, M., Whiting, P.H., Walker,K.A. and Clough, P.J. (1989). Delayed-onset muscle damage and lipid peroxidation in man after a downhill run. Muscle and Nerve. 12, 332-336.

9. Monteleone, P., Beinat, L., Tanzillo, C., Maj, M. and Kemali, D. (1990). Effects of Phosphatidylserine on the Neuroendocrine Response to Physical Stress in Humans. Neuroendocrinology. 52 , 243-248.

10. Monteleone, P., Beinat, L., Maj, M., Natale, M., and Kemali, D. (1992). Blunting by Chronic Phosphatidylserine Administration of the stress-induced activation of the Hypothalamo-Pituitary-Adrenal axis in healthy men. European Journal of Clinical Pharmacology. 41, 385-388.

11. Tomporowski P.D., and Ellis, N.R. (1986). Effects on Cognitive Processes: A Review. Psychological Bulletin, 99, 338-336.

\section{AIMS and OBJECTIVES}

The aim of the study is to determine the effect of chronic PS supplementation on Physiological stress, oxidative stress and the development of DOMS on 8, 19-24 year old male subjects.

The specific objectives are:

1. To determine the effect of a familiarisation bout development of DOMS.

2. To investigate the effect of PS on markers of oxidative stress and inflammation that follow downhill running.

3. Assess the effects of PS supplementation on muscle damage and self reported DOMS after downhill running. 4. Assess the effects of PS supplementation on the performance of mental tasks following downhill running.

\section{METHODOLOGY}

\subsection{Study Design}

8 male physically fit subjects aged from 19-24 will be asked to complete the study. All prior to participation subjects will be required to complete an AHA ACSM pre-participation screening questionnaire. Subjects will be required to take part in a progressive maximal exercise test, and five thirty minute downhill runs each followed by a cognitive task, and 24 and 48 hours after each run flexibility and blood samples will be taken. Subjects will complete a preliminary testing session; a familiarization session and four main trials over duration of 10 weeks. 10 days prior to the second main trial the subjects will receive either a PS supplement or Placebo.

\subsection{Experimental Procedures}

Preliminary Testing: Subjects will complete a progressive maximal exercise test in order to determine the exercise intensity for the downhill exercise trials.

Familiarisation and main trials: Subjects will complete a 5 minute warm up jog on the treadmill at there own selected pace. This will be followed by a 30 minute downhill run at a gradient of -10 percent and a work rate equivalent to approximately $70 \%$ of their maximum heart rate. Measurements of DOMS (perceived muscle soreness), range of motion and venous blood samples $(<30 \mathrm{ml}$ per sample) will be taken at the following time points during each trial (before exercise, after exercise, 24 hours after exercise and 48 hours after exercise).

Supplementation: The subjects will be required to take an oral supplement containing either approximately $800 \mathrm{mg} /$ day for 10 days prior to and for 2 days after main trials 2 and 4 . Subjects will receive either PS or glucose supplements in a balanced cross-over design.

\subsection{Data Analysis Techniques}

Statistical analysis of the data will be completed using statistical packages such as Excel and SPSS.

\subsection{Storage and Disposal of Data and Samples}

Data will be stored electronically; however, access to the data will be restricted to the research team. Any published information will not include any information that would potentially reveal the identification of subjects. Blood samples will be stored prior to analysis and then disposed of using standard biological procedures. 


\section{LOCATION OF THE PREMISES WHERE THE RESEARCH WILL BE CONDUCTED.}

Exercise Physiology Labs, Vivian Tower, University of Wales Swansea

\section{SUBJECT RISKS AND DISCOMFORTS}

Subjects will be completing 5 downhill runs at approximately $70 \%$ of their maximum heart rate and as a result they are expected to exhibit some degree of muscle soreness; however, all subjects will be familiar with DOMS. All the subjects will be monitored immediately post exercise and any risk due to blood testing will be minimized through strict adherence to standard procedures as specified in the department safety policy. In addition, prior to testing health screening will be fulfilled using the AHA/ACSM pre-participation screening questionnaire (Appendix C). Subjects will be required to take an oral supplement containing PS; PS is a naturally occurring phospholipid that is predominantly found in the cell membranes and is considered safe to use and is currently marketed as a memory aid.

\section{INFORMATION SHEET AND INFORMED CONSENT}

The submission should be specific about the type of consent that will be sought:

Have you included a Subject Information Sheet for the participants of the study? YES (Appendix A)

Have you included a Subject Consent Form for the participants of the study? YES (Appendix B)

If written consent will not be obtained, explain why

\section{COMPUTERS}

Are computers to be used to store data? YES

If so, is the data registered under the Data Protection Act? YES

NB : For UWS students, the answer to this question is YES, but the question has been included in order to stress the importance of adherence to the Data Protection Act in research activity

\section{STUDENT DECLARATION}

Please read the following declarations carefully and provide details below of any ways in which your project deviates from them. Having done this, each student listed in section 2 is required to sign where indicated.

1. I have ensured that there will be no active deception of participants.

2. I have ensured that no data will be personally identifiable.

3. I have ensured that no participant should suffer any undue physical or psychological discomfort

4. I certify that there will be no administration of potentially harmful drugs, medicines or foodstuffs.

5. I will obtain written permission from an appropriate authority before recruiting members of any outsideinstitution as participants.

6. I certify that the participants will not experience any potentially unpleasant stimulation or deprivation.

7. I certify that any ethical considerations raised by this proposal have been discussed in detail with my supervisor.

8. I certify that the above statements are true with the following exception(s):

Student signature: Date:

Student signature: Date:

Student signature: Date:

Student signature: Date: 


\section{SUPERVISOR'S DECLARATION}

In the supervisor's opinion, this project (delete those that do not apply):

- Does raise-any significant issues.

- Raises some ethical issues, but I consider that appropriate steps and precautions have been taken and I have approved the proposal.

- Raises ethical issues that need to be considered by the Deparmental Ethics Committee.

- Raises ethical isstes such that it should not be allowed to proced in its current form.

Supervisor's signature:

Date:

\section{ETHICS COMMITTEE DECISION (COMMITTEE USE ONLY) \\ ETHICAL APPROVAL: GRANTED REJECTED (delete as appropriate)}

The ethical issues raised by this project have been considered by members of the Departmental Ethical Approval Committee who made the following comments:

Please ensure that you take account of these comments and prepare a revised submission that should be shown to your supervisor/ resubmitted to the Department Ethical Approval Committee (delete as appropriate).

Signed:

Date:

(Chair, Departmental Ethics Advisory Committee) 


\section{PRIFYSGOL CYMRU ABERTAWE UNIVERSITY OF WALES SWANSEA \\ Department of Sports Sclence \\ Departmental Ethical Advisory Committee}

From : $\quad$ Dr M J Lewis, Chairman, DEAC

To : $\quad$ Mr M Kingsley, Mr R Elms, Mr G Matthews and Mr N Hodgson

Subject : $\quad$ Application for Ethics Committee Approval

Date : $\quad 25 / 11 / 03$

Your application (submitted 29/10/03) for ethical approval for :

Effects of phosphotidylserine supplementation on muscle damage, mental tasks and stress following downill running.

was considered by the Departmental Ethics Advisory Committee members on 5/11/03. DEAC Committee approval subject to conditions and resubmission was given.

Following resubmission of the application, members of the DEAC agree that the study raises no substantive ethical issues. Therefore, Chair's approval granted 20/11/03.

MJL. 


\title{
Application for Ethical Approval \\ Department of Psychology, University of Wales Swansea
}

\section{Research Project in Collaboration with the . Department of Sports Science, University of Wales Swansea}

\begin{abstract}
All Project students should complete this form in consultation with their project supervisor. If your supervisor feels that the project involves any potentially controversial procedures (i.e. they feel that the ethical issues raised need to be considered by the Department Ethics Committee) then the form should be placed in the tray in the Resources Centre for consideration by the Departmental Ethics Committee. Further advice will then be given to you by the Ethics Committee via your supervisor. The completed form should be bound along with your project report.
\end{abstract}

Project Title: Chronic phosphatidylserine (PS) supplementation and its effects on recovery following exercise stress.

Principal investigator/Supervisor: Mike Kingsley; MSc (Lough), BPhEd (Otago), PGCE

Students Name: Mark Miller; BSc, (Wales)

Collaborator: Professor David Benton; $\mathrm{PhD}$

Brief description of the purpose and methods of the project:

Aims \& purpose: To investigate the effects of chronic PS supplementation and on recovery following intermittent running.

No. of participants: Approximately twenty-four.

How and where are the participants to be recruited: Undergraduate and postgraduate student volunteers will be recruited. 
Experimental procedure (brief details only): Chronic supplementation of PS ( $800 \mathrm{mg} / \mathrm{day}$ for 10 days) will be allocated using a placebo controlled double blind procedure. Preliminary testing will include anthropometric measurement and a progressive maximal exercise test on a cycle ergometer. Subjects will then undergo a familiarisation trail followed by 2 trials (pre and post supplementation). The exercise administration will be in the form of a staged intermittent cycling exercise with passive recovery periods. Measurement procedures will consist of heart rate monitoring, venous blood samples, respiratory gas analysis and other non-invasive procedures. A full description of the study design is attached.

Details of any payment given: None

\section{Consent and Debriefing}

Have you prepared a consent form for participants? YES (attached)

Have you prepared an information sheet to debrief participants? YES (attached)

You must attach the consent form and information sheet before handing this form to your supervisor. You must also attach a copy of any questionnaire(s) that you intend using.

\section{Ethical Considerations}

Please read the following declarations carefully and detail below any ways in which your project deviates from them. Then sign where indicated.

1. I have ensured that there will be no active deception of participants.

2. I have ensured that no data will be personally identifiable.

3. I have ensured that no participant should suffer any undue physical or psychological discomfort

4. I certify that there will be no administration of potentially harmful drugs, medicines or foodstuffs. *

5. I will obtain written permission from an appropriate authority before recruiting members of any outside institution as participants.

6. I certify that the participants will not experience any potentially unpleasant stimulation or deprivation?

7. I certify that any ethical considerations raised by this proposal have been discussed in detail with my supervisor.

8. I certify that the above statements are true with the following exception(s): 
* Phosphatidylserine is a naturally occurring phospholipid predominantly found in the brain cell membranes but also found in other structures (RCC, 1996). It is considered a safe food supplement with no known side effects. The dosage given for this study will be $300 \mathrm{mg} / \mathrm{day}$ in three $100 \mathrm{mg}$ tablets. This dose will be taken for 30 days.

Registration and consulting company. (1996) Expert report on the safety of Phophatidylserine PS 50 as produced from soya lecithin by enzymatic transesterification. RCC project 629910.

Signature: Date:

In the supervisor's opinion, this project (tick one only):

Does raise any significant issues.

Raises some ethical issues, but I consider that appropriate-steps and precautions have been taken and $I$ have approved the proposal and a the respensibility for having doneso. Raises ethical issues that need to be considered by the Departmental Ethics Committee. Raises ethical issues-such that it should not be allowed to proced in its current form.

Supervisor's signature: Date:

(For Ethics Committee use only)

The ethical issues raised by this project have been considered by members of the Departmental Ethics Committee who made the following comments:

Please ensure that you take account of these comments and prepare a revised submission that should be shown to your supervisor/ resubmitted to the Department Ethics Committee (delete as appropriate).

Signed:

Date: $21 / 03 / 2002$

(Chair, Departmental Ethics Committee) 
PSYCHOLOGY

DEPPATCAOLOCY

DEPARTMENT

EHHCS Coninitias

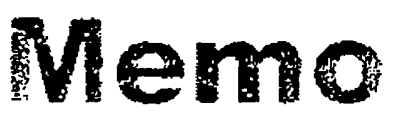
To: Professor David Beaton

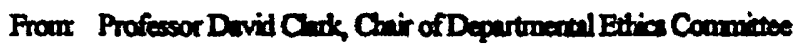
Dete: $19^{6}$ Merch, 2003

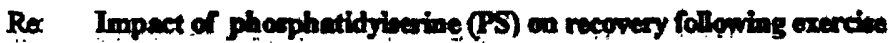

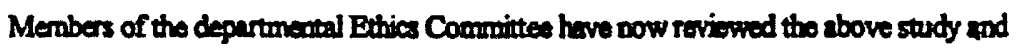

agree that it ribes no substintive chical ivares, provided the information obtained froen the

questionmires is kept absolutely confidentil and that wo personelly identifabb information is

entered oo compuner. You may therefore proceed with your stuty.

- Puge 2 


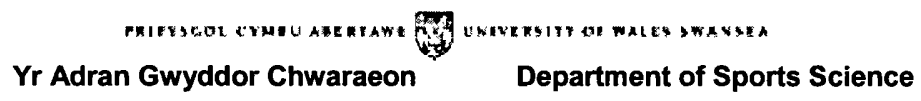

\author{
Contact Details: \\ Mr Mike Kingsley \\ Lecturer in Sports Science \\ Department of Sports Science \\ Vivian Tower \\ University of Wales Swansea, SA28PP \\ Tel: 01792295086
}

\begin{abstract}
1. Study title
The effects of chronic phosphatidylserine (PS) supplementation on recovery following prolonged intermittent exercise.
\end{abstract}

\title{
2. Invitation paragraph
}

You are being asked to volunteer for this research study, which is being conducted for research. It is important that before you decide to take part you know what it will involve. If you do not understand anything please feel free to ask. Consumers for ethics in research (CERES) publish a leaflet entitled "medical research and you". This leaflet gives more information about medical research and looks at some questions you may wish to ask. A copy may be obtained from CERES, PO box 1365 London N16 OBW. Thank you for reading this.

3. What is the purpose of this study?

This study aims to investigate the effect of chronic supplementation of PS on recovery. The shortterm effects (immediately after exercise) of supplementation on heart rate, oxidative stress and cortisol levels will be investigated, as will long-term (up to 48 hours post exercise) effects on muscle soreness, damage and oxidative stress.

\section{Why have I been chosen?}

You have been chosen as a subject for this study as you are an active male, aged 18-25 years old. Your participation in this study is entirely voluntary, and you should be aware that you have the right to withdraw at any time without providing a reason.

\section{What will happen to me if I take part?}

Preliminary testing will consist of a multi-stage fitness test (the "beep test") in order to assess your fitness. After this, you will complete an exercise protocol designed to simulate the activity levels and demands of a soccer match. The protocol consists of 90 minutes of walking, jogging, cruising and sprinting, and also includes frequent rest periods (1 minute breaks) and a 15 minute "half-time".

You will need to complete this protocol on 3 separate occasions, the first trial will familiarize you with the protocol. You will then complete two further trials, once with no supplement (control) (T1) and once with either a PS supplement or a glucose placebo (T2). You will be supplemented with either $900 \mathrm{mg}^{-\mathrm{day}^{-1}}$ of PS or a glucose placebo for 10 days prior to T2.

During T1 and T2, physiological measurement procedures will consist of heart rate monitoring and sprint/endurance performance assessment before and after T1 and T2.

Blood will be taken intravenously, $(<30 \mathrm{ml}$ per sample). You will be required to return for tests 1 and 2 days after exercise to assess your recovery, when further blood samples will be collected (one each day), and your soreness will be assessed by questionnaire. 


\section{What are the possible disadvantages of taking part?}

As the study aims to assess the effects of PS supplementation with regards to muscle soreness, subjects are expected to feel some degree of discomfort on the days following exercise.

Blood samples are to be taken intravenously by a trained individual, and whilst this is not a painful process, subjects must be comfortable with such collection methods. Even though the volume of blood taken is small ( $<30 \mathrm{ml}$ per sample) there exists the possibility that during or immediately following this procedure subjects may feel light headed and faint. There is an extremely small risk that this procedure could result in an air or plastic embolism, but good practice minimises this risk. In addition, every effort will be made to minimise the risks of contaminating the wound by using sterile disposable equipment and standardised procedures for the collection and disposal of biohazard wastes.

\section{What are the possible benefits of taking part?}

Taking part in this study will allow you to assess your fitness at the time of the study, and also follow your progress throughout the duration of the study. It has been suggested that PS may improve confidence and composure during exercise, which is clearly of benefit. In addition, PS may enhance perceived soreness following exercise. As such, PS may enhance recovery and therefore may have possible benefits on future exercise bouts.

\section{Will my taking part in the study be kept confidential?}

Whilst the study will be available to all within the University of Wales Swansea, all results from this study will remain anonymous. 
Version 1.0

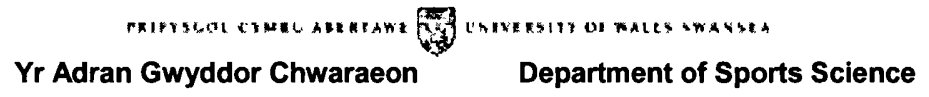

Date: $15^{\text {th }}$ October 2003

Mr Mike Kingsley

Lecturer in Sports Science

Department of Sports Science

Vivian Tower

University of Wales Swansea, SA28PP

Tel: 01792295086

\section{Study title}

Effects of Phosphatidylserine supplementation on muscle damage, mental tasks and stress from downhill running.

\section{Invitation paragraph}

You are invited to take part in this study to help with research. You are more than welcome to ask questions at any time and you are able to withdraw from any part of the study at any time, without being asked to give a reason.

\section{What is the purpose of this study?}

The purpose of this study is to investigate the effects of phosphatidylserine (PS) supplementation on muscle damage, mental tasks and stress levels during and after five bouts of downhill running. In particular, the effects on markers of muscle damage, oxidative stress, inflammation and severity of delayed onset of muscle soreness (DOMS). This study will provide an idea into the possible benefits of PS (if any) being supplemented to athletes for use with eccentric muscle activities. The study also aims to investigate the effect of familiarisation bout of exercise on flexibility and DOMS.

\section{Why have I been chosen?}

All subjects are volunteers from the University of Wales Swansea (UWS) and consists of undergraduates males aged between 19-24 years old. Approximately 16 subjects will take part.

\section{What will happen to me if I take part?}

If you choose to participate in this study then you will be required to carry out physical tests used to assess your fitness. You will be required to visit the exercise physiology laboratory during five further exercise trials, each visit will last approximately 1 hour. During these trials you will be asked to complete $30 \mathrm{~min}$ of downhill treadmill running, which is used to promote DOMS in the legs. Flexibility tests, mental task testing and venous blood samples ( $<30 \mathrm{ml}$ per sample) will be taken preexercise, post-exercise at 24 hours and 48 hours after exercise. Prior to the third and fifth treadmill run you will be required to undertake a ten-day oral supplementation period, during which time you will consume either $800 \mathrm{mg} \cdot \mathrm{day}^{-1}$ of phosphatidylserine (PS) or a placebo, in a cross-over design.

\section{What are the possible disadvantages of taking part?}

The risks associated with exercise are very small. However, this will be further minimised by the health screening you will complete prior to undertaking exercise. There is an extremely small risk that venepuncture could result in an air or plastic embolism, but good practice minimises this risk. The samples will be taken by trained staff members.

\section{What are the possible benefits of taking part?}

Possible benefits for receiving the PS supplement include a reduction in DOMS and improved recovery following exercise.

\section{Will my taking part in the study be kept confidential?}

All information collected about you will be kept strictly confidential. Any information that is distributed by the Department of Sports Science will only be identifiable by number and not name. 


\title{
Contact Details: \\ Mr Mike Kingsley \\ Lecturer in Sports Science \\ Department of Sports Science \\ Vivian Tower \\ University of Wales Swansea, SA28PP \\ Tel: 01792295086
}

\author{
1. Study title \\ Chronic phosphatidylserine (PS) supplementation and its effects on recovery following exercise \\ stress.
}

\section{Invitation paragraph}

You are being asked to volunteer for this research study. It is important that before you decide to take part you know what it will involve. Take time to read this information sheet and discuss, if you feel you need to with friends, relatives or even you're G.P. If you do not understand anything please feel free to ask. Consumers for ethics in research (CERES) publish a leaflet entitled "medical research and you". This leaflet gives more information about medical research and looks at some questions you may wish to ask. A copy may be obtained from CERES, PO box 1365 London N16 OBW. Thank you for reading this.

\section{What is the purpose of this study?}

The study aims to investigate the effect of chronic supplementation of PS on recovery following exercise. It has been suggested that supplementation may increase the rate of recovery following exercise.

\section{Why have I been chosen?}

All subjects are volunteers from the University of Wales Swansea (UWS). They will consist of undergraduates and graduates. Approximately 24 subjects will take part.

\section{Do I have to take part?}

Taking part is entirely voluntary. If you do decide to take part you will be given this information sheet to keep and will be asked to sign a consent form. Even if you decide to take part you are free to withdraw at any time without a reason. This will not affect the treatment you receive.

\section{What will happen to me if I take part?}

If you decide to take part in this study you will be required to visit the exercise physiology laboratory four times, each visit will last approximately 1 hour. Your first three visits will take place over the space of one to two weeks. During these visits you will complete preliminary tests, a familiarisation trial and the pre-supplement main trial. You will then be required to undertake a ten-day oral supplementation period, during which time you will consume either $800 \mathrm{mg}^{- \text {day }^{-1}}$ of phosphatidylserine (PS) or a placebo. A placebo is a dummy treatment, which looks like the real thing but contains no active ingredients. You will be randomly assigned to either the PS or placebo group in such a way that neither yourself of the researcher will know which group you are in until the end of the study. Following the supplementation period you will complete the post-supplement main trial. 
You will be required to complete the following:

1) Preliminary testing: Following the completion of a health screening questionnaire your weight, height and body composition will be measured. Following this you will complete an exercise test at progressively increasing intensity until you are unable to continue exercising. Heart rate, perceived exertion and respiratory data will be monitored throughout. This assessment will be used to identify your maximal exercise capacity and calculate the intensities you will complete each main trial.

2) Familiarisation: You will undergo an intermittent exercise test on an exercise ergometer. The test will require you to complete five 6-min bouts of exercise separated by approximately 10-min of recovery. The work rate for the first exercise bout will start at a relatively low intensity and progress to a moderately hard intensity for the last exercise bout.

3) Main trials: During both main trials you will complete the same intermittent exercise test as completed during the familiarisation. Your heart rate and expired respiratory gas will be monitored throughout the trials. In addition, $10 \mathrm{ml}$ venous blood samples will be obtained by the means of an indwelling cannula (a thin, flexible plastic tube) or venepuncture (a small hollow needle) inserted in a forearm vein. Blood samples will be taken prior to exercise and after each recovery period.

4) Additional requirements: You will be required to consume oral supplementation (PS; 300 $\mathrm{mg} \cdot \mathrm{day}^{-1}$ or placebo) over a 30-day period and complete 3-day exercise and dietary records during the week prior to each main trial. You will be asked fast overnight before reporting to the laboratory.

\section{What is the supplement that is being tested?}

Phosphatidylserine are naturally occurring phospholipids predominantly found in cell membranes and other structures. It is considered safe to use and is currently on the market as a memory aid.

\section{What are the effects of taking part?}

There are no known side effects of the supplement, however, if you experience any adverse effects please contact us straight away.

\section{What are the possible disadvantages of taking part?}

The acute risks associated with exercise are very small. However, this will be further minimised by the health screening you will complete prior to undertaking exercise. There is an extremely small risk that cannulation could result in an air or plastic embolism, but good practice minimises this risk. The samples will be taken by trained staff members.

\section{What are the possible benefits of taking part?}

If you receive the PS supplement it could benefit your recovery from exercise. Preliminary studies have shown this might be the case.

\section{Will new information become available?}

New information can always come to light about research areas. If this happens while the study is taking place you will be informed and a course of action will be decided.

\section{What happens when the research study stops?}

The supplement is available on the open market if you wish to carry on taking it.

14. Will my taking part in the study be kept confidential?

All information collected about you will be kept strictly confidential. Any information that is distributed by the Department of Sports Science will only be identifiable by number and not name.

\section{What will happen to the results of the research study?}

The results of the study will be disseminated to the wider Sports Science and Psychology community. It is intended that this will be available late 2002 early 2003.

16. Who is organising the research?

The Department of Sports Science UWS is carrying out the research in collaboration with the Department of Psychology UWS. 
Yr Adran Gwyddor Chwaraeon

Department of Sports Science

AHA/ACSM Health/Fitness Facility Preparticipation Screening Questionnaire.

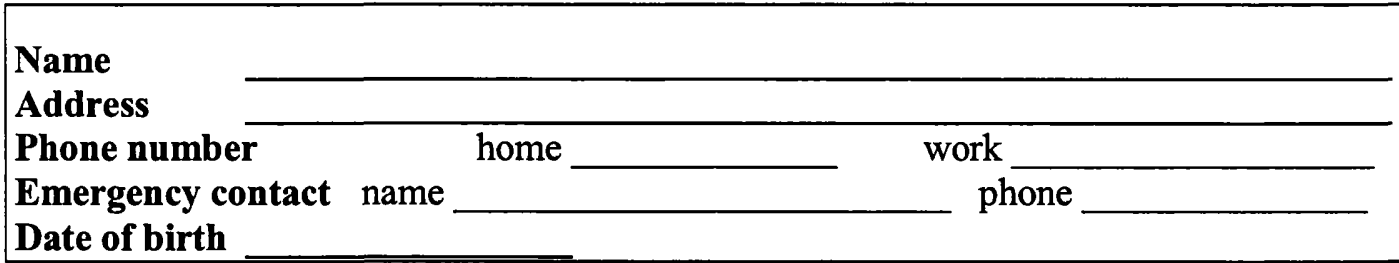

History Assess your health needs by marking all true statements.

You have had:

$\square$ a heart attack

$\square$ heart surgery

$\square$ cardiac catheterization

$\square$ coronary angioplasty (PTCA)

$\square$ pacemaker/implantable cardiac

defibrillator/rhythm disturbance

$\square$ heart valve disease

$\square$ heart failure

पheart transplantation

$\square$ congenital heart disease

\section{Symptoms and other health issues:}

$\square$ You experience chest discomfort with exertion.

$\square$ You experience unreasonable breathlessness.

$\square$ You experience dizziness, fainting, blackouts.

$\square$ You take heart medications.

$\square$ You take prescription medication(s).

$\square$ You have musculoskeletal problems.

$\square$ You have concerns about the safety of exercise.

$\square$ You are pregnant.

Cardiovascular risk factors

$\square$ You are a man older than 45 years.

$\square$ You are a woman older than 55 years or you have had a

hysterectomy or you are postmenopausal.

$\square$ You smoke.

$\square$ Your blood pressure is greater than $140 / 90$.

$\square$ You don't know your blood pressure.

$\square$ You take blood pressure medication.

$\square$ Your blood cholesterol level is $>240 \mathrm{mg} / \mathrm{dL}$.

$\square$ You don't know your cholesterol level.

$\square$ You have a blood relative who had a heart attack

before age 55 (father or brother) or age 65 (mother or sister).

If you marked any of the statements in this section, consult you a health provider before engaging in exercise. You may need to use a facility with a $\square$ You are diabetic or take medicine to control your blood sugar

$\square$ You are physically inactive (i.e., you get less than 30 minutes medically qualified staff.

of physical activity on at least 3 days per week).

$\square$ You are more than 20 pounds overweight.

$\square$ None of the above is true.

You should be able to exercise safely without consulting your healthcare provider in almost any facility that meets your exercise programme needs.

Declaration:
Please sign below to confirm that you have answered questions honestly and to the best of your ability.
Signature $\quad$ Date

AHA/ACSM indicates American Heart Association/American College of Sports Medicine. 


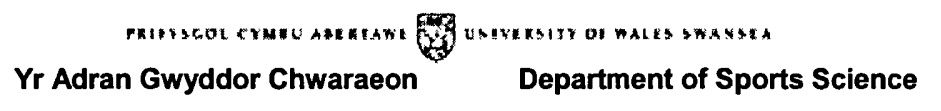

\section{Contact Details:}

Department of Sports Science

Vivian Tower

University of Wales Swansea, SA28PP

Tel: 01792295086

The effects of chronic phosphatidylserine (PS) supplementation on recovery following prolonged intermittent exercise.

\section{Please initial box}

1. I confirm that I have read and understood the information sheet dated ................... (version number study and have had the opportunity to ask questions. ) for the above

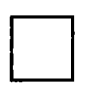

2. I understand that my participation is voluntary and that I am free to withdraw at any time, without giving any reason, without my medical care or legal rights being affected.

3. I understand that sections of any of data obtained may be looked at by responsible individuals from the University of Wales Swansea or from regulatory authorities where it is relevant to my taking part in research. I give permission for these individuals to have access to these records.

4. I agree to take part in the above study.

Name of Subject

Name of Person taking consent

Researcher
Date

Date

Date
Signature

Signature

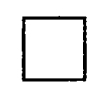




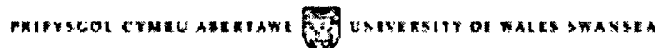 \\ Yr Adran Gwyddor Chwaraeon \\ Department of Sports Science
}

\title{
Contact Details:
}

Department of Sports Science

Vivian Tower

University of Wales Swansea, SA28PP

Tel: 01792295086

Effects of phosphatidylserine (PS) supplementation on muscle damage, mental tasks and stress following downhill running

\section{Please initial box}

1. I confirm that I have read and understood the information sheet dated .................. (version number .....................) for the above study and have had the opportunity to ask questions.

2. I understand that my participation is voluntary and that I am free to withdraw at any time, without giving any reason, without my medical care or legal rights being affected.

3. I understand that sections of any of data obtained may be looked at by responsible individuals from the University of Wales Swansea or from regulatory authorities where it is relevant to my taking part in research. I give permission for these individuals to have access to these records.

4. I agree to take part in the above study.

Name of Subject

Name of Person taking consent

Researcher
Date

Date

Date
Signature

Signature

Signature 


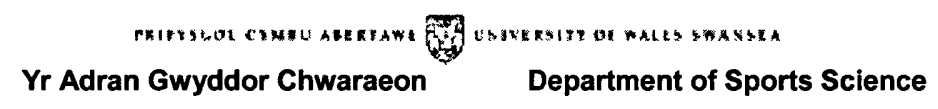

\section{Contact Details:}

Department of Sports Science

Vivian Tower

University of Wales Swansea, SA28PP

Tel: 01792295086

Chronic phosphatidylserine (PS) supplementation and its effects on recovery following exercise stress

\section{Please initial box}

1. I confirm that I have read and understood the information sheet dated ................... (version number ........................) for the above study and have had the opportunity to ask questions.

2. I understand that my participation is voluntary and that I am free to withdraw at any time, without giving any reason, without my medical care or legal rights being affected.

3. I understand that sections of any of data obtained may be looked at by responsible individuals from the University of Wales Swansea or from regulatory authorities where it is relevant to my taking part in research. I give permission for these individuals to have access to these records.

4. I agree to take part in the above study.

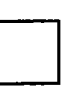


Appendix E: Perceived Muscle Soreness Scale

Instructions: Please use the following scale to indicate the extent to which each word below describes how sore your muscles feel at this moment in time.

\begin{tabular}{ll}
$\mathbf{0}$ & Not Sore \\
\hline 1 & \\
2 & Light soreness \\
\hline 3 & \\
\hline 4 & Somewhat sore \\
\hline 5 & \\
\hline 6 & Sore \\
\hline 7 & \\
\hline 8 & Very sore \\
\hline 9 & \\
\hline
\end{tabular}

10 Very, very sore 
Instructions: Please use the following scale to indicate the extent to which each word below describes how you feel at this moment in time. Record your responses by placing an $\mathbf{X}$ in the appropriate box.

$$
\begin{array}{ll}
0 & =\text { Do Not Feel [DNF] } \\
1 & =\text { Feel Slightly } \\
2 & =\text { Feel Moderately } \\
3 & =\text { Feel Strongly } \\
4 & =\text { Feel Very Strongly [FVS] }
\end{array}
$$

\begin{tabular}{|l|c|c|c|c|c|}
\multicolumn{1}{c|}{ DNF } & \multicolumn{1}{c|}{ FVS } \\
\cline { 2 - 6 } \multicolumn{1}{c|}{ 1. Refreshed } & & & & & \\
\hline 2. Calm & & & & & \\
\hline 3. Fatigued & & & & & \\
\hline 4. Enthusiastic & & & & & \\
\hline 5. Relaxed & & & & & \\
\hline 6. Energetic & & & & & \\
\hline 7. Happy & & & & & \\
\hline 8. Tired & & & & & \\
\hline 9. Revived & & & & & \\
\hline 10. Peaceful & & & & & \\
\hline 11. Worn-out & & & & & \\
\hline 12. Upbeat & & & & & \\
\hline
\end{tabular}

